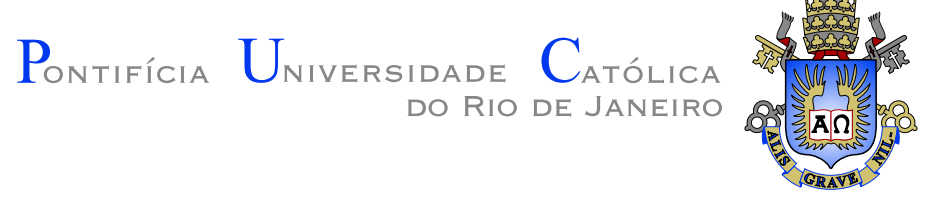

Thuener Armando da Silva

\title{
Optimization Under Uncertainty for Asset
}

\section{Allocation}

TESE DE DOUTORADO

Thesis presented to the Programa de Pós-Graduação em Informática of the Departamento de Informática da PUCRio as partial fulfillment of the requirements for the degree of Doutor.

Advisor : Prof. Marcus Vinicius Soledade Poggi de Aragão

Co-Advisor:

Prof. Davi Michel Valladão 


\section{Thuener Armando da Silva}

\section{Optimization Under Uncertainty for Asset \\ Allocation}

Thesis presented to the Programa de Pós-Graduação em Informática of the Departamento de Informática of Centro Técnico Científico da PUC-Rio, as partial fulfillment of the requirements for the degree of Doutor.

Prof. Marcus Vinicius Soledade Poggi de Aragão

Advisor

Departamento de Informática - PUC-Rio

Prof. Davi Michel Valladão

Co-Advisor

Departamento de Engenharia Industrial - PUC-Rio

Prof. Hélio Cortes Vieira Lopes

Departamento de Informática - PUC-Rio

Prof. Alexandre Street de Aguiar

Departamento de Engenharia Elétrica - PUC-Rio

Prof. Vitor Luiz de Matos

PLAN4

Prof. Geraldo Gil Veiga

RN Tecnologia

Prof. Bruno da Costa Flach

IBM

Prof. José Eugenio Leal

Coordinator of the Centro Técnico Científico da PUC-Rio

Rio de Janeiro, April 06, 2015 
All rights reserved.

\section{Thuener Armando da Silva}

Thuener Silva graduated as Bachelor in Computer Science at PUCRio in 2007. During graduate developed a project that used machine learning techniques for sentiment analysis with Raúl Renteria. In 2010, completed his Master in Computer Science in Optimization and Automatic Reasoning area. Developed the thesis about the portfolio selection applied to the Brazilian financial market entitled Experimental Study of Techniques for Portfolio Optimization with his advisor Eduardo Laber. During the Master received the scholarship from $\mathrm{CNPq}$ and maintained an excellent academic performance. His experience in Computer Science has an emphasis in Algorithms, Machine Learning, Information Retrieval, Portfolio Selection and Quantitative Methods.

Bibliographic Data

Silva, Thuener

Optimization Under Uncertainty for Asset Allocation / Thuener Armando da Silva; Advisor: Marcus Vinicius Soledade Poggi de Aragão; Co-advisor: Davi Michel Valladão. - 2015 .

99 f: il. (color.) ; $30 \mathrm{~cm}$

Tese (Doutorado em Informática) - Pontifícia Universidade Católica do Rio de Janeiro, Departamento de Informática, 2015.

Inclui bibliografia

1. Informática - Teses. 2. Seleção de Carteiras. 3. Alocação de Ativos em multi-estágio. 4. Análise de Investimentos. 5. Método de Apoio à Tomada de Decisão. 6. Black Litterman. 7. Programação Dinâmica Dual Estocástica. I. Poggi, Marcus Vinicius. II. Valladão, Davi Michel. III. Pontifícia Universidade Católica do Rio de Janeiro. Departamento de Informática. IV. Título. 


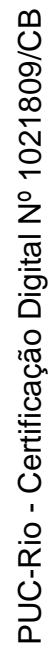

To my wife, my daughter and my family. 


\section{Acknowledgments}

I would like first to thank my wife Luana, for your unconditional support throughout this long journey. This work was only possible thanks to your help, patience and understanding. During all these years by your side your kindness and affection shown me the amazing person you are and how wonderful is our love, you make my life better every day.

Family is the most important thing in my life, thanks to my family for making me who I' am, especially to my parents, Fernando and Katia, my sisters and my brothers-in-law. Also, thank to my father-In-law, Gerson, for the guidance and revision on this work.

Thanks to my advisor Marcus Poggi for the support over these years, without your help this work would be impossible, you inspired me to be better every day. Also, thanks to my co-supervisor David Valladão, for your patience and dedication during this work, you believed in me even when I was very skeptical, it is a great pleasure to work with you. I also want to thank the professors Plácido Pinheiro, Alexandre Street for their involvement in this research.

Friends and colleagues make this difficult journey much easier, a special thanks to my friends from Galgos laboratory and WhileTrue for the thoughts and jokes.

Finally, I would like to thank all the teachers and staff at PUC-Rio and the Department of Informatics for being part of this amazing institution that supports my intellectual and personal growth and the PUC-Rio and CNPq for the financial support.

In times we need the most is when we can clearly perceive that everything we do comes back to us. I counted with the help of many people on things that I thought would be impossible to do. I thank everyone who helped me in this incredible journey. 


\section{Abstract}

Silva, Thuener; Poggi, Marcus Vinicius(Advisor); Valladão, Davi Michel. Optimization Under Uncertainty for Asset Allocation. Rio de Janeiro, 2015. 99p. DSc Thesis - Departamento de Informática, Pontifícia Universidade Católica do Rio de Janeiro.

Asset allocation is one of the most important financial decisions made by investors. However, human decisions are not fully rational, and people make several systematic mistakes due to overconfidence, irrational loss aversion and misuse of information, among others. In this thesis, we developed two distinct methodologies to tackle this problem. The first approach has a more qualitative view, trying to map the investor's vision of the market. It tries to mitigate irrationality in decision-making by making it easier for an investor to demonstrate his/her preferences for specific assets. This first research uses the Black-Litterman model to construct portfolios. Black and Litterman developed a method for portfolio optimization as an improvement over the Markowitz model. They suggested the construction of views to represent an investor's opinion about future stocks' returns. However, constructing these views has proven difficult, as it requires the investor to quantify several subjective parameters. This work investigates a new way of creating these views by using Verbal Decision Analysis. The second research focuses on quantitative methods to solve the multistage asset allocation problem. More specifically, it modifies the Stochastic Dynamic Dual Programming (SDDP) method to consider real asset allocation models. Although SDDP is a consolidated solution technique for large-scale problems, it is not suitable for asset allocation problems due to the temporal dependence of returns. Indeed, SDDP assumes a stagewise independence of the random process assuring a unique cost-to-go function for each time stage. For the asset allocation problem, time dependency is typically nonlinear and on the left-hand side, which makes traditional SDDP inapplicable. This thesis proposes an SDDP variation to solve real asset allocation problems for multiple periods, by modeling time dependence as a Hidden Markov Model with concealed discrete states. Both approaches were tested in real data and empirically analyzed. The contributions of this thesis are the methodology to simplify portfolio construction and the methods to solve real multistage stochastic asset allocation problems.

\section{Keywords}

Portfolio Selection; Multistage Asset Allocation; Investments Analysis; Decision support systems; Black Litterman; Stochastic Dual Dynamic Programming. 


\section{Resumo}

Silva, Thuener; Poggi, Marcus Vinicius; Valladão, Davi Michel. Otimização Sob Incerteza para Alocação de Ativos. Rio de Janeiro, 2015. 99p. Tese de Doutorado - Departamento de Informática, Pontifícia Universidade Católica do Rio de Janeiro.

A alocação de ativos é uma das mais importantes decisões financeiras para investidores. No entanto, as decisões humanas não são totalmente racionais. Sabemos que as pessoas cometem muitos erros sistemáticos como, excesso de confiança, aversão à perda irracional e mau uso da informação entre outros. Nesta tese desenvolvemos duas metodologias distintas para enfrentar esse problema. A primeira abordagem é qualitativa, utiliza o modelo de Black-Litterman e tenta mapear a visão que o investidor tem do mercado. Esse método tenta mitigar a irracionalidade na tomada de decisão tornando mais fácil para um investidor demonstrar suas preferências em relação aos ativos. Black e Litterman desenvolveram um método para otimização de carteiras com a proposta de melhorar o modelo Markowitz, utilizando a construção de visões para representar a opinião do investidor sobre o futuro. No entanto, a forma de construir essas visões é bastante confusa e exige que o investidor estime vários parâmetros que são subjetivos. Assim, propomos uma nova forma de criar essas visões, utilizando Análise Verbal de Decisão. A segunda pesquisa envolve métodos quantitativos para resolver o problema de alocação de ativos com múltiplos estágios com premissas mais realistas. Embora a Programação Dinâmica Dual Estocástica (PDDE) seja uma técnica promissora para a solução de problemas de grande porte, não é adequada para o problema de alocação de ativos devido à dependência temporal associada aos retornos dos ativos. PDDE assume que o processo estocástico tem independência por estágio assegurando uma função única de custo futuro para cada estágio. No problema de alocação de ativos, a dependência do tempo é tipicamente não-linear e no lado esquerdo, o que torna PDDE tradicional não aplicável. Propomos uma variação do PDDE usando modelo oculto de Markov com estados discretos para resolver problemas reais de alocação de ativos com múltiplos períodos e dependência no tempo. Ambas as abordagens foram testadas em dados reais e empiricamente analisadas. As principais contribuições são as metodologia desenvolvidas para simplificar a construção de portfólios e para resolver o problema de alocação de ativos com múltiplos estágios.

\section{Palavras-chave}

Seleção de Carteiras; Alocação de Ativos em multi-estágio; Análise de Investimentos; Método de Apoio à Tomada de Decisão; Black Litterman; Programação Dinâmica Dual Estocástica. 


\section{Contents}

1 Introduction $\quad 13$

1.1 Objective 13

1.2 Contributions 14

$\begin{array}{lll}1.3 & \text { Outline } & 15\end{array}$

1.4 Assumptions and Notation 15

2 Asset Allocation $\quad 17$

$\begin{array}{lll}2.1 & \text { Utility function } & 17\end{array}$

2.2 Mean-Variance Model 18

2.3 Modern Asset Allocation Methods 19

3 More Human-like Portfolio Optimization Approach 21

3.1 Introduction 21

3.2 Verbal Decision Analysis 23

Formal Statement of the Problem $\quad 25$

The ZAPROS-III Method $\quad 25$

3.3 Black-Litterman 26

Market Equilibrium $\quad 27$

Specifying Views $\quad 29$

The Estimation Model $\quad 30$

$\begin{array}{ll}\text { Idzorek } & 31\end{array}$

3.4 Experiments with Brazilian stocks 31

Construction of the Views 31

Results 32

4 Dynamic Asset Allocation Under Uncertainty 39

$\begin{array}{lll}4.1 & \text { Introduction } & 39\end{array}$

4.2 Stochastic Dynamic Dual Programming 41

Risk Neutral SDDP 42

Time Consistent Risk Averse Model 43

Conditional value at risk 44

Stopping Criteria $\quad 46$

$\begin{array}{ll}\text { Sampling Scenarios } & 47\end{array}$

4.3 Stochastic Dynamic Programming for Asset Allocation 49

Myopic policy: No transaction costs and temporal independence 50

SDDP for asset allocation: Transaction costs and temporal independence $\quad 51$

Transactional costs $\quad 52$

H2SDDP for allocation: Transaction costs and temporal dependence 53

H2SDDP 54

Robust H2SDDP for asset allocation: Transaction costs, temporal dependence and ambiguity aversion 61 
H2SDDP for asset allocation: Transaction costs, temporal dependence and sell short

4.4 Computational Experiments $\quad 64$

Data analysis $\quad 64$

$\begin{array}{ll}\text { Sampling Methods } & 67\end{array}$

Sensitivity Analysis $\quad 68$

HMM 69

Impact of the Risk Aversion $\quad 69$

$\begin{array}{ll}\text { Convergence and trials } & 70\end{array}$

Transactional costs $\quad 71$

Models Evaluation $\quad 73$

Experiment with Monthly Data Set 2012 to 2014

Experiment with Monthly Data 2007 to $2014 \quad 76$

Experiment with Daily Data Set 80

5 Conclusions and Future Works $\quad 82$

$\begin{array}{lr}\text { Bibliography } & 86\end{array}$

$\begin{array}{ll}\text { A Appendix } & 95\end{array}$

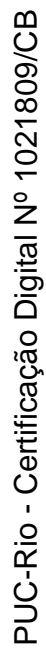

A.1 Questionnaires $\quad 95$

A.2 Myopic prove $\quad 96$ 


\section{List of Figures}

3.1 Procedure to apply ZAPROS-III methodology 26

3.2 Flowchart of Black-Litterman method [19] 27

3.3 The Black-Litterman portfolio with our views 35

3.4 Equilibrium portfolio 35

3.5 Result of the increase in the qualification of Sid. Nacional 36

3.6 Result of the increase in the qualification of $\mathrm{Oi} 36$

3.7 Result of right scenario, where the investor guess is right 38

3.8 Result of wrong scenario, where the investor guess is wrong 38

4.1 -CVaR and -VaR for gain distribution 45

4.2 Example of Latin Hypercube Sampling for uniform distribution with two dimensions $\quad 48$

4.3 Sampling with Monte Carlo 49

4.4 Sampling with Latin Hypercube Sampling 49

4.5 LHS interval for a normal distribution $N \sim(0,1) \quad 49$

4.6 An example of HMM for stock market 56

4.7 An example of a Gaussian mixture model. 56

4.8 Decision tree of the generic problem with return dependence 60

4.9 Decision tree of the problem with return dependence modeled with HMM 60

4.10 Decision tree of the problem of our proposal 61

4.11 Metrics for historical monthly return series of the five industrial portfolios

4.12 Metrics for historical daily return series of the ten industrial portfolios

4.13 Cumulative performance for monthly data set of the five industrial portfolios

4.14 Cumulative performance for daily data set of the ten industrial portfolios

4.15 Comparison between Latin Hypercube Sampling (LHS) and Monte Carlo (MC)

4.16 States likelihood for train data

4.17 Impact of the risk aversion coefficient $(\lambda)$ on the upper bound for monthly returns and daily returns, respectively on the left and right

4.18 Convergence of the Upper Bound for monthly data set 71

4.19 Impact of the transactional costs on the upper bound $\quad 72$

4.20 Portfolio allocation without transactional costs 73

4.21 Portfolio allocation with transactional costs 73

4.22 Comparing the different methods for asset allocation data form 2012 to 2014

4.23 Cumulative returns series from January 2012 to December 2014

4.24 Returns series from January 2007 to December 2014 
4.25 Comparing the different methods for asset allocation data form 2007 to 2014

4.26 Comparing the market states with the performance of the H2SDDP for data form 2007 to 2014

4.27 Trailing returns for asset allocation methods with monthly data form 2007 to 2014

4.28 Comparing the performance of H2SDDP with sell short sell short with other methods, monthly data form 2007 to 2014

4.29 Trailing returns of H2SDDP with sell short sell and other methods, monthly data form 2007 to 2014

4.30 Cumulative performance for asset allocation methods with daily data set form 2007 to 2014

4.31 Trailing returns for asset allocation methods with daily data set form 2007 to 2014 


\section{List of Tables}

$\begin{array}{lll}1.1 & \text { Set and stochastic process notation } & 15\end{array}$

1.2 State and decision variable notation 16

$\begin{array}{lll}2.1 & \text { Some properties of the utility function } & 18\end{array}$

3.1 FIQ and sector of the stocks 33

3.2 FIQ of the sectors 33

3.3 The expected return of the stocks 34

3.4 A summary of the views data 34

3.5 stocks returns for the period 37

3.6 Sectors returns for the period 37

3.7 Return for the different scenarios and the Market Portfolio 38

4.1 Monthly data series for 5 industrial portfolios 65

4.2 Daily data series for 10 industrial portfolios 65

4.3 Number of stages and the computational time 70

4.4 CVaR and return values of the simulated polices for different risk coefficients, results in percentage 74

A.1 Questionnaire about the stocks 95

A.2 Questionnaire about the sectors 96 


\section{Introduction}

Asset allocation is one of the most important financial decisions made by investors. The asset allocation problem consist in finding a portfolio (for example stocks, bonds, cash and gold) that better suits the investor's needs. Selecting good portfolios represents a competitive advantage, and to make good decisions in this regard it is necessary to leave emotions aside.

Emotions often affect investment decisions. In most cases, human decisions are not fully rational and people often make systematic mistakes due to overconfidence, irrational loss aversion and misuse of information. Such mistakes, made frequently by investors, can lead to big financial losses, which is one of the reasons why the behavioral finance field is dedicated to analyzing the psychology of financial decision making. Hence the need for tools that support the investors' financial decisions and prevent pitfalls.

Investment analysis techniques can be used as a tool or as automated optimization models to minimize, as much as possible, irrational intervention in decision making. In the last decades, there has been a remarkable increase in the use of financial models and optimization techniques for asset allocation. One of the main reasons for this is the attractive assumption that it is possible to forecast the conditional moments of the return distributions [1]. Another reason is the growth in processing power and the development of methods and optimization solutions that can handle a large volume of data. The field hardly existed in 1980, but has experienced a rapid surge ever since. Every day more tools are used to support the creation of investment strategies, and currently there is a large variety of approaches to the problem; Robust Optimization, Stochastic Programming and Machine Learning being only a few of the many fields in which we can encounter solutions to assist in financial decision making.

\subsection{Objective}

The main objective of this thesis is to help investors solve real asset allocation problems. In this regard, it presents two alternatives that aim at helping financial decision making.

The first approach has a qualitative perspective, trying to map the investor's vision of the market. It is an attempt to mitigate irrationality 
in decision making, making it easier for investors to demonstrate reasoned preferences concerning assets. For this purpose, the method combines the Black-Litterman portfolio optimization method with verbal decision analyses.

However, even when using decision-support methods, we are not safe from irrational decisions. That is why this work proposes quantitative methods using stochastic models to evaluate and solve the multistage asset allocation problem. More specifically, it suggests modifying the Stochastic Dynamic Dual Programming method to consider real asset allocation models.

\subsection{Contributions}

The major contributions of this work are derived from the two proposed methodologies. The first part of this thesis contributes by developing a simple new methodology that fits the investor's needs based on verbal decision analyses and Black-Litterman. This work has shown that it is possible to optimize portfolios even when the investor is not an expert on the subject. In addition, this approach makes it easier for the investors to manifest their own opinion, in an organized fashion, and allows them to change their portfolios more frequently. Finally, a case study based on Brazilian stocks demonstrates that this methodology creates more intuitive and diversified portfolios.

The second part of this thesis proposes a new approach to solve multistage stochastic asset allocation problems with time dependency. The method maps the temporal dependence as hidden Markovian states, transforming them into a convex problem that can be solved by adapting the SDDP. In addition, it presents a more general model that consider the ambiguity on the states' probabilities of the optimization problem. As our experiments demonstrate, the proposed model performs very well and shows promising results.

Main contributions:

- Proposes a new way to generate the Black-Litterman views using VDA, enabling the investor to construct personalized portfolio based on the his/her opinion.

- Model the multistage stochastic portfolio optimization problem with hidden Markovian temporal dependence and transactional cost.

- Create a more general model for the multistage stochastic portfolio optimization problem with ambiguity aversion, with the intent to mitigate returns estimation errors. 


\subsection{Outline}

This work is organized as follows: Chapter 2 introduces asset allocation and gives an overview of the methods that have been used to approach this problem. Chapter 3 is dedicated to the first proposed methodology, providing an overview of the Verbal Decision Analysis and Black-Litterman, and of how these two methods are combined. It also presents experiments conducted in the Brazilian stock market and some remarks about the proposed methodology. Chapter 4 describes the second, more quantitative, methodology proposed in this work. Its first part introduces the concept of SDDP and explains how this method may be adapted for asset allocation, while its final part proposes alternative models and shows various computational experiments. Chapter 5 brings this thesis' final conclusion and debate regarding future works, presenting the main contributions of the two proposed methodologies and suggestions for future works.

\subsection{Assumptions and Notation}

In this section, we present some assumptions and notation used through this thesis. It will be used bold-faced upper $(\boldsymbol{\Sigma}, \boldsymbol{\Pi}, \mathbf{Q}, \mathbf{P}, \ldots)$ and lowercase $(\boldsymbol{\mu}$, $\mathbf{p}, \mathbf{r}, \ldots)$ letters to denote, respectively matrices and vectors [2]. To simplify the formulations it will be use a vector with all elements equal one with proper dimension $\mathbb{1}=[1, \ldots, 1]^{\top}$.

The multistage problem has a finite planning horizon $T$; the probability space is $(\Omega, \mathcal{F}, \mathbb{P})$ with filtration $\mathcal{F}$, where $\mathcal{F}=\{\emptyset, \Omega\}$ and $\mathcal{F}=\mathcal{F}_{T}$. A specific notation for the portfolio selection application was created and is shown in Table 1.1 and Table 1.2.

\begin{tabular}{|c|c|}
\hline \multicolumn{2}{|r|}{ Sets } \\
\hline $\begin{array}{l}\mathcal{A}=\{1, \ldots, A\}: \\
\mathcal{H}=\{0, \ldots, T-1\}:\end{array}$ & $\begin{array}{l}\text { Index set of the } A \geq 1 \text { assets. } \\
\text { Set of stages. }\end{array}$ \\
\hline \multicolumn{2}{|r|}{ Stochastic Process } \\
\hline$r_{t i}(s):$ & $\begin{array}{l}\text { Excess return of asset } i \in \mathcal{A} \text {, between stages } t \in \\
\{1, \ldots, T\} \text { and } t-1 \text {, under scenario } s \in \Omega \text {, where } \\
\mathbf{r}_{t}(s)=\left(r_{1, t}(s), \ldots, r_{A, t}(s)\right)^{\top} \text { and } \mathbf{r}_{\left[t^{\prime}, t\right]}(s)= \\
\left(\mathbf{r}_{t^{\prime}}(s), \ldots, \mathbf{r}_{t}(s)\right)^{\top} \text { for } t^{\prime} \leq t .\end{array}$ \\
\hline$\overline{\mathbf{r}}_{\left[t^{\prime}, t\right]}=\left(\overline{\mathbf{r}}_{t^{\prime}}, \ldots, \overline{\mathbf{r}}_{t}\right)^{\top}:$ & Realization sequence of the asset returns for $t^{\prime} \leq t$. \\
\hline$\overline{\mathbf{r}}_{[t]}=\overline{\mathbf{r}}_{[0, t]}:$ & Realization sequence of the asset returns for 0 to $t$. \\
\hline
\end{tabular}

Table 1.1: Set and stochastic process notation 


\section{State Variables}

$W_{t}(s)$ : Wealth at stage $t \in \mathcal{H} \cup\{T\}$ under scenario $s \in \Omega$

\section{Decision Variables}

$x_{t i}(s)$ : Amount invested in asset $i \in \mathcal{A}$, at stage $t \in \mathcal{H}$ under scenario $s \in \Omega$, where $\mathbf{x}_{t}(s)=\left(x_{1, t}(s), \ldots, x_{A, t}(s)\right)^{\top}$ and $\mathbf{x}_{\left[t^{\prime}, t\right]}(s)=$ $\left(\mathbf{x}_{t^{\prime}}(s), \ldots, \mathbf{x}_{t}(s)\right)^{\top}$ for $t^{\prime} \leq t$

Table 1.2: State and decision variable notation

Without loss of generality, the risk-free asset is represented as the first asset of the portfolio without excess return, i.e., for each scenario $r_{1, t}(s)=0$, for all $t \in \mathcal{H} \cup\{T\}$ and all $s \in \Omega$. 


\section{2 \\ Asset Allocation}

Before Markowitz's work [3] the concept of diversification was greatly simplified. People had an intuition that putting everything in one asset lead to largely different results, while distributing their money among many assets greatly reduced the chance of all having bad returns simultaneously.

The essence of portfolio optimization theory is based on diversification, as by combining different assets it is possible to obtain a lower risk than the offered by any of the assets individually. As the number of the assets increase, the variance of the portfolio decreases towards zero [1].

The Asset Allocation consists in finding the most appropriate group of assets while considering the individual properties of each asset. The optimal portfolio varies according to the profile of each investor, and there isn't a single portfolio that is recommended for every type of investor. This is due to the specific characteristics of each individual, institution or group. An investor averse to risk may prefer to invest in assets with low risk and low return, while another investor, who is more open to risk, might prefer assets with more risk when it is possible to achieve higher returns.

\subsection{Utility function}

To provide the most suitable portfolio for a given investor, it is necessary to understand its preference among the set of possible investments. A way to accomplish this is to map the utility function $U$ that captures investor's satisfaction or happiness at a given level of wealth. For a given level of wealth $W$, the usefulness $U(W)$ is the investor's satisfaction achieved with this wealth. Assuming that an investor with utility function $U$ attempts to make an investment portfolio and that $P\left(w_{s}\right)$ is the probability of this portfolio generating a wealth $w_{s}$ for a scenario $s$, we can calculate the expected utility for the portfolio as being:

$$
E[C]=\sum_{s} U\left(w_{s}\right) P\left(w_{s}\right)
$$

Utility functions have some important properties. First, the utility function should prefer more to less wealth, and therefore the utility of $X+1$ units 
is greater than that of $X$ units. This implies a positive first derivative of the utility function. The investor's attitude towards risk is also related to the utility function. Considering that when it comes to risk, an investor can be averse to it, neutral or like it.

The investor's classification can be defined by considering a fair bet, i.e. an investment where the value is equal to the expected cost. The investor that rejects a fair bet is risk averse, and thus its dissatisfaction with a loss is greater than its satisfaction with a gain for the same value. Functions with this behavior have a negative second derivative. The opposite occurs in the case of an investor who accepts a fair bet, a case in which the second derivative is positive. For an investor who is indifferent to risk, the utility of these two investments, with the same expected value, is the same, meaning the second derivative of this function is zero. Table 2.1 presents the characteristics of the second derivative according to investors' profiles.

\begin{tabular}{|c|c|c|}
\hline Profile & Behavior & Implication \\
\hline \hline Risk aversion & Rejects fair bet & $U^{\prime \prime}(W)<0$ \\
\hline Risk neutral & Indifferent to fair bet & $U^{\prime \prime}(W)=0$ \\
\hline Taste for Risk & accepted the fair bet & $U^{\prime \prime}(W)>0$ \\
\hline
\end{tabular}

Table 2.1: Some properties of the utility function

\subsection{Mean-Variance Model}

In his pioneering work "Portfolio Selection" [3], Markowitz developed a model that allows the selection of portfolios considering the relation between return and risk. This became known as the mean-variance model, which uses the expected return as a measure of performance of the portfolio and the variance as a risk measure.

A portfolio with $n$ assets can be represented by the amount invested on each asset $\mathbf{x}=\left(x_{1}, \ldots, x_{A}\right)$. Assuming that future returns are random variables and using each individual expected return, the portfolio return can be estimated by the equation

$$
E\left[R_{p}\right]=\sum_{i=1}^{A} x_{i} E\left[R_{i}\right]
$$

In order to evaluate the variance of portfolio $\sigma_{c}^{2}$, it is used the covariance of the return series between all pairs of assets that make up the portfolio $\sigma_{i j}, \forall i, j \in\{1, \ldots, A\}$. 


$$
\sigma_{p}^{2}=\sum_{i=1}^{A} \sum_{j=1}^{A}\left(x_{i} x_{j} \sigma_{i j}\right)
$$

Using the mean-variance approach, one can create an efficient frontier. That is the set of portfolios in which a given risk level has the highest possible return, or a certain level of return has the lowest possible risk. These are efficient portfolios. All other portfolios can be considered inefficient, because they have either lower return or higher risk.

Formally, the two minimization (2.1) and maximization (2.2) formulations are described below

$$
\begin{array}{ll}
\min _{\mathbf{x}} & \sum_{i=1}^{A} x_{i}^{2} \sigma_{i}^{2}+\sum_{i=1}^{A} \sum_{j=1, j \neq i}^{A} x_{i} x_{j} \sigma_{i j} \\
\text { s. t. } & \sum_{i=1}^{A} \mu_{i} x_{i} \geq r_{p} \\
& \sum_{i=1}^{A} x_{i}=1 \\
& x_{i} \geq 0, \forall i \\
\max _{\mathbf{x}} & \sum_{i=1}^{A} \mu_{i} x_{i} \\
\text { s. t. } & \sum_{i=1}^{A} x_{i}^{2} \sigma_{i}^{2}+\sum_{i=1}^{A} \sum_{j=1, j \neq i}^{A} x_{i} x_{j} \sigma_{i j} \leq v_{p} \\
& \sum_{i=1}^{A} x_{i}=1 \\
& x_{i} \geq 0, \forall i
\end{array}
$$

where the number of assets that may be part of the portfolio is $N$, and $x_{i}$ is the percentage of the portfolio that will be granted to asset $i$. The covariance between assets $i$ and $j$ is represented by $\sigma_{i j}$, and $\sigma_{i}^{2}$ is the variance of asset $i$. Finally, $\mu_{i}$ is the expected return of asset $\mathrm{i}$, and $r_{p}$ and $v_{p}$ are the minimum return and the maximum risk for the desired portfolio, respectively.

\subsection{Modern Asset Allocation Methods}

Even with several tools available to help investors create mean-variance optimized portfolios, they are still very skeptical about the mean-variance theory and its practical implications. Albeit revolutionary, Markowitz's work 
has shown major drawbacks in practical applications, yielding portfolios that can be counter-intuitive $[4,5]$, that tend to concentrate on a small subset of available assets and that do not seem well diversified $[6,7]$. The optimal portfolio is also extremely sensitive to small variations in the input data $[5,6,8]$.

This does not mean, however, that the mean-variance theory is flawed, but only that the idea needs to be remodeled or adapted in order to achieve better results. Hence, several new methodologies for portfolio optimization, and consequently for asset allocation, have been developed.

For instance: regarding the mean-variance's hypersensitivity to changes on the estimated inputs, which suggests that those parameters need to be estimated in an extremely precise way, several attempts to reduce the impact of estimation errors have been made. In fact, there are several ways to create portfolios that are less sensitive to these variations, such as shrinkage estimators, Bayesian and resampling methods and robust optimization $[4,5$, 9,10]. Frost and Savarino [11] have demonstrated that these optimization constraints stabilize the portfolio and generally improve performance. As mentioned by Jagannathan and Ma [12], these constrains can be interpreted as a posteriori regularization.

In this thesis, we will focus on two different approaches, presented in the following chapters. Considering the instability problems and parameterestimation method of the Markowitz model, we will propose a methodology to construct a portfolio based on the investors' opinion. Another approach is to focus on solving the asset allocation problem in a quantitative way. This will be modeled as a multistage stochastic problem with temporal dependence. 


\section{3}

\section{More Human-like Portfolio Optimization Approach}

\subsection{Introduction}

The practical disadvantages of the Markowitz model motivated Fisher Black and Robert Litterman to develop a new approach. Thus the BlackLitterman approach [13], which combines the expected equilibrium between returns estimated through the Capital Asset Pricing Model (CAPM) and views to optimize the portfolio. The views represent the investor's opinion about the stocks' future returns. This model yields more stable and diversified portfolios than the mean-variance standard model [14].

Black and Litterman's original paper [4] only explained the core aspects of their idea, leaving it to others to better explain the implication of their model. Satchell and Scowcroft [15], Walters [14], He and Litterman [16] explain the Black-Litterman solution in further detail. Walters [14] also constructed a framework ${ }^{1}$ to use the model and other portfolio optimization techniques. Mankert [17] sheds more light on the practical implications of the BlackLitterman approach. Other studies focus on extensions of the original model, like Herold [18], Idzorek [19], Fernandes et al. [20], and Meucci [21].

Also, Bertisimas et al. [2] proposed a more general extension of the original Black-Litterman model that can incorporate investor opinion about volatility and construct estimators for more general notions of risk. Reinterpreting the problem through inverse optimization Bertisimas et al. [2] extends the traditional model creating a approach that can combine a greater variety of views.

The expression of the investor's preferences can be seen as a decision making process. Traditionally, decision making scenarios involve the analysis of objects from several points of view and can be assisted by multi-criteria methodologies. These help generating knowledge about the decision context and, as a consequence, increase the confidence of those making decisions [22]. There are multi-criteria methods based either on quantitative or qualitative

\footnotetext{
${ }^{1}$ That is available in www.blacklitterman.org
} 
analysis of the problem, and choosing the best approach is a great challenge. Examples of problem-solving using quantitative methods can be found in Castro et al. [23], Toncovich et al. [24], and Pinheiro et al. [25]. Among those who apply qualitative methods, we have Mendes et al. [26], Tamanini et al. [27], Tamanini et al. [28], Tamanini et al. [29] and Castro et al. [30].

The Verbal Decision Analysis is based on multi-criteria problem-solving through qualitative analysis methods. One of the advantages of qualitative methods is that all the questioning in the process of eliciting preferences is made in the decision maker's native language. Moreover, verbal descriptions are used to measure preference levels. This procedure is psychologically valid, respecting the limitations of the human information processing system. This characteristic makes the incomparability cases [31] become almost unavoidable since the scale of preferences is purely verbal and consequently not an accurate way of estimating values. Therefore, the method may not be capable of achieving satisfactory results in some situations, presenting an incomplete solution to the problem.

Establishing views in the traditional quantitative way is not an easy task and an investor would need help from an expert in the process. That is why we chose a method to setting views using Verbal Decision Analysis (VDA). For this propose, we developed questionnaires that are intuitive and can be answered by anyone with basic knowledge of investment options without needing any further special training.

The purpose of this chapter is to develop a methodology that constructs a personalized portfolio based on the investor's opinions. Our problem is not a typical multi-criteria problem, being actually very different from normal VDA applications. This is one of the major difficulties that have to be overcome in order to create the Black-Litterman views. For this purpose, in the final part of Section 3.4 we compare the return of investing on the investor most preferred asset with our proposed approach.

Moreover, the objective pursued is a technique to support the creation of a personal portfolio based on an individual's opinion, preferences or view. Therefore, a comparison among performances of portfolios, in the present case, should only consider portfolios that are aligned with the considered individual preference. The technique proposed here follows the mean-variance balance of Markowitz generated portfolios.

In Section 3.2 we present a brief explanation of the Verbal Decision Analysis (VDA) framework used in this work. Section 3.3 brings a review of the Black-Litterman methodology. Finally, in Section 3.4 we report about the experiments made with Brazilian stocks. 


\subsection{Verbal Decision Analysis}

A decision may be defined as the result of a process of choice when someone is confronted with a problem or with an opportunity for creation, optimization or improvement of a given situation. On the other hand, decision making is a special activity of human behavior, aimed at the achievement of a given goal. It takes place in every activity of the human world, from simple daily problems to complex situations inside an organization. The conclusion of a decision making process can be an ordination of alternatives or the selection of a single alternative from a list of possible solutions for the problem.

Establishing its preferences and interests is usually enough to allow an individual to make decisions that solve simple problems. However, individuals often find it hard to separate emotions from reason. As a result, emotions often influence the decision making process $[32,33]$. The decision also involves several factors, some of which may not be measurable. Thus, when a decision maker needs to solve complex problems, covering many alternatives and a large volume of information that may not be measurable nor easily comparable, some methodologies exist to support the decision making process.

In order to solve a given problem, alternative solutions are taken into consideration. Such alternatives are defined and characterized according to a set of criteria, structured around its verbal and qualitative nature. There are a huge number of practical problems which is necessary to generate an ordinal scale of alternatives [34]. The construction of such an ordinal scale is helpful in many situations, for example, to reject less preferable alternatives from a given set.

The Verbal Decision Analysis (VDA) framework is a set of methods defined to support the decision making process through the verbal representation of problems. Some methods that constitute the Verbal Decision Analysis framework are: ZAPROS-III, ZAPROS-LM, PACOM, and ORCLASS Larichev and Moshkovich [34]. According to Gomes et al. [35], in the majority of multicriteria problems there is a set of alternatives that can be evaluated against the same set of characteristics (called criteria or attributes). The VDA framework is structured on the supposition that most decision making processes can be qualitatively described [36]. Although the decision maker's ability to choose is very dependent on the occasion and the stakeholders' interest, the methods to support decision making are universal.

Moreover, in Ustinovich and Kochin [37] the analysis of a large amount of data-processing performed by human beings has shown that the psychologically correct operations are: 
- Comparison of two assessments in verbal scale by two criteria;

- Assignment of multi-criteria alternatives to decision classes;

- Comparative verbal assessment of alternatives according to separate criteria.

This last operation is the only classification methodology within the VDA framework. The goal of the Verbal Decision Analysis framework is to establish a ranking of alternatives in order of preference.

The methods belonging to the Verbal Decision Analysis framework may be evaluated in light of their objectives:

- As a tool for ordinary classification, ORCLASS was one of the first methods designed to tackle classification problems. There are several other widely known methods for solving classification problems that can be applied and analyzed for future applications [38-40], but that does not belong to Ustinovich and Kochin's [37] VDA framework;

- The other objective is to organize the solutions alternatives for the problem in a rank, from the most preferable to the least preferable one. Three methods are proposed within the VDA framework: ZAPROS-LM, ZAPROS-III, and PACOM. Although they have the same final goal, they have different purposes:

- PACOM is exclusively created to be applied according to pair compensation and consists in comparing the advantages and disadvantages of multi-attribute alternatives.

- The ZAPROS method was created to be applied by pair comparison and consists in comparing a pair of alternatives with the advantage of reaching a decision by using simple and understandable dialogue. It is also divided in two alternative methods:

* ZAPROS-III differs from ZAPROS-LM in its level of treatment of inconsistence. ZAPROS-III can be considered an evolution of ZAPROS-LM in this concept. 


\section{(a) Formal Statement of the Problem}

The methodology follows the same problem formulation proposed by [34], where:

1. $\mathcal{K}=\left\{c_{1}, c_{2}, \ldots, c_{N}\right\}$, representing a set of $N$ criteria;

2. $n_{q}$ represents the number of possible values on the scale of q-th criterion, $(q \in \mathcal{K})$; For the ill-structured problems, as in this case, usually $n_{q} \leq 4$;

3. $\mathcal{X}_{q}=\left\{x_{1}, x_{2}, \ldots, x_{n_{q}}\right\}$ represents a set of values to the $q$-th criterion, which is this criterion scale; $\left|\mathcal{X}_{q}\right|=n_{q}$; The values of the scale are ranked from best to worst, and this order does not depend on the values of other scales;

4. $\mathcal{Y}=\mathcal{X}_{1} \times \mathcal{X}_{2} \times \cdots \times \mathcal{X}_{N}$ represents a set of vectors $\mathbf{y}_{i}$, in such a way that: $\mathbf{y}_{i}=\left\{y_{i 1}, y_{i 2}, \ldots, y_{i N}\right\}^{\top}$ and $\mathbf{y}_{i} \in \mathcal{Y}, y_{i q} \in X_{q}$, where $|\mathcal{Y}|=\prod_{q=1}^{N} n_{q}$;

5. $\mathcal{Z}=\left\{\mathbf{z}_{i}\right\}_{1}^{j}$ and $\mathbf{z}_{i} \in \mathcal{Y}$, where the set of $j$ vectors represents the description of the real alternatives.

The order of the multi-criteria alternatives on set $\mathrm{A}$ is defined based on the decision maker's preferences.

\section{(b) The ZAPROS-III Method}

According to Ustinovich and Kochin [37], one of the most important features of ZAPROS methods is the use of psychologically grounded procedures for identifying the preferences. This method evaluates personal abilities and limitations of human information processing system. The disadvantages of the method also include the limited amount of attributes and difficulties in using quantitative criteria.

Furthermore, ZAPROS-III [33] considers values known as Quality Variations (QV) or Quality Changing (QC) [36] and Formal Index of Quality (FIQ). The QV represents the distance between the evaluations of two criteria. The FIQ mainly aims at minimizing the number of comparable pairs of alternatives. The FIQ is used in the ranking of the alternatives.

Figure 3.1 [31] presents a flowchart with steps for the application of the VDA method ZAPROS-III. As described in the Figure 3.1, the method's application can be divided into four stages: Problem Formulation, Elicitation of Preferences/Comparison of Alternatives, Validation of the Decision maker's preferences, and Comparison of Alternatives. 


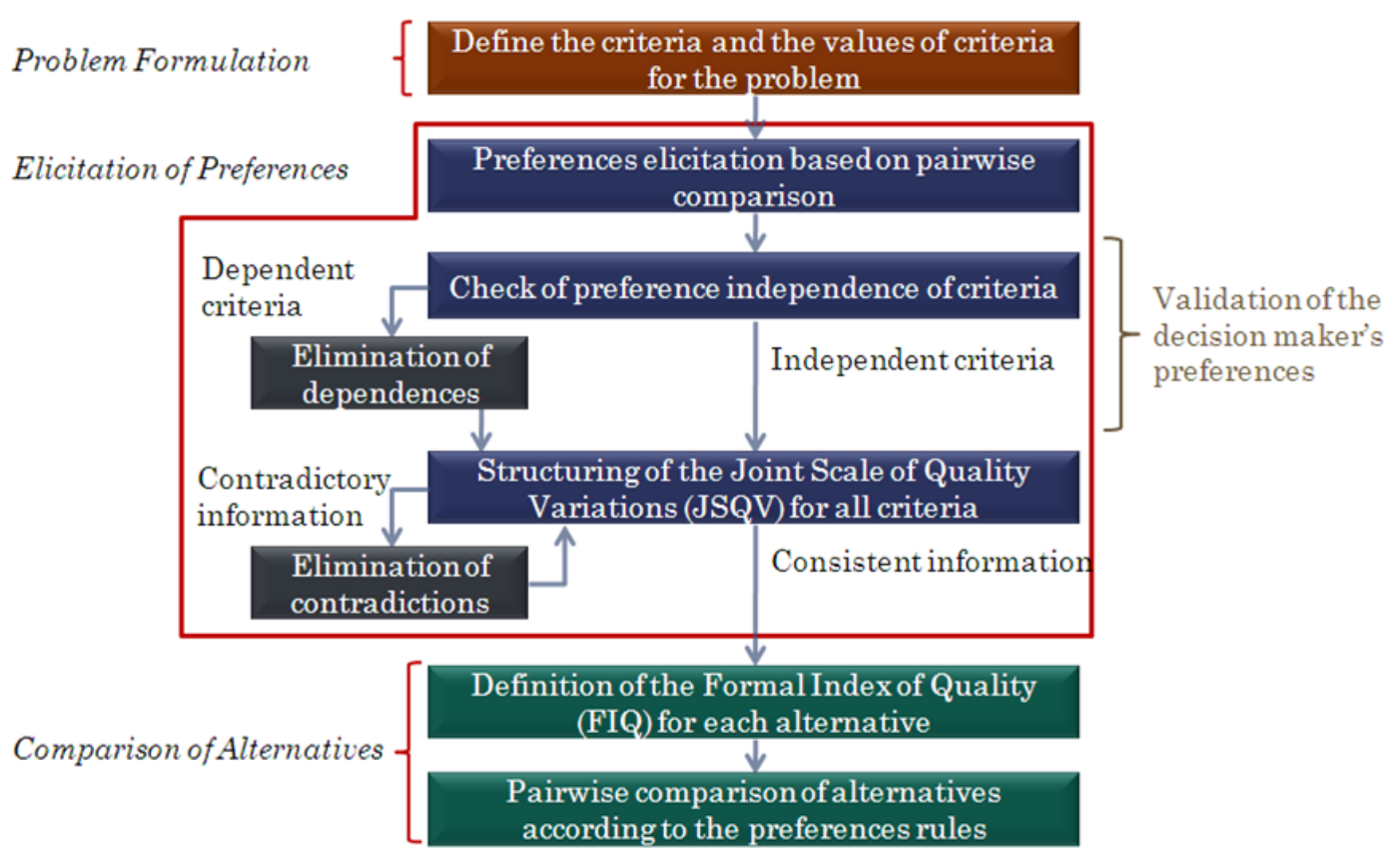

Figure 3.1: Procedure to apply ZAPROS-III methodology

A disadvantage of the method is that the number and values of criteria that can be handled are limited, in order to keep complexity under control.

Tamanini [31] defends that although ZAPROS-III-i follows a procedure similar to its predecessor's to extract preferences, it also implements modifications that make it more efficient and more accurate regarding inconsistencies. The number of incomparable alternatives is essentially smaller than in previous ZAPROS [36].

\subsection{Black-Litterman}

The traditional portfolio approach proposed by Markowitz has some issues and does not consider the investor's vision of the market. Hence, the Black-Litterman [13] was conceived to be a more practical and more flexible portfolio management method [41]. Its methodology begins by determining the equilibrium portfolio and the views of the investor, after these are combined to construct a new distribution of the stocks' returns. Using this new distribution, a portfolio optimization problem is formulated and a new optimal portfolio is obtained. A summary of the Black-Litterman model is present in Figure 3.2. 


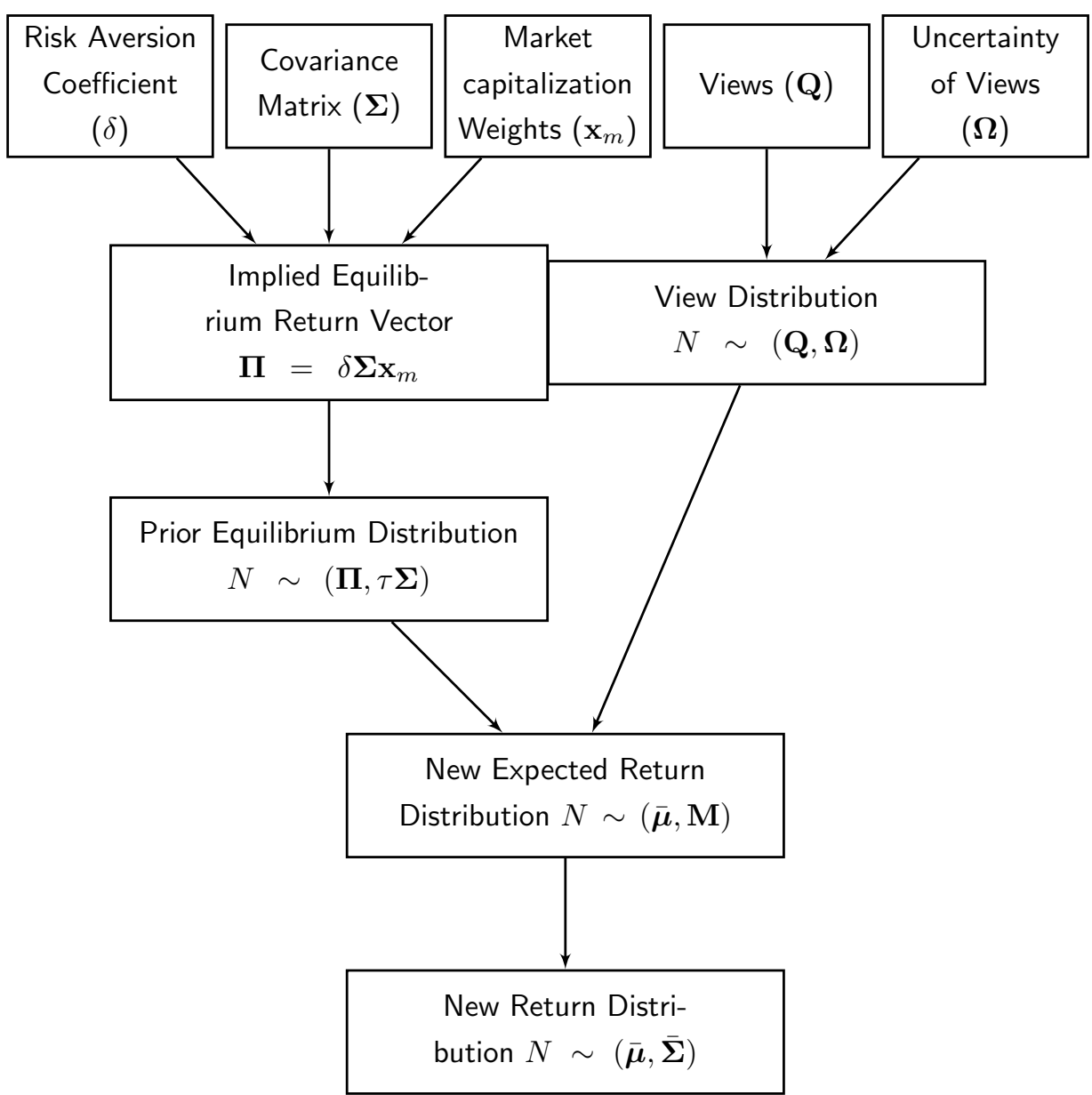

Figure 3.2: Flowchart of Black-Litterman method [19]

The model proposed by Black and Litterman can be seen, in a rather simplistic way, as an adjustment in the prior distribution of the assets' returns to adapt it to the investor's vision. Essentially, however, it combines the investor's views with the CAPM notion of market equilibrium $[4,13]$.

\section{(a) Market Equilibrium}

The Black-Litterman assumption is that the a priori distributions of returns are consistent with market equilibrium. Considering that all investors' utility functions are the same, the CAPM theory shows that everyone should hold the same portfolio, the market portfolio $\mathbf{x}_{m}$. The market portfolio is the portfolio where the amount of assets is proportional to its market value.

First we have to assume that the returns of the stocks $\mathbf{r}$ are normally distributed with mean $\mathbb{E}(\mathbf{r})$ and covariance matrix $\boldsymbol{\Sigma}$ i.e. $r \sim N(\mathbb{E}(\mathbf{r}), \boldsymbol{\Sigma})$. When the market is efficient, the expected return for any asset has the following property

$$
\mathbb{E}\left(r_{i}\right)-r_{f}=\beta_{i}\left(\mathbb{E}\left(r_{m}\right)-r_{f}\right)
$$


The $\mathbb{E}\left(r_{i}\right)$ and $\mathbb{E}\left(r_{m}\right)$ are the asset $i$ and market portfolio's expected returns, while $r_{f}$ is the risk free asset return. Coefficient $\beta_{i}$ is the covariance between asset $i$ and the market portfolio returns, divided by the market portfolio variance

$$
\beta_{i}=\frac{\sigma_{i m}}{\sigma_{m}^{2}}
$$

Also, the market portfolio return is

$$
r_{m}=\sum_{j=1}^{n} r_{j} x_{m j}
$$

The risk equilibrium premium $\boldsymbol{\Pi}$ is the expected excess of return yielded by the risky stocks, which should perform better than the risk free stock. It is properly defined as the difference between the asset returns and risk free returns $\Pi_{i}=\mathbb{E}\left(r_{i}\right)-r_{f}$. Using the fact that

$$
\sigma_{i m}=\sum_{j=1}^{n} x_{m j} \sigma_{j i}
$$

and (3.1) we have

$$
\begin{aligned}
\Pi_{i} & =\beta_{i}\left(\mathbb{E}\left[r_{m}\right]-r_{f}\right) \\
& =\frac{\sigma_{i m}}{\sigma_{m}^{2}}\left(\mathbb{E}\left[r_{m}\right]-r_{f}\right) \\
& =\frac{\mathbb{E}\left[r_{m}\right]-r_{f}}{\sigma_{m}^{2}}\left(\sum_{j=1}^{n} x_{m j} \sigma_{j i}\right)
\end{aligned}
$$

With the risk aversion parameter $\delta$

$$
\delta=\frac{\mathbb{E}\left[r_{m}\right]-r_{f}}{\sigma_{m}^{2}}
$$

the final result can be expressed in matrix form as

$$
\Pi=\delta \Sigma \mathbf{x}_{m}
$$

A more detailed demonstration of these equations and more about the CAPM theory can be found in [42] and [43]. The result above can also be obtained by deriving the traditional quadratic utility function of the meanvariance model, assuming that all investors solve this problem.

Finally, we can define the prior distribution as the real $\boldsymbol{\mu}$ return distribution with mean $\boldsymbol{\Pi}$ and variance $\tau \boldsymbol{\Sigma}$

$$
\begin{array}{r}
\boldsymbol{\mu}=\boldsymbol{\Pi}+\boldsymbol{\epsilon}_{\pi} \\
\boldsymbol{\epsilon}_{\pi} \sim N(0, \tau \boldsymbol{\Sigma})
\end{array}
$$


The $\tau$ is a small number that reflects the investor's uncertainty about prior return estimations [44]. It is the most confusing parameter of the model and has several different calibration approaches. Further ahead we shall present Idzorek's technique to eliminate $\tau$.

\section{(b) Specifying Views}

The views are the investor's vision regarding future market behavior. These views can be relative or absolute and need to be "fully invested". Hence, the sum of weights is zero for the relative view, and one for the absolute. An example of absolute view is "Stock $i$ will return $q_{1} \%$ " and of a relative view is "International stock will outperform domestic stock by $q_{2} \%$ ". Furthermore, the confidence has to be defined by the investor, and this will change how much the view will affect the portfolio weights. The investor's view can be expressed as

$$
\mathbf{P} \boldsymbol{\mu}=\mathrm{Q}+\boldsymbol{\epsilon}_{q}
$$

Where $\mathbf{P}$ is the perspective of the investor and $\mathbf{Q}$ specifies the expected return of each view. The $\boldsymbol{\epsilon}_{q}$ is an non-observable random and normally distributed vector with mean zero and a diagonal covariance matrix $\Omega$ that expresses the uncertainty of the views $\left(\boldsymbol{\epsilon}_{q} \sim N(0, \boldsymbol{\Omega})\right)$.

Considering $v$ as the number of views and $n$ the number of stocks, $\mathbf{P}$ will be a matrix $v \times n$ with $\mathbf{p}_{i}(i \in\{1, \ldots, v\})$ representing a vector with $\mathrm{n}$ elements, $\mathbf{Q}$ a vector with $v$ elements and $\Omega$ a $v \times v$ diagonal matrix

$$
\begin{array}{r}
\mathbf{P}^{\mathrm{T}}=\left[\mathbf{p}_{1}, \mathbf{p}_{2}, \mathbf{p}_{3}, \ldots, \mathbf{p}_{v}\right] \\
\mathbf{Q}^{\mathrm{T}}=\left[q_{1}, q_{2}, q_{3}, \ldots, q_{v}\right] \\
\boldsymbol{\Omega}=\left[\begin{array}{cccc}
\omega_{1} & 0 & \ldots & 0 \\
0 & \omega_{2} & \ldots & 0 \\
\vdots & \vdots & \ddots & \vdots \\
0 & 0 & \ldots & \omega_{v}
\end{array}\right]
\end{array}
$$

To better understand how to describe these views in matrix form, two views were created: one relative and other absolute. In the first view, stock one will outperform stock two by $1 \%$ and in the second view, stock three will have return $2 \%$.

$$
\left[\begin{array}{ccc}
1 & -1 & 0 \\
0 & 0 & 1
\end{array}\right] \overline{\boldsymbol{\mu}}=\left[\begin{array}{l}
0.01 \\
0.02
\end{array}\right]+\boldsymbol{\epsilon}_{q}
$$




\section{(c) The Estimation Model}

With the expected excess return and the views of the investor, it is possible to proceed to the next step of the Black-Litterman approach, which combines these two items. There are two ways to estimate the final model. The original Black-Litterman paper [13] references the Theil's Mixed Estimation model [45], but there is also a Bayesian approach. The first method was chosen because it is easy to understand. By applying the identity matrix $I$, the problem can be seen in the matrix form

$$
\left[\begin{array}{l}
\mathbf{I} \\
\mathbf{P}
\end{array}\right] \overline{\boldsymbol{\mu}}=\left[\begin{array}{l}
\mathbf{\Pi} \\
\mathbf{Q}
\end{array}\right]+\left[\begin{array}{l}
\boldsymbol{\epsilon}_{\pi} \\
\boldsymbol{\epsilon}_{q}
\end{array}\right]
$$

Constructing the auxiliary matrices $\mathbf{D}=\left[\begin{array}{l}\mathbf{I} \\ \mathbf{P}\end{array}\right], \mathbf{C}=\left[\begin{array}{c}\boldsymbol{\Pi} \\ \mathbf{Q}\end{array}\right]$ and $\boldsymbol{\epsilon}=\left[\begin{array}{c}\boldsymbol{\epsilon}_{\pi} \\ \boldsymbol{\epsilon}_{q}\end{array}\right]$ we can reformulate the problem as

$$
\mathrm{D} \bar{\mu}=\mathrm{C}+\boldsymbol{\epsilon}
$$

$$
\boldsymbol{\epsilon} \sim N(0, \mathbf{W}), \mathbf{W}=\left[\begin{array}{cc}
\tau \Sigma & 0 \\
0 & \Omega
\end{array}\right]
$$

Solving this system of equations using least squares, we have

$$
\begin{aligned}
\overline{\boldsymbol{\mu}} & =\quad\left(\mathbf{D}^{\mathrm{T}} \mathbf{W}^{-1} \mathbf{D}\right)^{-1} \mathbf{D}^{\mathrm{T}} \mathbf{W}^{-1} \mathbf{C} \\
& =\left[(\tau \boldsymbol{\Sigma})^{-1}+\mathbf{P}^{\mathrm{T}} \boldsymbol{\Omega}^{-1} \mathbf{P}\right]^{-1}\left[(\tau \boldsymbol{\Sigma})^{-1}+\mathbf{P}^{\mathrm{T}} \boldsymbol{\Omega}^{-1} \mathbf{Q}\right]
\end{aligned}
$$

The variance can also be adjusted to reflect the change in the return data. Hence, the variance of the returns relative to the new data is

$$
\begin{aligned}
\mathbf{M} & =\left(\mathbf{D}^{\mathrm{T}} \mathbf{W}^{-1} \mathbf{D}\right)^{-1} \\
& =\left[(\tau \boldsymbol{\Sigma})^{-1}+\mathbf{P}^{\mathrm{T}} \boldsymbol{\Omega}^{-1} \mathbf{P}\right]^{-1}
\end{aligned}
$$

With this value $\mathbf{M}$, the actual new variance $(\overline{\boldsymbol{\Sigma}})$ can be evaluated as $[16]$

$$
\bar{\Sigma}=\Sigma+\mathbf{M}
$$

The final step in this process is to solve the mean-variance model by using the posterior distribution of the Black-Litterman. Having the new vector of expected returns and the covariance matrix, the new optimal portfolio can be estimated using the standard mean-variance method

$$
\max \mathbf{x}^{\mathrm{T}} \overline{\boldsymbol{\mu}}-\frac{\delta}{2} \mathbf{x}^{\mathrm{T}} \overline{\boldsymbol{\Sigma}} \mathbf{x}
$$

The solution obtained using the first-order conditional is 


$$
\mathrm{x}^{*}=\frac{1}{\delta} \overline{\boldsymbol{\Sigma}}^{-1} \overline{\boldsymbol{\mu}}
$$

\section{(d) Idzorek}

Idzorek describes an easy way to determine the level of trust by specifying the confidence level of each view as a percentage [19]. This method is deemed to be much more intuitive [14].

Another problem commonly found in the Black-Litterman model is the determination of $\tau$ [44]. Idzorek calibrates the confidence of a view so that $\mathbf{x} / \tau$ ratio is equal to the variance of the portfolio view $\left(\mathbf{p}^{\mathrm{T}} \mathbf{\Sigma} \mathbf{p}\right)$ [46], rendering the scalar value of $\tau$ irrelevant. Idzorek still presents his formulas with $\tau$, but it can be removed in order to simplify the equations [14].

\subsection{Experiments with Brazilian stocks}

Our process of composing a portfolio is divided in two stages: VDA and Black-Litterman. In the first step, the investor must answer a series of questions which will be used to create the views which, in turn, will be used in the BlackLitterman to build the new portfolio. We created a methodology to construct the view of the Black-Litterman model by using these questionnaires.

\section{(a) Construction of the Views}

Two different sets of questions were prepared. One of them is used to identify what are the investor's preferences regarding specific sectors of the financial market, while the other aims at mapping the investor's perspective regarding the companies he/she intends to invest in.

The questionnaire about the sectors contains 3 questions. The first one on how the domestic scenario is favorable to that sector, the second essentially the same as the first but regarding the external scenario, and the last one on the growth expectation for that sector. It was conceived simple, so it can be answered by most people.

The other questionnaire, about the stocks, has 7 questions regarding risk, reliability, expected growth, innovation, profitability, management, and company employees. It was also conceived to be as simple as possible, comprising only a few questions.

In order to construct the views based on the answers given in the questionnaires, we use the FIQ of the ZAPROS-III method. We consider the FIQ as a rating through which we can quantify not only the classification of stocks, but also how much one stock is better than another one. The FIQ has 
to be transformed into a standard for the views, and the values are normalized between 0 and 1 to create an absolute view that represents the investor's perspective.

For questionnaires such as the one about sectors, in which an alternative represents multiple stocks, we chose to equally divide the value attributed to the sector among the stocks. For example, if the value of the sector is considered to be 0.5 and we have two stocks, each one will have a value of 0.25.

Because confidence is a parameter that is somewhat complicated to determine - even as a percentage -, we decided to insert one more question in the questionnaires, in order to gauge how confident the participant is with his/her answers, thus obtaining the confidence of the view. To discretize the values, this question has four possible answers (very little confidence, little confidence, reasonably confident and very confident), which are associated with $25,50,75$, and 100 percent of confidence, respectively.

The last parameter of the view is the expected return. To have sufficient impact on the portfolio, we chose $0.5 \%$ as its value. This value was chosen based on the expected return of the assets and would be better calculated automatically, but it was not possible to conceive a general formula which was appropriate for any case.

\section{(b) Results}

To better understand how this methodology would behave in practice, a test program was conceived to work with the Aranaú [31] and Akutan [14] frameworks. After the questionnaires have been filled out, the program generates a graphical report showing the optimal portfolio and its details.

The Black-Litterman analytical resolution of the optimal portfolio has some limitations: even while using a Lagrangian decomposition, like in Silva et al. [47], the resulted formulation still cannot assure that the stocks's percentages are positive. Because of this limitation, the Jay Walters framework has to be extended to solve the problems using the CPLEX ${ }^{2}$ solver.

We chose the 10 major companies negotiated in the Brazilian market ${ }^{3}$ : Petrobras, ItaúUnibanco, Bradesco, Banco do Brasil, Vale, Itaúsa, Eletrobras, Sid. Nacional, Cemig and Oi. For each of these companies, we chose the stock with the highest negotiated volume to construct our portfolio and define the corresponding sector. These companies operate in the following sectors: electricity, financial, mining, oil, gas and biofuels, steel mill and metallurgy and telecommunications.

\footnotetext{
${ }^{2}$ Version 12.4 .0 .0

${ }^{3}$ In 2012 according to Forbes
} 
After answering the questions, we obtained the FIQ values for the stocks Table 3.1 and for the sectors Table 3.2. The lower the FIQ, the better the alternative. Therefore, these values were normalized using the difference between the maximum values of the companies.

\begin{tabular}{c|c|c}
\hline Stock & Sector & FIQ Stock \\
\hline Petrobras & Oil, gas and biofuels & 19 \\
ItaúUnibanco & Financial & 15 \\
Bradesco & Financial & 26 \\
Banco do Brasil & Financial & 19 \\
Vale & Mining & 11 \\
Itaúsa & Financial & 31 \\
Eletrobras & Electricity & 39 \\
Sid. Nacional & Steel mill and Metallurgy & 31 \\
Cemig & Electricity & 31 \\
Oi & Telecommunications & 46 \\
\hline
\end{tabular}

Table 3.1: FIQ and sector of the stocks

The same normalization is done with the sector FIQ, but with the values being distributed for all the stocks in the sector.

\begin{tabular}{c|c}
\hline Sector & FIQ Sector \\
\hline Oil, gas and biofuels & 12 \\
Financial & 1 \\
Mining & 7 \\
Electricity & 3 \\
Steel mill and Metallurgy & 8 \\
Telecommunications & 6 \\
\hline
\end{tabular}

Table 3.2: FIQ of the sectors

The expected return were estimated as the mean of the daily returns for February 2013, and these values are shown in Table 3.3. The returns vary greatly, but this was not specifically for this month, as the Brazilian market was experiencing some instability. 


\begin{tabular}{c|c}
\hline Stock & Exp. Ret. \\
\hline Petrobras & -0.6107 \\
ItaúUnibanco & 0.1759 \\
Bradesco & -0.145 \\
Banco do Brasil & 0.4877 \\
Vale & -0.3962 \\
Itaúsa & 0.1924 \\
Eletrobras & -0.1139 \\
Sid. Nacional & -0.6274 \\
Cemig & 0.3786 \\
Oi & -0.5714
\end{tabular}

Table 3.3: The expected return of the stocks

Considering a confidence level of $75 \%$ for both the stocks and sector questionnaires. Finally the views are composed by the confidence level, the return and the normalized FIQ values for both the assets and the sectors, which can be seen summarized in Table 3.4.

\begin{tabular}{c|c|c}
\hline Stock & View sector & View stocks \\
\hline Petrobras & 0.14 & 0.00 \\
ItaúUnibanco & 0.16 & 0.08 \\
Bradesco & 0.10 & 0.08 \\
Banco do Brasil & 0.14 & 0.08 \\
Vale & 0.18 & 0.14 \\
Itaúsa & 0.08 & 0.08 \\
Eletrobras & 0.04 & 0.13 \\
Sid. Nacional & 0.08 & 0.11 \\
Cemig & 0.08 & 0.13 \\
Oi & 0.00 & 0.17 \\
\hline \hline Confidence & $75 \%$ & $75 \%$ \\
Return & -0.0013 & -0.00083 \\
\hline Table $3.4 \mathrm{~A}$ &
\end{tabular}

Table 3.4: A summary of the views data

Inputting the calculated views into the Black-Litterman, we obtain the optimal portfolio of the Figure 3.3. To analyze how the portfolio changes, the equilibrium portfolio is presented in Figure 3.4. 


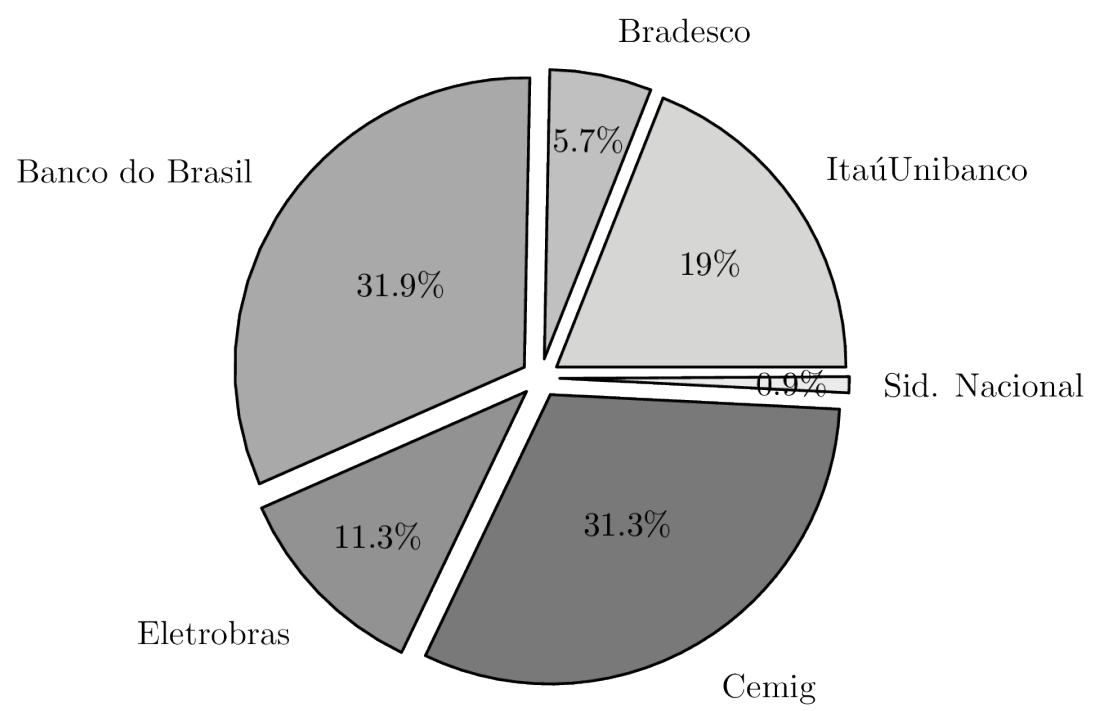

Figure 3.3: The Black-Litterman portfolio with our views

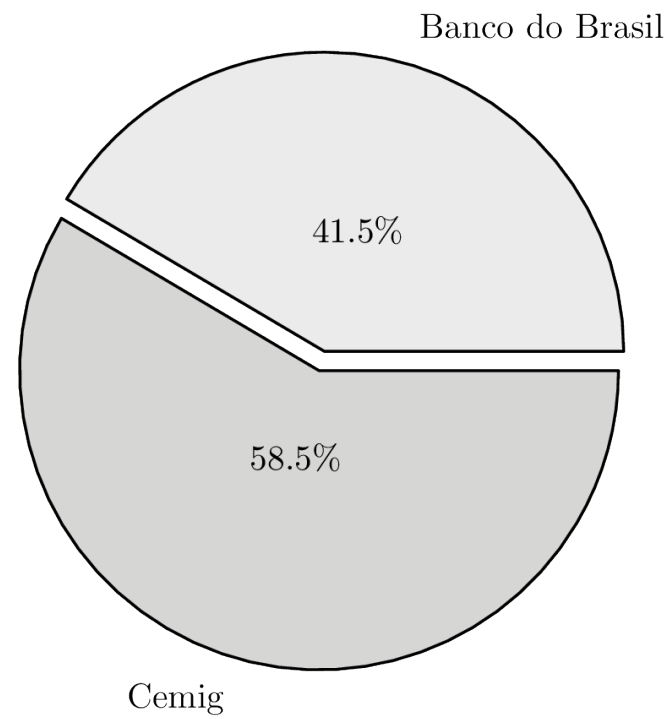

Figure 3.4: Equilibrium portfolio

To analyze the sensibility of our method, we conducted some experiments, as we shall see. However, we must emphasize that an improvement in the qualification of an asset does not necessarily mean an increase of its percentage in the optimal portfolio, as this variation also depends on the correlation and on the assets' return rates.

Answering the questionnaires with better expectations regarding the growth, the risk, the innovation, the profitability and the employees of Sid. Nacional, we obtained the portfolio in Figure 3.5. The participation of Sid. Nacional's stocks in the portfolio increased from $0.9 \%$ to $8.6 \%$. The increment was small because of the stock's equilibrium return and high correlation with Eletrobras. 


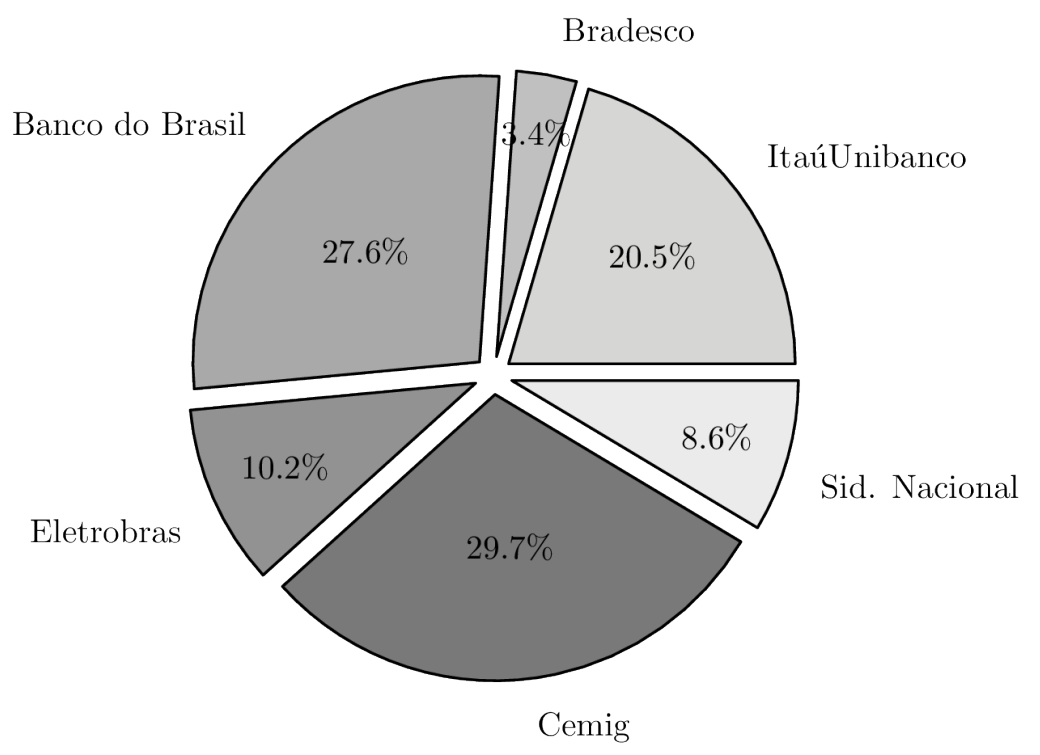

Figure 3.5: Result of the increase in the qualification of Sid. Nacional

The same thing happens if we increase the qualification of Oi, as seen in Figure 3.6. In this case, the correlation between Oi and Bradesco is negative, which explains why Bradesco's percentage also increases.

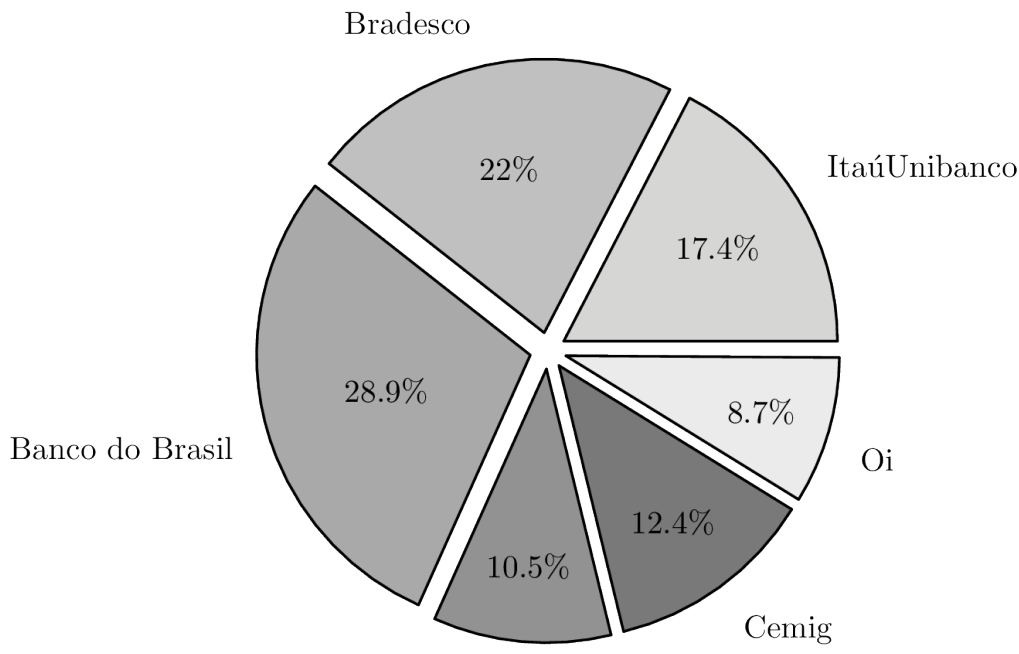

Eletrobras

Figure 3.6: Result of the increase in the qualification of Oi

We have similar behavior when we increase the qualifications of the sectors, but in this case the change is less significant due to the return of the sectors' view and because the increase is distributed among all the sector's stocks.

To analyze how the resultant portfolio would perform in different situations it was simulated two different views considering the future return of the stocks. It is assumed that the investor answers the questionnaires knowing the asset that will have the highest return and that is the only asset that he/she 
wants to invest. In the first scenario we have the best possible outcome, i.e. the investor guess was right and he/she invested on asset with highest return. In the second scenario the asset behavior contrary of what the investor was expecting, resulting on the worst portfolio return among all.

The performance was evaluated for a period of 6 months from February to September of 2013. In Table 3.5 it is presented the returns of the stocks for this period and Table 3.6 the returns for the sectors.

Table 3.5: stocks returns for the period

\begin{tabular}{c|c}
\hline Asset & Ret. (\%) \\
\hline Petrobras & 4 \\
ItaúUnibanco & -6 \\
Bradesco & -13 \\
Banco do Brasil & -7 \\
Vale & -7 \\
Itausa & -7 \\
Eletrobras & -18 \\
Sid. Nacional & -2 \\
Cemig & -3 \\
Oi & -44 \\
\hline
\end{tabular}

Table 3.6: Sectors returns for the period

\begin{tabular}{c|c}
\hline Sector & Ret. (\%) \\
\hline Oil, gas and biofuels & 4 \\
Financial & -8 \\
Mining & -7 \\
Electricity & -10 \\
Steel mill and Metallurgy & -2 \\
Telecommunications & -44 \\
\hline
\end{tabular}

Considering these values for the first scenario the highest asset return is Petrobras and the sector with the highest return is Oil, gas and biofuels. For the second scenario the worst asset return is $\mathrm{Oi}$ and the sector with the worst return is Telecommunications. Resultant portfolios obtain by answering the questionnaires considering those scenarios are presented in Figure 3.7 and 3.8 .

In Table 3.7 also compare these two portfolios with the Market Portfolio (optimized portfolio with the best Sharpe Ratio) and the portfolio generated before (Previous).

Analyzing the worst scenario it is evident the problem of allocating the portfolio entirely in an active disregarding the risk. If the investor had invested everything on Oi he/she would have lost $44 \%$ of its initial investment, using 


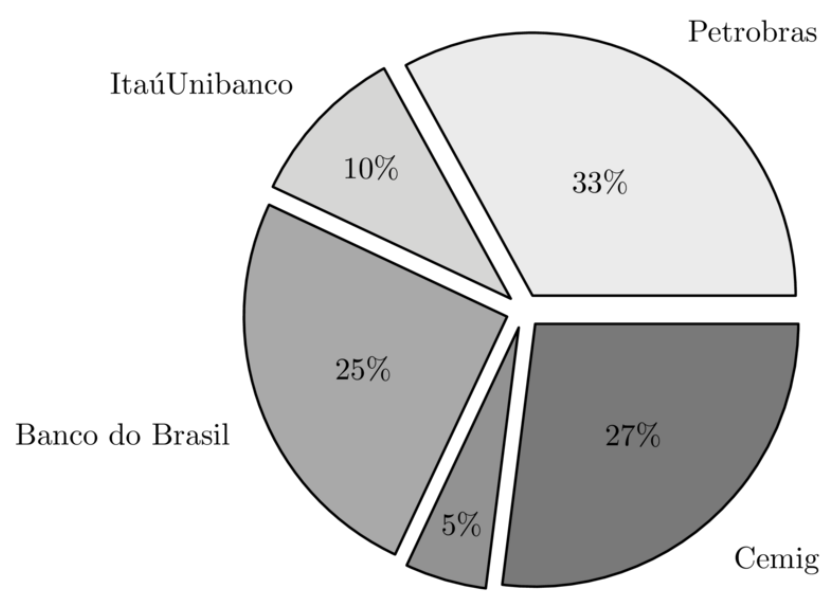

Eletrobras

Figure 3.7: Result of right scenario, where the investor guess is right

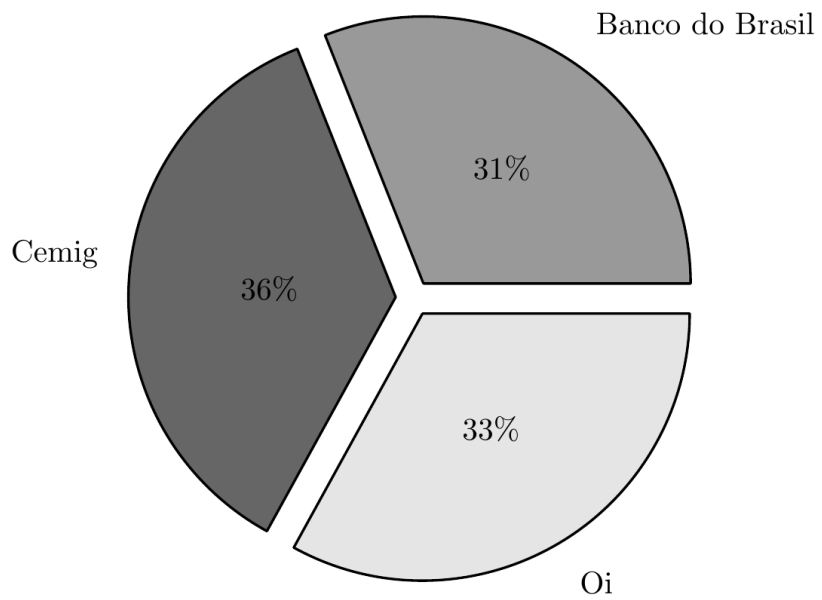

Figure 3.8: Result of wrong scenario, where the investor guess is wrong

Table 3.7: Return for the different scenarios and the Market Portfolio

\begin{tabular}{l|r}
\hline Portfolio & Ret. (\%) \\
\hline Previous & 93.04 \\
Market Portfolio & 95.19 \\
Right Scenario & 97.46 \\
Wrong Scenario & 82.35 \\
\hline
\end{tabular}

the methodology proposed in this article that loss would decrease to $18 \%$. However, a gain that would have invested in investor Petrobras decreases from $4 \%$ to $-3 \%$. These results indicate a decreased risk of the portfolio. 


\section{4 Dynamic Asset Allocation Under Uncer- tainty}

In real life applications, we deal with decision-making processes marked by uncertainty, i.e., with choices having to be made considering future realizations of risk factors. Stochastic Programming aims at finding optimal solutions for such cases involving uncertain data. These decisions also have to be continuously and periodically re-evaluated, which is why real life applications are often modeled as multistage stochastic problems.

However, there are many challenges in modeling and solving this type of application in order to avoid the curse of dimensionality, which is the major obstacle for multistage stochastic decisions. In fact, in several cases analysts have to deal with a very large number of possible outcomes at each stage and the scenario trees grow exponentially with the number of stages. Thus, as the problems grow, the number of scenarios can easily become enormous.

\subsection{Introduction}

There are two common types of methods that can be implemented to solve multistage stochastic problems. Methods for scenario decomposition, like progressive hedging and L-shaped (nested Benders); and sampling-based decomposition methods, such as Stochastic Dynamic Dual Programming (SDDP) [48], Abridged Nested Decomposition (AND) [49] and Convergent CuttingPlane and Partial-Sampling Algorithm (CUPPS) [50].

It is important to highlight that in most cases only sampling-based methods can efficiently solve problems with a large number of stages, as even for modern processing power other methods still require a huge computational effort. Hence, when dealing with large instances, sampling-based algorithmis are the most appropriate approach.

Pereira and Pinto [48] introduces the SDDP method, through a scenario sampling process it adds new constraints on the problem using the dual optimal solution. To overcome the curse of dimensionality, it assumes temporal independence to separate cost-to-go functions by stage. Sometimes, however, it is not reasonable to assume stagewise independence for some kind of problems. 
Therefore, if the problem presents some level of temporal dependence, the challenge is to modify the SDDP methodology keeping the cost-to-go function independent by stage.

Some problems with temporal dependence in the right-hand side, such as the hydrothermal scheduling, are treatable augmenting the state space [51]. In the hydrothermal model, for example, the rivers' inflows are time-dependent and, in order to create the independence between stages, the proposed solution is to use the inflow as state variable [52] [53]. To represent this linear correlation, it is also necessary to change the right-hand side accordingly. However, this approach is specific for problems with right-hand side linear dependence.

Mo et al. [54] and Philpott and Matos [55] have shown that when the relationships between stages are nonlinear, the problem can also be modified to maintain stagewise independence. Using the Markov chains to model the nonlinear temporal dependence, it is also possible to preserve stagewise independence between cost-to-go functions. As in the other methods mentioned above, the downside of these approaches is to increase the state space, thus raising the computational effort needed.

Furthermore, it is well-known that in financial time series returns also have a nonlinear dependence $[56,57]$. Portfolio optimization problems can be modeled as multistage stochastic problems in which for each stage a decision has to be made regarding where to invest. Therefore, multistage problems concerning asset allocation have temporal dependence on the left-hand side and are usually solved using scenario trees like in Valladão et al. [58]. However, not all the uncertainty regarding a problem can be represented using scenario trees, because it would become computationally untreatable.

When Dantzig and Infanger [59] researched this problem in 1993, they used classical Benders decomposition to solve the multistage portfolio problem. Nonetheless, their method relied on multidimensional integration, and the issue of dependence between stages was stated but not resolved.

Assuming temporal independence and no transactional cost, the optimal multistage asset allocation solution is obtained by a myopic policy [60], allowing such problems to be easily solved by solving a 2-stage problem. However, when confronted with the reality of transaction costs and temporal dependence, the proof of optimal myopic policy is not valid.

Our proposal in this chapter is to solve practical multistage asset allocation problems with realistic assumptions. Modeling the temporal dependence as a Hidden Markov Model (HMM), using Gaussian mixture, where the state likelihood depends on historical returns. 
In SDDP, we will create a worth function for each Markov state and stage, with the states probabilities at each stage depending on past returns. The downside of this approach is that it increases the size of the state space. Normally, for financial series the states of the Markovian process are concealed. Consequently, the HMM - a well-established technique - has been used to predict returns and determine the states probabilities. Using the worth functions for each Markovian states this proposed methodology will solve an approximation of real problem.

Similar approach was used in Mo et al. [54] Philpott and Matos [55], although their approach are different, the dependence is on the right-hand side, while in the asset allocation problem it is on the left-hand side, and they have observable states.

In these dynamic decision problems, like multistage asset allocation, there are much uncertainty about the future realizations making necessary to incorporate some form of risk aversion in the optimization model. Several risk measures have been used for portfolio optimization [42], the Conditional Value at Risk (CVaR) has recently become widely used because it is a coherent risk measure [61,62] [63] [51].

It is very important to use a time consistency model with a clear interpretation of the objective function. To overcome skepticism and earn the investors' trust, it is necessary that they understand what is the main objective of the optimized portfolio. Time consistency is also a concern, since inconsistency may not take risk aversion into account. Therefore, we are going to adopt a recursive model based on convex combination of the expected value and CVaR, as it ensures temporal consistency and can be interpreted as certainty equivalent of the fund.

Section 4.2 describes a brief review of the SDDP with risk neutral and risk averse proposals, in Section 4.3 we present the SDDP methodology modified to asset allocation, with different assumptions, our proposal the H2SDDP and the ambiguity aversion approach. Finally, Section 4.4 brings the experiments in portfolio selection to analyze how the methods will perform.

\subsection{Stochastic Dynamic Dual Programming}

Stochastic Dynamic Dual Programming (SDDP) is an extension of the Benders decomposition [64] that overcomes the curse of dimensionality, common in multistage stochastic problems, by creating for each stage an approximation of the cost-to-go function using a set of linear functions (cutting planes) [48]. 
In the literature we can find other multistage stochastic programming methods that also uses sample average approximation [50] [49], all these methods successively sample the distribution, constructing better approximations of the cost-to-go function. What distinguishes SDDP from the other methods is how the cutting planes are evaluated, using the dual solution, making possible to share cuts between solution of the same stage.

SDDP, and most of the sampling-based methods, assumes stagewise independence to avoid having multiple cuts by stage. Considering stagewise independence, the cutting planes can be shared between solutions from the same stage. Thus only one approximation of the cost-to-go function has to be constructed at each stage.

The SDDP algorithm is divide into two main parts, a simulation forward in time and a backward in time recursion. It generates trials solutions $x_{t j}$ in forward step and in backward step uses this solutions to compute the cutting planes for cost-to-go functions.

More specifically, forward step sample $m \in\{1, \ldots, M\}$ scenarios to construct trial solutions $x_{t}^{m}$ chosen from $N_{t}$ random realizations for every stage $t$. For each of these scenarios and stages it is evaluated the optimal solution using the current approximation of the cost-to-go functions. This procedure has this name because it goes forward in time taking samples from 1 to $T$ stages.

Backward step goes in the opposite time direction, from $T$ to 1, taking advantage of subsequent evaluated cuts. In backward step, the trial solutions obtained in forward step are used as the solution for previous stage $\mathbf{x}_{t-1}$. For each stage and sample it solves a problem to find the optimal value $\mathbf{x}_{t}$. These results are used to calculate the cutting plane of the stages and then added to the corresponding stage's set of cutting planes.

\section{(a) Risk Neutral SDDP}

The following section is dedicated to present the dynamic programming equations for the original SDDP, keeping in mind the assumption of stagewise independence. The notation adopted herein is similar to that of Shapiro in [60]. SDDP constructs a Sample Average Approximation (SAA) of the "true" problem by sampling scenarios, typically using Monte Carlo. The generic risk neutral formulation for multistage stochastic problems is

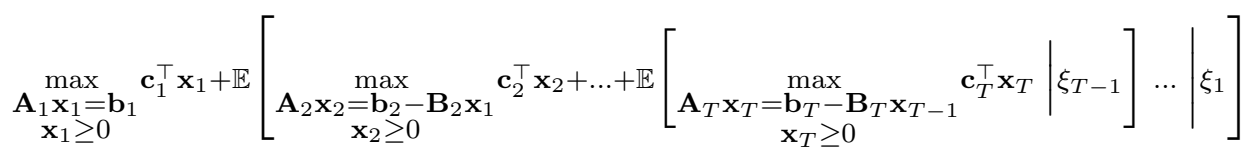


The number of stages is $T$ and the number of conditional discrete realization in each stages is $N_{t}$. In this problem, a realization is

$$
\xi_{t}(s)=\left\{\mathbf{c}_{t}(s), \mathbf{A}_{t}(s), \mathbf{B}_{t}(s), \mathbf{b}_{t}(s)\right\}
$$

To reduce notation, in this formula and throughout the text $\xi_{t}(s)$ has been simplify, leaving $\xi_{t s}=\left\{\mathbf{c}_{t s}, \mathbf{A}_{t s}, \mathbf{B}_{t s}, \mathbf{b}_{t s}\right\}$. Assuming independent samples with $\xi_{t} \in\left\{\xi_{t, 1}, \ldots, \xi_{t, N_{t}}\right\}, \forall t \in\{2, \ldots, T\}$, that means $\xi_{t}$ does not depend on $\left\{\xi_{1}, \ldots, \xi_{t-1}\right\}$ and that the cost-to-go functions $Q_{t+1}\left(\mathbf{x}_{t}, \xi_{t+1}\right)$ is independent from past data. The multistage stochastic dynamic programming equation is

$$
\begin{array}{cc}
Q_{t}\left(\mathbf{x}_{t-1}, \xi_{t s}\right)=\max _{\mathbf{x}_{t}} \mathbf{c}_{t s}^{\top} \mathbf{x}_{t}+\mathcal{Q}_{t+1}\left(\mathbf{x}_{t}\right) & \\
\text { s.t. } & \mathbf{A}_{t s} \mathbf{x}_{t}=\mathbf{b}_{t s}-\mathbf{B}_{t s} \mathbf{x}_{t-1} \\
\mathbf{x}_{t} \geq 0 & : \boldsymbol{\pi}_{t s}
\end{array}
$$

where $\boldsymbol{\pi}_{t s}$ is the vector of dual variables.

In this case, the cost-to-go function is

$$
\mathcal{Q}_{t+1}\left(\mathbf{x}_{t}\right)=\frac{1}{N_{t}} \sum_{s=1}^{N_{t}} Q_{t+1}\left(\mathbf{x}_{t}, \xi_{t+1, s}\right), \forall t \in \mathcal{H}
$$

In the last stage $Q_{T}(\cdot) \equiv 0$. The cost-to-go function $\mathcal{Q}_{t+1}\left(\mathbf{x}_{t}\right)$ shall be approximated by a set of cutting planes $\mathcal{I}_{t}$

$$
\mathfrak{Q}_{t}\left(\mathbf{x}_{t-1}\right)=\max _{l \in \mathcal{I}_{t}}\left\{\widetilde{\mathcal{Q}}_{t}\left(\mathbf{x}_{t-1, l}\right)+\widetilde{\mathbf{g}}_{t l}^{\top}\left(\mathbf{x}_{t-1}-\mathbf{x}_{t-1, l}\right)\right\}, \forall t \in \mathcal{H}
$$

where $\widetilde{\mathcal{Q}}_{t}\left(\mathbf{x}_{t-1, l}\right)=\frac{1}{N_{t}} \sum_{s=1}^{N_{t}} \widetilde{Q}_{t}\left(\mathbf{x}_{t-1, l}, \xi_{t s}\right)$ and $\widetilde{\mathbf{g}}_{t l}=-\frac{1}{N_{t}} \sum_{s=1}^{N_{t}} \boldsymbol{\pi}_{t s l} \mathbf{B}_{t s}$.

Using the dynamic programming equations, the SDDP algorithm can be explained in further detail. In forward step, it uses $\widetilde{Q}_{t+1}\left(\mathbf{x}_{t}\right)$ with current collections of cutting planes, to find the trials $\left\{\mathbf{x}_{1}^{m}, \cdots, \mathbf{x}_{T}^{m}\right\}$ for backward step. Backward part goes from $T$ to 1 , using the trial solutions as $\mathbf{x}_{t-1}$ to add new cuts by evaluating $\widetilde{\mathcal{Q}}_{t}\left(\mathbf{x}_{t-1, l}\right)$ and $\widetilde{\mathbf{g}}_{t l}$. The number of scenarios sampled in forward will determine how many cuts will be added for iteration.

An estimate of an lower bound is evaluated during the forward procedure by multiplying the trial solutions by the cost. The upper bound is obtained by solving the first stages' problem, using the sets of cutting planes $\mathfrak{Q}_{2}(\cdot)$.

\section{(b) Time Consistent Risk Averse Model}

This section presents the formulation for the risk averse model for multistage asset allocation problems, using CVaR as risk measure and assuming independence between stages. It is important to notice that the definition 
of Value-at-risk for gain distributions [63] differs from the original [65], when the domain is the final net worth(gain). To differentiate both definitions, we will call the Value-at-risk for gain distributions VaR and the traditional Valueat-risk for net worth VaR, analogously for CVaR and CVaR. As this section will cover gain distributions, it will use CVaR, as in [63].

\section{Conditional value at risk}

Standard deviation is the most commonly used measure of dispersion for asset allocation, however, there are other popular risk measures, such as Absolute Deviation and Mean-Absolute Moment, as well as downside measures like semivariance, Value at Risk (VaR) and Conditional Value at Risk (CVaR), [1]. While dispersion measures attribute an equal weight to the deviations below and above the mean, for downside risk measure the most important part is on realizations worse than the portfolio mean return. The downside risk measure suggests that the left-hand side of a return distribution gives information about risk, while the right-hand side contains investment opportunities [66].

One of the preferred downside measures is VaR, vastly used by financial institutions to report on the exposure of their portfolios to risk. VaR is the maximum portfolio loss within a certain confidence level $\alpha$, normally $95 \%$ or $99 \%{ }^{1}$. The popularity of this measure is due to its simple interpretation as the maximum reasonable loss for a given portfolio. Formally, for a random variable $Z \mathrm{VaR}$ is defined $\mathrm{as}^{2}$

$$
\operatorname{VaR}_{\alpha}(Z)=\inf \{l: \mathbb{P}(\{\omega \in \Omega \mid-Z(\omega) \geq l\}) \leq(1-\alpha)\}
$$

Despite the popularity of VaR, this risk measure has several undesirable properties. For one, it does not consider extreme events, changes on the tail distribution will not have any impact on VaR. It is not subadditive, and more diversification may lead to an increase in the portfolio risk, what also creates a non convex function, making it unattractive for optimization. These issues with VaR motivated the creation of a set of desired properties for risk measures, i.e. what properties they should have to become coherent risk measures [65].

Therefore, using $\mathcal{Z}$ as the set of possible gains, for a risk measure $\rho: \mathcal{Z} \rightarrow \overline{\mathbb{R}}$ to be coherent it must satisfy the following axioms (for $Z$ and $\left.Z^{\prime} \in \mathcal{Z}\right):$

1. Convexity, $\rho\left(l Z+(1-l) Z^{\prime}\right) \leq l \rho(Z)+(1-l) \rho\left(Z^{\prime}\right) \quad l \in[0,1]$;

\footnotetext{
${ }^{1}$ This definition differs from [63], where the $(1-\alpha)$ is the confidence level and $\alpha$ is a small number

${ }^{2}$ As we are using gains $W_{t}$, we will use the original definition of $\mathrm{VaR}$ [65] when the domain is gain and not loss.
} 
2. Monotonicity, if $Z \leq Z^{\prime}$ then $\rho(Z) \leq \rho\left(Z^{\prime}\right)$;

3. Translation equivalence, if $a \in \mathbb{R}$ then $\rho(Z+a)=\rho(Z)+a$;

4. Positive homogeneity, if $l>0$ then $\rho(l Z)=l \rho(Z)$.

It is common to find in the literature studies defining the subadditivity property instead of the convexity, because subadditivity and positive homogeneity are sufficient to ensure that $\rho(\cdot)$ is convex.

Over the last decade, a special effort has been made to use and find coherent risk measures, CVaR being one example of solution found for this purpose. Also known as expected tail loss (ETL), it is the conditional expectation of losses beyond the VaR, and the mean of the distribution tail on the right side of the VaR. It can also be simply explained as the expected loss when the VaR is exceeded. CVaR is defined for a loss distribution(-Z)

$$
\operatorname{CVaR}_{\alpha}(Z)=\operatorname{VaR}_{\alpha}(Z)+(1-\alpha)^{-1} \mathbb{E}\left[-Z-\operatorname{VaR}_{\alpha}(Z)\right]_{+}
$$

Initially proposed by Rockafellar and Uryasev [62] for portfolio optimization, the advantages brought by the CVaR, often proposed as a substitute for the VaR, have led to many studies and applications [61,63,67]. It was lately used by Shapiro [63] in conjunction with SDDP to propose and solve a risk aversion multistage stochastic problem.

In definition (4.7), it is important to note that CVaR is an upper bound for VaR, i.e. CVaR is always equal or greater than VaR. As a result, one solution with constraint $C V a R_{\alpha}(Z) \leq \kappa$ will remain feasible for $\operatorname{VaR}_{\alpha}(Z) \leq \kappa$. Figure 4.1, illustrating the concept of VaR and CVaR for loss distributions.

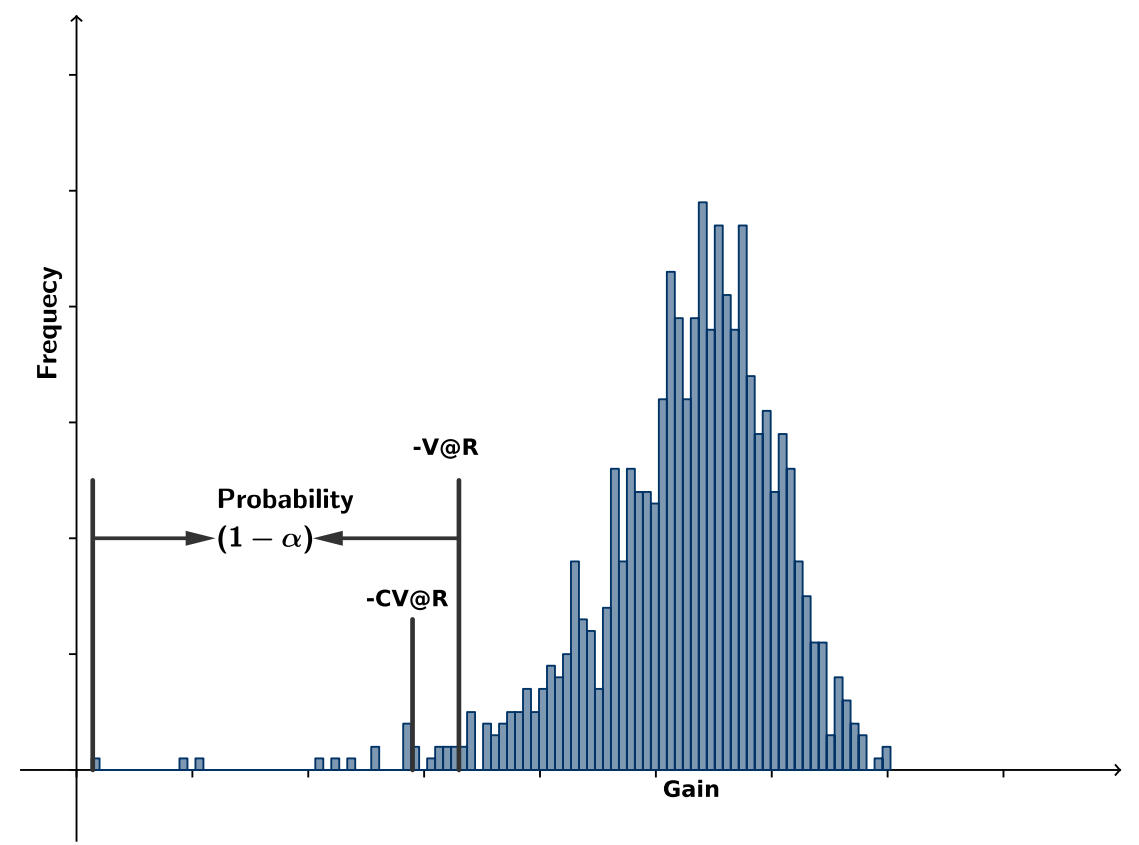

Figure 4.1: -CVaR and -VaR for gain distribution 
As a coherent risk measure, the CVaR should be an appropriate way to assess the risk. However, we want to stress the fact that if one chooses a dynamic model, time consistency should be a concern since an inconsistent policy may not consider aversion risk [68].

In a maximization problem it can also be used an alternative definition using -CVaR to evaluate the equation above, as follows

$$
-\operatorname{CVaR}_{\alpha}(Z)=\sup _{u \in \mathbb{R}}\left\{u-\frac{\mathbb{E}\left[(Z-u)^{-}\right]}{1-\alpha}\right\}
$$

where $x^{-}=-\min (x, 0)$.

\section{(c) Stopping Criteria}

For general deterministic problems, as well as for deterministic Benders decomposition, the optimal solution is achieved when the lower bound and the upper bound are the same. However, the SDDP problem is SAA of the "true" problem, thus the lower and upper bound are an approximation of the actual value for the real "true" problem, and one has to consider the confidence interval of this values to compare them.

Although the finite convergence of SDDP and other sampling-based methods has been proven $[63,69]$, the computational time required for the algorithm to actually converge could be considerable. The traditional stopping criterion proposed by Pereira e Pinto [53] consists in stopping SDDP when the upper bound get into the lower bound's confidence interval. As exposed by Shapiro 2011 [63], this criterion does not seem to make much sense and it would be more logical if the algorithm stopped when the gap between the upper limit of the lower bound and the upper bound became small enough $(<\epsilon)$. However, this gap can be very large and normally does not get narrow enough within a reasonable computation time [70].

There has been debate around what should be the stopping criteria of SDDP. Several other different stopping criteria have been proposed in the literature, but to this date none has been widely accepted as being the best one. Additionally, finding the lower bound for the multistage stochastic maximization problems that uses nested CVaR [63] is not easy. In spite of some promising attempts [71], as seen above, there is no guarantee that by using a stopping criteria with lower bound the solution of SDDP will converge. Actually, in practical problems, achieving convergence is very time-consuming, thus most of the time the SDDP has to be stopped before finding the optimal solution. Further debate on the convergence of SDDP can be found in $[63,72]$. Despite all the discussion around the convergence of SDDP, the most 
straightforward method to stop SDDP with nested CVaR is the stabilization of the upper bound. As pointed out by Shapiro [63], the stabilization of the upper bound does not mean that SDDP is close to the optimal solution. However, during this thesis, when stabilization was achieved, even by changing the number of trials and leaving the algorithm running for many days, the upper bound could not be changed. Thus, given the fact that none of the methods has guaranteed convergence, we think that the stabilization of the upper bound represents the better trade off between the computational time and proximity to the optimal solution.

\section{(d) Sampling Scenarios}

The idea to represent the random process through samples was used by different authors over the years. Sample Average Approximation (SAA) consists in representing a distribution by random realizations sampled from "true" distributions. Therefore, the "true" optimization problem is replaced by its SAA version. The method traditionally used in SAA is the Monte Carlo, that randomly selects independent and identically distributed (i.i.d.) samples, all realizations having an equal probability of being selected.

In practical situations, sampling the scenarios with Monte Carlo requires a large number of samples in order to keep deviations small. Nonetheless, there are techniques that can be used to reduce the variance, like Importance Sampling and Latin Hypercube Sampling.

Importance sampling having been created to be a variance reduction technique [73], but is also particularly useful when it is impossible to sample from the real distribution, it allows to study and analyze a distribution using samples taken from another distribution.

Assuming that the likelihood ratio function $\frac{f\left(x_{i}\right)}{g\left(x_{i}\right)}$ is well defined, if there is some $z \in \mathbb{R}^{d}$ where $f(z)=0$, then $g(x)=0$, the expected value of a random variable $X \sim h$ can be calculated as

$$
\mathbb{E}(h(X))=\int_{\mathcal{D}} h(x) f(x) d x=\int_{\mathcal{D}} \frac{h(x) f(x)}{g(x)} g(x) d x=\mathbb{E}_{g}\left(\frac{h(X) f(X)}{g(X)}\right)
$$

For a given set of i.i.d. samples $x_{i}$ drawn according to a probability density $g(X)$ the importance sampling estimator of $\mu=\mathbb{E}_{g}(h(X))$ is

$$
\hat{\mu}_{g}=\frac{1}{n} \sum_{i=1}^{n} \frac{h\left(x_{i}\right) f\left(x_{i}\right)}{g\left(x_{i}\right)}, x_{i} \sim g
$$

The importance sampling estimator $\hat{\mu}_{g}$ is an unbiased estimator of $\mu$. By sampling from distribution probability density function $g(\cdot)$ and making an 
adjustment using the likelihood ratio $\frac{f\left(x_{i}\right)}{g\left(x_{i}\right)}$, it is also possible to obtain samples from the original or nominal distribution $h$.

This approach in SDDP may be used, for example, for a cost-to-go function $Q_{t}\left(\mathbf{x}_{t-1}, \xi_{t s}\right)$, while an importance sampling estimator can be evaluated given realizations different form $\xi$. However, as indicated by Shapiro in [60], the importance sampling method has some issues, it is very sensitive to the choice of p.d.f., for example, and its instability is notorious, even for small perturbations in $g(\cdot)$.

Latin Hypercube Sampling, another variance reduction technique, uses probability dense inverse function to sample the distribution more evenly. Initially proposed by Mckay et al. 1979 [74], it is a stratified sampling method, a K-dimensional extension of Latin square sampling. Latin Hypercube Sampling divide the p.d.f. in intervals with the same probability and collect one sample from each interval. Controlling how random samples are generated, the method spreads the samples more evenly throughout the distribution.

It is possible to observe on Figure 4.2 how Latin Hypercube Sampling method performs on two uniform distributions $X \sim U(0,1)$ and $Y \sim U(0,1)$.

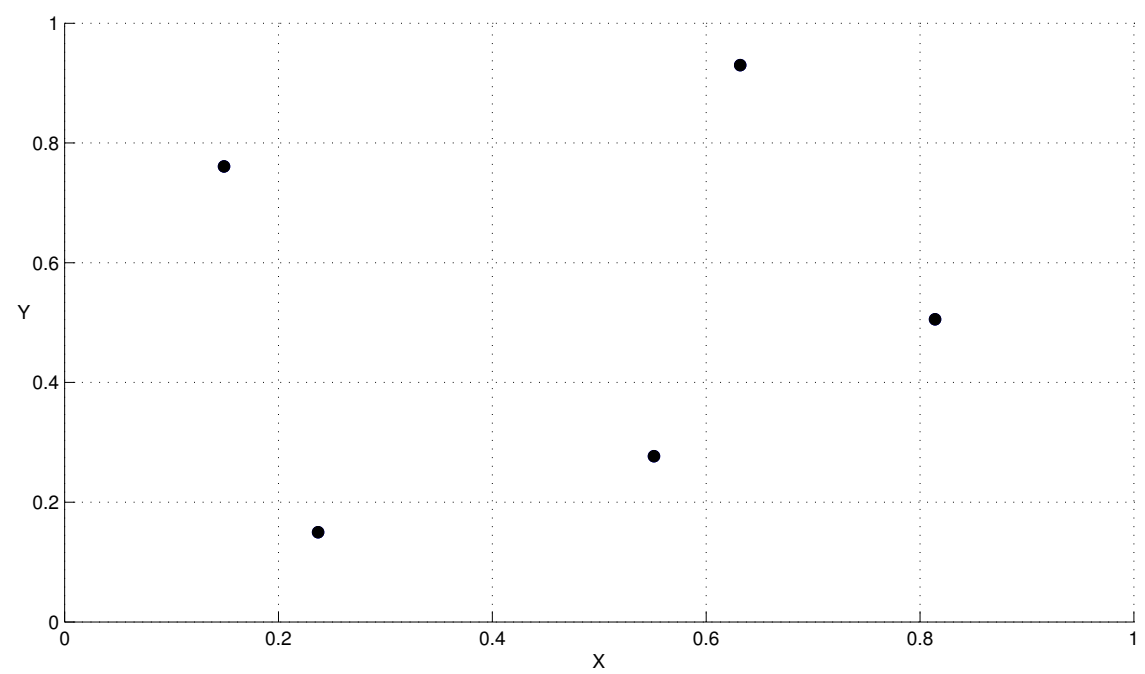

Figure 4.2: Example of Latin Hypercube Sampling for uniform distribution with two dimensions

To illustrate how Latin Hypercube Sampling and Monte Carlo behave, two examples were designed, which can be seen in Figure 4.3 and Figure 4.4. For the first example, five hundred iterations were performed, sampling 30 points of normal distribution $N \sim(0,1)$ by using Monte Carlo and also Latin Hypercube Sampling. The resulting figures present the estimated normal distribution for each one of the five hundred iterations. 


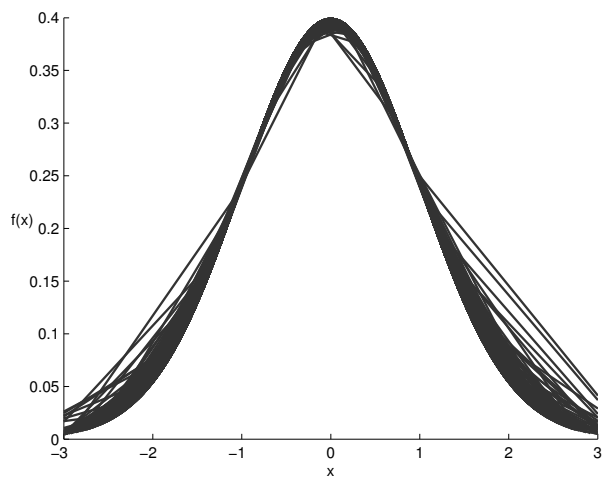

Figure 4.3: Sampling with Monte Carlo

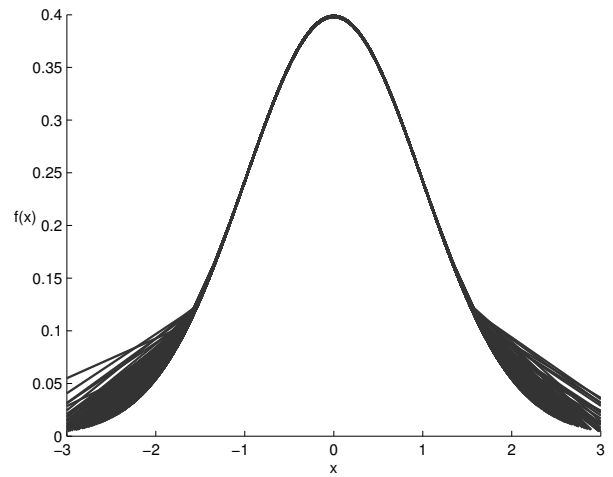

Figure 4.4: Sampling with Latin Hypercube Sampling

On the figure it is clear that the variance of the Monte Carlo is larger than the Latin Hypercube Sampling. Latin Hypercube Sampling also requires a reasonable number of examples, mainly to better represent the distribution tails. The intervals on the tails are bigger, as we can see in Figure 4.5, thus more samples would be necessary to better represent this part of the distribution.

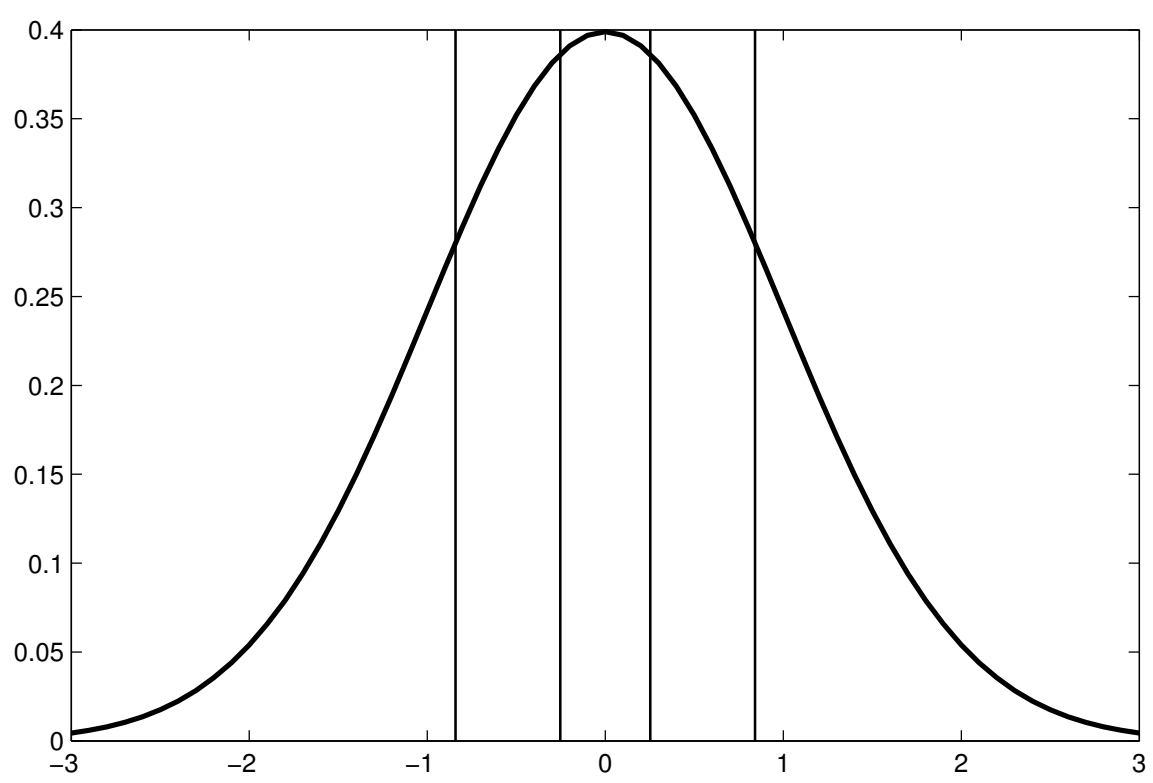

Figure 4.5: LHS interval for a normal distribution $N \sim(0,1)$

\subsection{Stochastic Dynamic Programming for Asset Allocation}

In this section we will explain how to adapt SDDP for multistage asset allocation. First, we will begin with the risk-neutral formulation and risk averse 
formulation, followed by the time independent model, transactional costs with SDDP and, finally, by our proposal to treat temporal dependence.

Supposing an investor wishes to construct a portfolio dividing a given amount of cash among the assets $\left\{x_{t i}, \forall i \in \mathcal{A}\right\}$ each of which has an uncertain return $r_{t i}(s)$ for time $t$. Each stage brings the need to reallocate the portfolio, considering the profits obtained in the previous stage $W_{t}=\left(\mathbb{1}+\mathbf{r}_{t}\right)^{\top} \mathbf{x}_{t-1}$. Considering an initial capital $W_{1}$, and not allowing a short sell (i.e. $\mathbf{x} \geq 0$ ), the risk-neutral portfolio multistage stochastic problem is stated as

$$
\max _{\substack{\mathbb{1}^{\top} \mathbf{x}_{1}=W_{1} \\ \mathbf{x}_{1} \geq 0}} \mathbb{E}\left[\max _{\substack{\mathbb{1}^{\top} \mathbf{x}_{2}=W_{2} \\ \mathbf{x}_{2} \geq 0}} \mathbb{E}\left[\max _{\substack{\mathbb{1}^{\top} \mathbf{x}_{t-1}=W_{T-2} \\ \mathbf{x}_{t-1} \geq 0}} \mathbb{E}\left[W_{T} \mid \overline{\mathbf{r}}_{[T-1]}\right] \ldots \mid \overline{\mathbf{r}}_{1}\right]\right]
$$

The realization of the investment made in $t$ is accounted for in the following stage $t+1$. The optimal solution for this risk-neutral formulation would be to allocate everything in the asset with the highest expected profit. However, it is a known fact that investing everything in a single asset is not very wise. Neglecting risk in the optimization problem can lead to big losses, especially in fall trends.

Using $\psi(\cdot)_{t}$ function it is possible to add risk aversion formulation for asset allocation

$$
\max _{\substack{\mathbb{1}^{\top} \mathbf{x}_{1}=W_{1} \\ \mathbf{x}_{1} \geq 0}} \psi_{1}\left[\max _{\substack{\mathbb{1}^{\top} \mathbf{x}_{2}=W_{2} \\ \mathbf{x}_{2} \geq 0}} \psi_{2}\left[\max _{\substack{\mathbb{1}^{\top} \mathbf{x}_{t-1}=W_{T-2} \\ \mathbf{x}_{t-1} \geq 0}} \psi_{T-2}\left[W_{T} \mid \overline{\mathbf{r}}_{[T-1]}\right] \ldots \mid \mathbf{r}_{1}\right]\right]
$$

The function $\psi(\cdot)_{t}$ is the convex combination combination of the expect value and the CVaR, as it is a maximization problem. Formally, the function $\psi_{t}(\cdot)$ is

$$
\psi_{t}\left(Z \mid \xi_{t}\right)=(1-\lambda) \mathbb{E}\left[Z \mid \xi_{t}\right]+\lambda\left(-\mathrm{CVaR}_{\alpha}\left[Z \mid \xi_{t}\right]\right)
$$

\section{(a) Myopic policy: No transaction costs and temporal in- dependence}

For the special case when the multistage portfolio selection does not have transactional cost and no temporal dependence the optimal solution can be easily found using a myopic policy. In the appendix A.2 it is presented the myopic prove for a coherent risk measure [60].

The myopic policy, that aims on maximizing one-step reward ignoring the impact on future rewards, is optimal. However, this policy is optimal in a very specify situation. In a more realist situation, with transactional costs, the myopic policy is not optimal. 


\section{(b) SDDP for asset allocation: Transaction costs and tem- poral independence}

In this section, the asset allocation problem will be formulated with $\mathbf{x}_{t}$ in the state space and risk aversion with CVaR, in order to be used in SDDP and to later include transactional costs. However, this model still disregards the temporal dependence.

According to Rudloff et al. [68], the nested CVaR is the certainty equivalent of the portfolio and, consequently, the worth of the portfolio. Therefore, for the multistage asset allocation problem the cost-to-go is actually the worth function. The dynamic programming equation can be defined using the risk averse multistage formulation for asset allocation (4.12) and the function $\psi(\cdot)(4.13)$. The worth function is

$$
\begin{aligned}
& Q_{t}\left(\mathbf{x}_{t-1}, \overline{\mathbf{r}}_{t}\right)=\max _{\mathbf{x}_{t}} \overline{\mathbf{r}}_{t}^{\top} \mathbf{x}_{t-1}+\mathcal{Q}_{t+1}\left(\mathbf{x}_{t}\right) \\
& \text { s.t. } \mathbb{1} \mathbf{x}_{t}=\left(\overline{\mathbf{r}}_{t}+\mathbb{1}\right)^{\top} \mathbf{x}_{t-1} \\
& \mathbf{x}_{t} \geq 0
\end{aligned}
$$

and the expected recourse function, in this case, the portfolio expected worth function is

$$
\begin{gathered}
\mathcal{Q}_{t+1}\left(\mathbf{x}_{t}\right)=(1-\lambda) \frac{1}{N_{t}} \sum_{s=1}^{N_{t}} Q_{t+1}\left(\mathbf{x}_{t}, \mathbf{r}_{t+1}(s)\right)+ \\
\lambda\left(Q_{t+1}\left(\mathbf{x}_{t}, \mathbf{r}_{t+1}(\tau)\right)-\frac{1}{(1-\alpha) N_{t}} \sum_{s=1}^{N_{t}}\left[Q_{t+1}\left(\mathbf{x}_{t}, \mathbf{r}_{t+1}(s)\right)-Q_{t+1}\left(\mathbf{x}_{t}, \mathbf{r}_{t+1}(\tau)\right)\right]^{-}\right)
\end{gathered}
$$

Is important to emphasize that the $Q_{t+1}\left(\mathbf{x}_{t}, \mathbf{r}_{t+1}(\tau)\right)$ is the $-\mathrm{VaR}$ of $\left\{Q_{t+1}\left(\mathbf{x}_{t}, \mathbf{r}_{t+1}(1)\right), \ldots, Q_{t+1}\left(\mathbf{x}_{t}, \mathbf{r}_{t+1}\left(N_{t}\right)\right)\right\}$, i.e the right or positive $\alpha$ quantile. Herein the -CVaR, and consequently -VaR, will be evaluated using the dual representation of the CVaR that is a fractional knapsack problem [61,65].

$$
\begin{array}{cc}
-C \operatorname{VaR}(X)=\min _{v_{s}} \sum_{s=1}^{N_{t}} Q_{t+1}\left(\mathbf{x}_{t}, \mathbf{r}_{t+1}(s)\right) \times v_{s} & \\
\text { s.t. } \sum_{s=1}^{N_{t}} v_{s}=1 & \\
v_{s} \leq \frac{1}{(1-\alpha) N_{t}} & \forall s \in\left\{1, \ldots, N_{t}\right\} \\
v_{s} \geq 0 & \forall s \in\left\{1, \ldots, N_{t}\right\}
\end{array}
$$

To determine the cut, it is necessary to evaluate the subgradient of the 
worth function $\mathcal{Q}_{t}\left(\mathbf{x}_{t-1}\right)$, it was used -CVaR. With $\gamma_{t s}$ being the subgradient of $\mathcal{Q}_{t}\left(\mathbf{x}_{t-1}^{*}\right)$ at point $\mathbf{x}_{t-1}^{*}$, the subgradient of the expected recourse function is

$$
\mathbf{g}_{t}=\nabla \mathcal{Q}_{t}\left(\mathbf{x}_{t-1}^{*}\right)=(1-\lambda) \frac{1}{N_{t}} \sum_{s=1}^{N_{t}} \gamma_{t s}+\lambda\left(\gamma_{t \tau}-\sum_{s=1}^{N_{t}} \boldsymbol{\eta}_{t s}\right)
$$

where using the weights $v_{s}$ of (4.16) we have $\boldsymbol{\eta}_{t s} \forall s \in\left\{1, \ldots, N_{t}\right\}$

$$
\boldsymbol{\eta}_{t s}= \begin{cases}0 & \text { if } Q_{t}\left(\mathbf{x}_{t-1}, \mathbf{r}_{t}(s)\right)>Q_{t}\left(\mathbf{x}_{t-1}, \mathbf{r}_{t}(\tau)\right) \\ -\left(\boldsymbol{\gamma}_{t+1, s}-\boldsymbol{\gamma}_{t+1, \tau}\right) v_{s} & \text { if } Q_{t}\left(\mathbf{x}_{t-1}, \mathbf{r}_{t}(s)\right) \leq Q_{t}\left(\mathbf{x}_{t-1}, \mathbf{r}_{t}(\tau)\right)\end{cases}
$$

and the subgradient of $Q_{t}\left(\mathbf{x}_{t}\right)$ changes to

$$
\gamma_{t}=\mathbf{r}_{t}+\left(\mathbb{1}+\mathbf{r}_{t}\right)^{\top} \boldsymbol{\pi}^{*}
$$

It must be kept in mind that traditional SDDP is a minimization problem, however, this work maximizes the objective function, thus the solution of $Q_{t}\left(\mathbf{x}_{t-1}, \overline{\mathbf{r}}_{t}\right)$ is an upper bound to the model presented here.

\section{Transactional costs}

Most of the early asset allocation methods did not consider the transactional costs on purchases or sales of assets. When the costs of each transaction are neglected, the gain apparently obtained can be misleading. Depending on the frequency of the transactions and costs, an estimated positive return could, in reality, became loss. Moreover, costs stimulate the maintenance of the selected portfolio in the previous period, because it is an explicit regularization of portfolio movements. It is a L1 regularization [75], since the sum of the absolute differences, the changes on the portfolio, is penalized on the objective function. It will be seen later in the experiments section this behavior in practice.

Introducing $d_{t i}^{+}$and $d_{t i}^{-}$to represent how much from each asset $i$ is bought and sold. Also, the variable $c$ that is the proportional cost, selling will bring an income $(1-c) d_{t i}^{-}$, and buying will mean a cost $(1+c) d_{t i}^{+}$to current wealth $\left(\mathbb{1}+\overline{\mathbf{r}}_{t}\right)^{\top} \mathbf{x}_{t-1}$. The problem with transactional cost can be formulated as

$$
\begin{array}{cc}
Q_{t}\left(\mathbf{x}_{t-1}, \overline{\mathbf{r}}_{t}\right)= & \max _{\mathbf{x}_{t}, \mathbf{d}_{t}^{+}, \mathbf{d}_{t}^{-}} \\
\text {s. t. } & \overline{\mathbf{r}}_{t}^{\top} \mathbf{x}_{t-1}-\sum_{i \in A} c\left(d_{t i}^{+}+d_{t i}^{-}\right)+\mathcal{Q}_{t+1}\left(\mathbf{x}_{t}\right) \\
& x_{t i}-c \sum_{i \in A}\left(d_{t i}^{+}+d_{t i}^{-}\right)=\left(\mathbb{1}+\overline{\mathbf{r}}_{t}\right)^{\top} \mathbf{x}_{t-1}^{-}=\left(1+\bar{r}_{t i}\right) x_{t-1, i}, \forall i \in \mathcal{A} \backslash\{1\} \\
& \mathbf{d}_{t}^{+}, \mathbf{d}_{t}^{-}, \mathbf{x}_{t} \geq 0
\end{array}
$$


Notice that there is no transactional cost in the purchase or sale of riskfree asset. Furthermore, in this model the complementarity of the simultaneous purchase and sale of a given asset is guaranteed, i.e. $d_{t i}^{+} d_{t i}^{-}=0$. If there were simultaneous purchase and sale operations of an asset, that could not be the best solution as it would be possible to obtain a better return without interfering with $x_{t i}$.

The subgradient of the worth function for SDDP also changes. In this context there are additionally $A$ dual variables, represented by the vector $\boldsymbol{\pi}_{2} \in \mathbb{R}^{A}$ associated with constraint (4.22), then the subgradient of $Q_{t}\left(\mathbf{x}_{t}\right)$ become

$$
\boldsymbol{\gamma}_{t}=\overline{\mathbf{r}}_{t}+\left(\mathbb{1}+\overline{\mathbf{r}}_{t}\right)^{\top} \boldsymbol{\pi}_{1}^{*}+\left(\mathbb{1}+\overline{\mathbf{r}}_{t}\right)^{\top} \boldsymbol{\pi}_{2}^{*}
$$

with $\boldsymbol{\pi}_{1}$ representing the dual variables of constraint (4.21). Furthermore, the optimal solution of this new problem cannot be found using myopic policy, now it cannot ignore future implications caused by the current decision.

\section{(c) H2SDDP for allocation: Transaction costs and temporal dependence}

In previous sections it was shown that when the multistage asset allocation problem has no time dependency the optimal solution can be achieved using a myopic policy. When transactional costs are added to the model this myopic optimal behavior does not hold, under these circumstances the traditional SDDP can be used to find the optimal solution. However, the stagewise assumption of traditional SDDP is an issue when modeling temporal dependence problems. In this section we will discuss some approaches to model time dependency and how to modify SDDP for this purpose.

In many applications a more general problem has to be considered, when there is time dependency between the stages. The worth function becomes dependent on the historical achievements of the random variable $\mathcal{Q}_{t+1}\left(\mathbf{x}_{t}, \overline{\mathbf{r}}_{[t]}\right)$. Thus, we have a worth function (future cost function on original SDDP) for each node in the stochastic tree, and therefore is so common to use L-shaped methods. The hydrothermal scheduling is one of the problems that typically has time dependency. This dependence usually occurs in inflows, on the righthand side of the problem. The inflows are modeled as an $\mathrm{AR}(1)$ autoregressive of order one, that is

$$
\mathbf{b}_{t}=\phi_{t} \mathbf{b}_{t-1}+\varepsilon_{t}
$$

Changing the stochastic process to $\xi_{t}=\left(\mathbf{c}_{t}, \mathbf{B}_{t}, \mathbf{A}_{t}, \boldsymbol{\varepsilon}_{t}\right)$ and increasing the state space to $\left(\mathbf{x}_{t}, \mathbf{b}_{t}\right)$, we can define the generic problem as well as solving it using the similar SDDP method used by independent case, problem (4.3), 
with the cost-to-go function as

$$
\begin{aligned}
Q\left(\mathbf{x}_{t-1}, \mathbf{b}_{t-1}, \xi_{t}\right)=\max _{\mathbf{x}_{t}} & \mathbf{c}_{t}^{\top} \mathbf{x}_{t}+\mathcal{Q}_{t+1}\left(\mathbf{x}_{t}\right) \\
\text { s. t. } & \mathbf{A}_{t} \mathbf{x}_{t}=-\mathbf{B}_{t} \mathbf{x}_{t-1}+\boldsymbol{\phi}_{t} \mathbf{b}_{t-1}+\boldsymbol{\varepsilon}_{t} \\
& \mathbf{x}_{t} \geq 0
\end{aligned}
$$

Furthermore, in hydrothermal scheduling there are situations where the inflows are best mapped as nonlinear functions, for example, as a Markov process. There are some advantages in mapping the time dependency as a Markov process, in addition to encapsulate all the historical information it also incorporate autocorrelated dependency, this is extremely important since seasonality is present in the inflows data [76].

Using Markovian process to represent the inflows probability distribution, it is also possible to preserve stagewise independence between cost-to-go functions. This approach was first develop in [54], and later adapted in [55] for the New Zealand electricity system and similar methodolodgy was applied to Energy contracts management of Liquefied Natural Gas in [77]. All these approaches use deterministic states and has dependency on the right-hand side.

\section{H2SDDP}

Undoubtedly, the methodology developed by Pereira and Pinto [48], SDDP, has great advantages, however, the assumption of stagewise independence is not realistic for some problems. When dealing with with temporal dependence, the methodology must go through some adaptation in order to be able to solve this type of problem.

In the alternative L-shaped method, there is one worth function for every node of the scenario tree, each node has a unique history and the worth function depends on the historical return. Therefore, this technique has greater computational complexity since the expected worth function has to consider the whole previous realization of the scenario.

The solution proposed here for multistage asset allocation is the Hidden States Stochastic Dynamic Programming (H2SDDP), that can be described as a combination of SDDP and L-shaped procedures. In the H2SDDP method, the market is classified and separated by states, so only the probability of each state depends on the past returns. The method can be seen as a generalization of SDDP and L-Shaped, if the number of states is equal to the number of nodes in the stochastic tree, the model will behave like L-Shaped; otherwise, if the number of states is one, it will act similarly to SDDP. 
The foremost difference between the three methods (SDDP, L-Shaped and H2SDDP) is the trade-off between how historical information is considered in each node of the stochastic tree and the computational complexity involved. With H2SDDP method we expected to achieve the best compromise in this regard, as it creates a small increase in complexity while being able to deal with problems with temporal dependence within a reasonable computational time.

In this work the temporal dependence will be expressed as states of hidden Markovian process and the asset return will be modeled as a multivariate mixture of Gaussians. There are other ways to separate financial time series in states, the HMM was chosen because it is more suitable for necessities involved in this circumstances. Indeed, the method is especially appropriate for this application because it can be used to estimate the states likelihood and to sample the return distributions given the states.

Many traditional financial applications used the normal distributions with stationary parameters to model the log-returns distributions. Nowadays, it is well known that this does not reproduce some important stylized facts or well known properties of the financial time series.

Fama [78] study analyses the behavior of stock market prices and presented some evidences that contradicts the assumption of normality. Granger and Ding [79] presented some of the stylized facts of properties for a variety of log return series. Rydén et al. [80] shown that modeling the financial time series as a Gaussian mixture according to the states of an unobserved Markov chain, the HMM model, reproduces most of the stylized facts for daily return series demonstrated by Granger and Ding [79].Thus, HMM is frequently used to model return series [80-83].

Figure 4.6 is an example of a simple a HMM for stock market, which uses, for the sake of simplicity, discrete emission distributions. The objective is to determine the market state Bull, Bear, Neutral given the return status of the current day stable, down, up. Observe that, given an emission, it is not possible to determine, with certainty, what is the state; and given a state and using the state emission distribution, we can obtain a realization. 


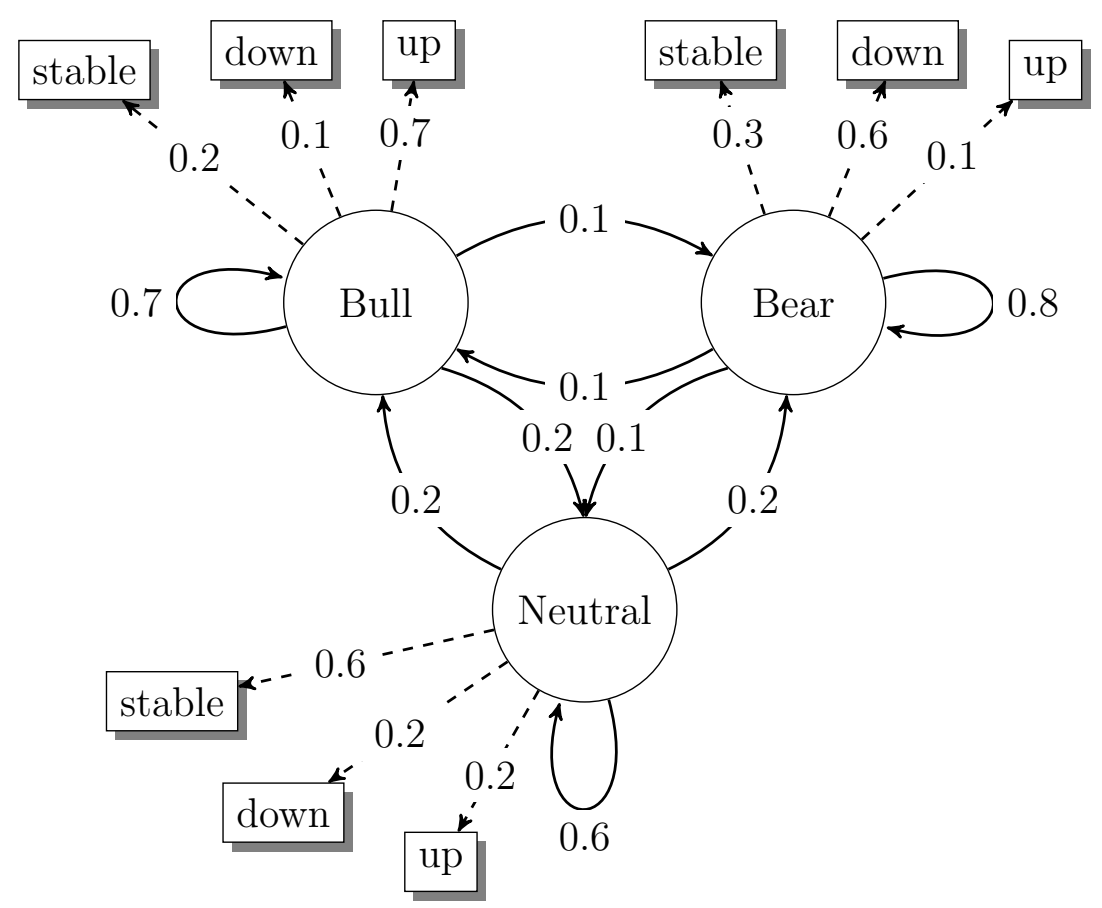

Figure 4.6: An example of HMM for stock market

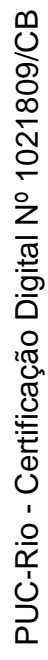

But in this application the return of each asset will depend on the state of the HMM, Figure 4.7 is an example on how return can be represented by HMM as a mixture of Gaussians.

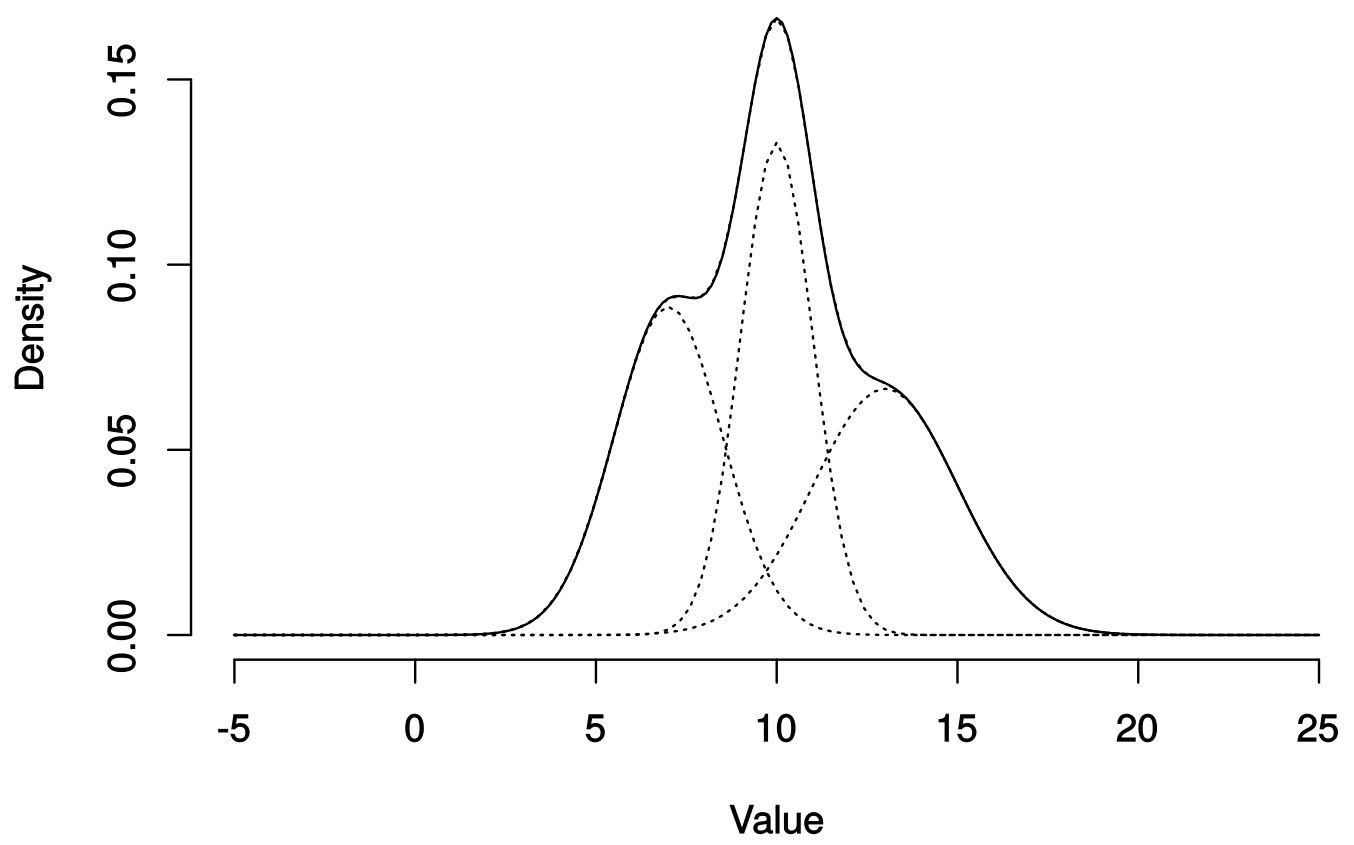

Figure 4.7: An example of a Gaussian mixture model.

Since temporal dependence of the return series will be modeled as a HMM, the SDDP's worth function will be separated by state, creating a stagewise independence. Therefore, there is only one worth function for each 
stage that depends on a specific market state. These functions do not depend on the previous returns, only the probability of the Markov states is conditional to historical returns. The downside of this approach is increasing computational effort, since it has more worth functions, one per state.

Let us first assume that at time $t$ the market state $k$ is known, then we solve the following problem

$$
\begin{gathered}
U_{t}\left(\mathbf{x}_{t} \mid k\right)=\psi_{t}\left[u_{t k}\left(\mathbf{x}_{t}, \mathbf{r}_{t+1}\right) \mid k\right] \\
u_{t k}\left(\mathbf{x}_{t}, \mathbf{r}_{t+1}(s)\right)=\mathbf{r}_{t+1}(s)^{\top} \mathbf{x}_{t}+\max _{\mathbf{x}_{t+1} \in X\left(\mathbf{x}_{t}, \mathbf{r}_{t+1}(s)\right)} \sum_{j=1}^{K} U_{t+1}\left(\mathbf{x}_{t+1} \mid j\right) P(j \mid k)
\end{gathered}
$$

where $U_{t}\left(\mathbf{x}_{t} \mid k\right)$ is the worth function given the state $k$ and the transition probability from state $k$ to $j$ is $P(j \mid k)$.

In practice, since the state $k$ is unknown, we need to solve problem (4.27) using the past observed returns $\mathbf{r}_{[t]}$ to estimate the states probabilities. To simulate the system and find the optimal policy we solve the following problem

$$
R_{t}^{P}\left(\mathbf{x}_{t-1}, \overline{\mathbf{r}}_{[t]}\right)=\overline{\mathbf{r}}_{t}^{\top} \mathbf{x}_{t-1}+\max _{\mathbf{x}_{t} \in X\left(\mathbf{x}_{t-1}, \overline{\mathbf{r}}_{t}\right)} \sum_{k=1}^{K} U_{t}\left(\mathbf{x}_{t} \mid k\right) P\left(k \mid \overline{\mathbf{r}}_{[t]}\right)
$$

where $P\left(k \mid \overline{\mathbf{r}}_{[t]}\right)$ is the likelihood of the state $k$ estimated by the forwardbackward algorithm [84] given the past returns $\mathbf{r}_{[t]}$.

The worth function $\mathcal{Q}_{t+1}\left(\mathbf{x}_{t}\right)$ is approximated by a set of cutting planes $\mathcal{I}_{t}$

$$
\mathfrak{U}_{t}\left(\mathbf{x}_{t} \mid k\right)=\max _{l \in \mathcal{I}_{t}}\left\{\widetilde{u}_{t k}\left(\mathbf{x}_{t-1, l}\right)+\widetilde{\mathbf{g}}_{t l}^{\top}\left(\mathbf{x}_{t-1}-\mathbf{x}_{t-1, l}\right)\right\}, \forall t \in \mathcal{H}
$$

where $\widetilde{u}_{t k}\left(\mathbf{x}_{t-1, l}\right)=\frac{1}{N_{t}} \sum_{s=1}^{N_{t}} \widetilde{u}_{t k}\left(\mathbf{x}_{t-1, l}, \mathbf{r}_{t}(s)\right)$ and $\widetilde{\mathbf{g}}_{t l}$ analogously to equation 4.17 with the subgradient equal to 4.24 .

To solve the problem (4.28), it is necessary to know each state's probability, in order to weigh the worth function of the next stage. Our approach with HMM uses past returns to estimate the present probability of each market state in forward step. With the states' probabilities, the objective function can be evaluate by combining the state probability with the respective worth function. Also, the states' probabilities of each stage determine the joint distributions that are used to establish the sequence of observed returns.

In backward step, each state emission distribution is considered and one or more cuts are added to each respective state. In other words, when realizations from a given state are used, the cut will be added to the set of cuts of that same state. Also, as in problem (4.28), the worth function is conditional on the state, meaning there will be $K$ functions per stage. Using the dynamic programming equations above, the models used in forward and 
backward steps are (4.30) and (4.31), respectively, with transactional costs.

$$
\begin{aligned}
\overline{\mathbf{x}}_{t}=\arg \max _{\mathbf{x}_{t}, \mathbf{d}_{t}^{+}, \mathbf{d}_{t}^{-}} & -\sum_{i} c\left(d_{t i}^{+}+d_{t i}^{-}\right)+\sum_{k=1}^{K} \mathfrak{U}_{t}\left(\mathbf{x}_{t} \mid k\right) P\left(k \mid \overline{\mathbf{r}}_{[1, t]}\right) \\
\text { s. t. } \mathbb{1}^{\top} \mathbf{x}_{t}+\sum_{i} c\left(d_{t i}^{+}+d_{t i}^{-}\right)=\left(\mathbb{1}+\overline{\mathbf{r}}_{t}\right)^{\top} \mathbf{x}_{t-1} & \\
& x_{t i}-d_{t i}^{+}+d_{t i}^{-}=\left(1+\bar{r}_{t i}\right) x_{t-1, i}, \\
& \mathbf{x}_{t}, \mathbf{d}_{t}^{+}, \mathbf{d}_{t}^{-} \geq 0 \\
u_{t k}\left(\mathbf{x}_{t}, \mathbf{r}_{t+1}(s)\right)= & \mathbf{r}_{t+1}(s)^{\top} \mathbf{x}_{t}+ \\
\max _{\mathbf{x}_{t+1}, \mathbf{d}_{t+1}^{+}, \mathbf{d}_{t+1}^{-}} & \sum_{i \in A} c\left(d_{t i}^{+}+d_{t i}^{-}\right)+\sum_{j=1}^{K} \mathfrak{U}_{t+2}\left(\mathbf{x}_{t+1} \mid j\right) P(j \mid k) \backslash\{1\} \\
\text { s. t. } \mathbb{1}^{\top} \mathbf{x}_{t+1}+\sum_{i \in A} c\left(d_{t i}^{+}+d_{t i}^{-}\right)=\left(\mathbb{1}+\mathbf{r}_{t+1}(s)\right)^{\top} \mathbf{x}_{t} & \\
& x_{t+1, i}-d_{t i}^{+}+d_{t i}^{-}=\left(1+r_{t+1, i}(s)\right) x_{t i}, \quad \forall i \in \mathcal{A} \backslash\{1\} \\
& \mathbf{x}_{t+1}, \mathbf{d}_{t+1}^{+}, \mathbf{d}_{t+1}^{-} \geq 0
\end{aligned}
$$

In the backward step the decision at $t$ are conditional to the state at time $t$ for every $t$ and for every state. In the forward step only the current first stage is treated differently, i.e., the state is assumed unknown. Note that, for the forward step the recourse or future decision are also conditioned to the states. Separating the worth functions for each state makes this approach an approximation for the SAA problem. The entire proposed algorithm for H2SDDP approach is presented below. 


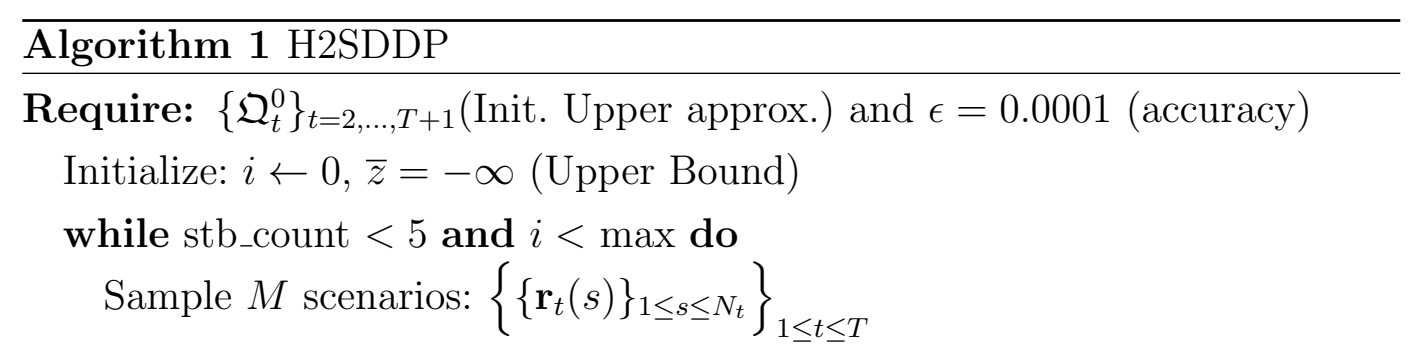

[Foward step]

for $m=1 \rightarrow M$ do

selects scenario $S_{m}=\left\{\overline{\mathbf{r}}_{0}, \ldots, \overline{\mathbf{r}}_{T}\right\}$

for $t=1 \rightarrow T$ do

$$
\overline{\mathbf{x}}_{t}^{m} \leftarrow \underset{\mathbf{x}_{t}}{\operatorname{argmax}}\left\{\begin{array}{l}
\sum_{k=1}^{K} \mathfrak{U}_{t}\left(\mathbf{x}_{t} \mid k\right) P\left(k \mid \overline{\mathbf{r}}_{[1, t]}\right): \\
\mathbb{1}^{\top} \mathbf{x}_{t}=\left(\mathbb{1}+\overline{\mathbf{r}}_{t}\right)^{\top} \overline{\mathbf{x}}_{t-1}^{m}, \mathbf{x}_{t} \geq 0
\end{array}\right\}
$$

end for

\section{end for}

\section{[Backward step]}

for $m=1 \rightarrow M, t=T \rightarrow 2$ do

$$
\text { for } k=1 \rightarrow K \text { do }
$$

for $s=1 \rightarrow N_{t}(k)$ do

$$
\left[\widetilde{u}_{t k}\left(\overline{\mathbf{x}}_{t-1}^{m}, \mathbf{r}_{t}(s)\right), \widetilde{\boldsymbol{\pi}}_{t}^{m}\left(\mathbf{r}_{t}(s)\right)\right] \leftarrow \max _{\mathbf{x}_{t}}\left\{\begin{array}{l}
\mathbf{r}_{t}(s)^{\top} \overline{\mathbf{x}}_{t-1}^{m}+\sum_{j=1}^{K} \mathfrak{U}_{t+1}\left(\mathbf{x}_{t} \mid j\right) P(j \mid k): \\
\mathbb{1}^{\top} \mathbf{x}_{t}=\left(\mathbb{1}+\mathbf{r}_{t}(s)\right)^{\top} \overline{\mathbf{x}}_{t-1}^{m}, \mathbf{x}_{t} \geq 0
\end{array}\right\}
$$

end for

$$
\begin{aligned}
& \widetilde{u}_{t k}\left(\overline{\mathbf{x}}_{t-1}^{m}\right) \leftarrow(1-\lambda) \frac{1}{N_{t}(k)} \sum_{s=1}^{N_{t}(k)}\left[\widetilde{u}_{t k}\left(\overline{\mathbf{x}}_{t-1}^{m}, \mathbf{r}_{t}(s)\right)\right]+ \\
& \quad \lambda\left\{\widetilde{u}_{t k}^{\tau}\left(\mathbf{x}_{t-1}, \mathbf{r}_{t}\right)-\frac{1}{(1-\alpha) N_{t}(k)} \sum_{s=1}^{N_{t}(k)}\left[\widetilde{u}_{t k}\left(\overline{\mathbf{x}}_{t-1}^{m}, \mathbf{r}_{t}(s)\right)-\right.\right. \\
& \left.\left.\widetilde{u}_{t k}^{\tau}\left(\mathbf{x}_{t-1}, \mathbf{r}_{t}\right)\right]^{-}\right\} \\
& \widetilde{\mathbf{g}}_{t}^{m} \leftarrow(1-\lambda) \frac{1}{N_{t}} \sum_{s=1}^{N_{t}} \gamma_{t s}+\lambda\left(\gamma_{t, \tau}+\frac{1}{(1-\alpha) N} \sum_{s=1}^{N_{t}} \zeta_{t}\left(\mathbf{r}_{t}(s)\right)\right) \\
& \left.\mathfrak{U}_{t k}^{i+1} \leftarrow\left\{\mathbf{x}_{t-1} \in \mathfrak{U}_{t k}^{i}:-\widetilde{g}_{t}^{m} \mathbf{x}_{t-1} \geq \widetilde{u}_{t k}\left(\overline{\mathbf{x}}_{t-1}^{m}\right)-\widetilde{\mathbf{g}}_{t}^{m} \overline{\mathbf{x}}_{t-1}^{m}\right)\right\}
\end{aligned}
$$

end for

\section{end for}

\section{[Upper bound update]}

$\bar{z} \leftarrow \max _{\mathbf{x}_{1}}\left\{\sum_{k=1}^{K} \mathfrak{U}_{2}^{i+1}\left(\mathbf{x}_{1} \mid k\right) P\left(k \mid \overline{\mathbf{r}}_{[T]}\right): \mathbb{1}^{\top} \mathbf{x}_{1}=\left(\mathbb{1}+\overline{\mathbf{r}}_{1}\right)^{\top} \mathbf{x}_{0}, \mathbf{x}_{1} \geq 0\right\}$

if $\mid\left(\bar{z} / \operatorname{lag}_{r}(z)-1 \mid<\epsilon\right.$ then

stb_count $\leftarrow$ stb_count +1

else

stb_count $\leftarrow 0$

end if

$i \leftarrow i+1$ 
The dynamic asset allocation process under uncertainty embeds a sequence of decision at each time succeeded by the asset return realization of the following period. In Figure 4.8, an illustrative decision tree depicts the described process. This example represent a generic time dependence of asset returns, since it is possible to consider different conditional probability distributions of $\mathbf{r}_{t+1}$ for each given $\mathbf{r}_{t}$.

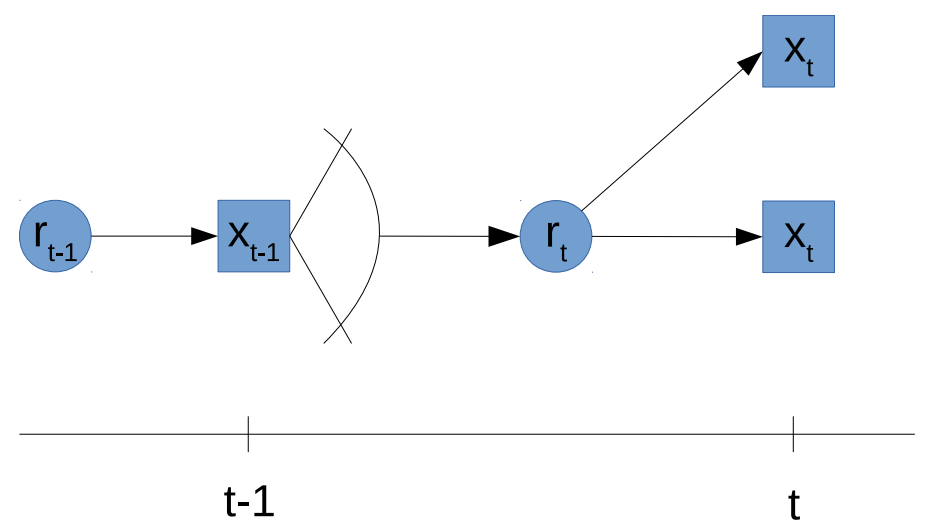

Figure 4.8: Decision tree of the generic problem with return dependence

In particular, when the return time dependence is modeled using HMM the decision process can be represented as in Figure 4.9.

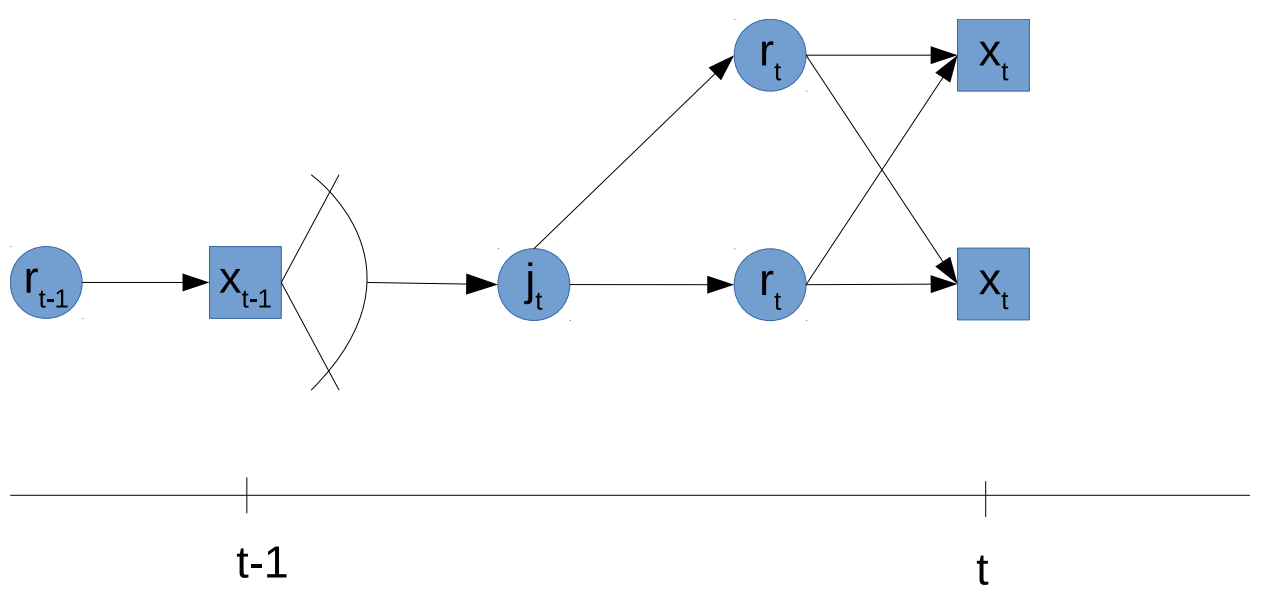

Figure 4.9: Decision tree of the problem with return dependence modeled with HMM

Note that, in Figure 4.9 the conditional allocation at a given time would depend on the whole history of asset returns, which would lead to an intractable optimization problem where the full tree must be represented. In Figure 4.10, represents an computationally tractable approximation of Figure 4.9, that can be solved by SDDP. 


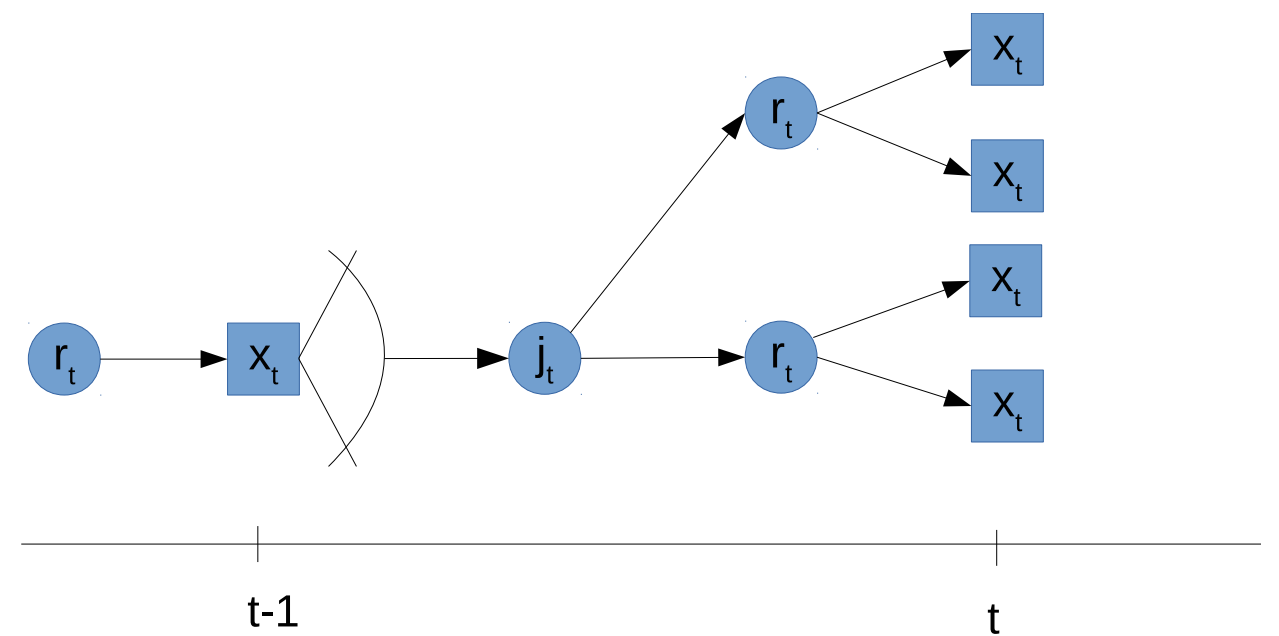

Figure 4.10: Decision tree of the problem of our proposal

\section{(d) Robust H2SDDP for asset allocation: Transaction costs, temporal dependence and ambiguity aversion}

As previously mentioned, it is well known that is difficult to reliably estimate the joint probability distribution of the returns, this uncertainty regarding the distribution can be perceive as ambiguity. Therefore, we propose a model that is robust to such uncertainty assuming that the investor would be averse to ambiguity.

For portfolio optimization, there are some approaches using ambiguity aversion over mean-variance model to mitigate the returns estimation errors $[85,86]$. Our methodology is an alternative to the previously mentioned methods in Chapter 2, to construct a robust model that reduces the sensitivity of Markowitz's optimal portfolio.

In the H2SDDP optimization model there is uncertainty about the likelihood of the states, thus our approach will be to estimate the interval of possible values for each state. It will be used those intervals on a robust optimization to obtain a portfolio less sensitive to changes in the returns distribution. This formulation is a generalization of the H2SDDP, presented in the previous Section 4.3(c).

The objective function is similar to (4.28), but in this case we will use a confidence interval for $P\left(k \mid \overline{\mathbf{r}}_{[1, t]}\right)$, that is $\underline{q_{k}} \leq P\left(k \mid \overline{\mathbf{r}}_{[1, t]}\right) \leq \overline{q_{k}}$. The robust model will choose the worst combination of $p_{k}$ between these values.

$$
\begin{gathered}
\min _{\mathbf{q} \in \mathcal{Q}} \sum_{k=1}^{K} U_{t}\left(\mathbf{x}_{t} \mid k\right) q_{k} \\
\mathcal{Q}=\left\{\mathbf{q} \in \mathbb{R}^{K} \mid \sum_{k \in K} q_{k}=1, \underline{\mathbf{q}} \leq \mathbf{q} \leq \overline{\mathbf{q}},\right\}
\end{gathered}
$$


Using (4.32) and (4.33) we can define our 2-stage robust problem as

$$
\begin{array}{rlr}
\max _{\mathbf{x}_{t} \in X\left(\mathbf{x}_{t-1}, \overline{\mathbf{r}}_{t}\right)} \min _{q} & \sum_{k=1}^{K} U_{t}\left(\mathbf{x}_{t} \mid k\right) q_{k} & \\
\text { s. t. } & \sum_{k} q_{k}=1 & : z \\
& -q_{k} \geq-\overline{q_{k}} & \forall k \in\{1, \ldots, K\}: y_{k} \\
& q_{k} \geq \underline{q_{k}} & \forall k \in\{1, \ldots, K\}: w_{k} \\
& x \geq 0 &
\end{array}
$$

The objective function has a nonlinearity due to the product of first and second stage variables $U_{t}\left(\mathbf{x}_{t} \mid k\right) q_{k}$. To solve the two-stage problem (4.34), first we have to formulate the dual problem of (4.32), using the dual variables $\left(z, w_{k}, y_{k}\right)$ we have

$$
\begin{array}{ll}
\max _{z, \mathbf{y}, \mathbf{w}} & z+\sum_{k=1}^{K}\left(\underline{q_{k}} w_{k}-\overline{q_{k}} y_{k}\right) \\
\text { s. t. } & w_{k}-y_{k}+z \leq U_{t}\left(\mathbf{x}_{t} \mid k\right) \quad \forall k \in\{1, \ldots, K\} \\
& \mathbf{w}, \mathbf{y} \geq 0, z \in \mathbb{R}
\end{array}
$$

Adding the restrictions from the original problem and transactional costs, we have

$$
\begin{aligned}
R_{t}^{P}\left(\mathbf{x}_{t-1}, \overline{\mathbf{r}}_{[t]}\right)=\overline{\mathbf{r}}_{t}^{\top} \mathbf{x}_{t-1} & \\
\max _{\mathbf{x}_{t}, \mathbf{y}, \mathbf{w}, \mathbf{d}_{t}^{+}, \mathbf{d}_{t}^{-}, z}-\sum_{i \in A} c\left(d_{t i}^{+}+d_{t i}^{-}\right)+z+\sum_{k=1}^{K}\left(\underline{q_{k}} w_{k}-\overline{q_{k}} y_{k}\right) & \\
\text { s. t. } \mathbb{1}^{\top} \mathbf{x}_{t}+\sum_{i \in A} c\left(d_{t i}^{+}+d_{t i}^{-}\right)=\left(\mathbb{1}+\overline{\mathbf{r}}_{t}\right)^{\top} \mathbf{x}_{t-1} & \\
& x_{t i}-d_{t i}^{+}+d_{t i}^{-}=\left(1+\bar{r}_{t i}\right) x_{t-1, i}, \\
& w_{k}-y_{k}+z \leq U_{t}\left(\mathbf{x}_{t} \mid k\right) \\
& \mathbf{d}_{t}^{+}, \mathbf{d}_{t}^{-}, \mathbf{x}_{\mathbf{t}}, \mathbf{w}, \mathbf{y} \geq 0, z \in \mathbb{R}
\end{aligned} \quad \forall i \in \mathcal{A} \backslash\{1\}
$$

This robust model is used only in forward procedure, since the state is unknown, it is necessary to estimate the likelihood of the states. In backward procedure the states are known, thus there is no uncertainty associated with it. 


\section{(e) H2SDDP for asset allocation: Transaction costs, tem- poral dependence and sell short}

Sell short consist in selling an asset that is not owned by the seller, betting on the fall in the asset price, to later buy the asset with a lower value, thus earning the difference between the sell price and the bought price. Sell short(selling and later purchasing) has the opposite properties of purchasing and later selling, in which the loss is limited and gain unlimited, in short selling the gain becomes limited but the loss is unlimited, since there is not an upper limit for the asset price.

However, in practice it know that asset price will not rise indefinitely, there is a reasonably limit for this value that can be evaluate, for example, using the CVaR. Allowing sell short in asset allocation optimization models enable the portfolio to allocate negative values for the assets, making possible to better exploit market opportunities. In many markets the sell short involves renting the asset until the asset is purchase and returned for who bought.

Sell short is very important for quantitative models, as with it is possible to succeed even when the market has downward trend. For example, short sell allows the model to obtain a positive expect value for the portfolio even in situations when all assets have negative expected returns. Actually, using short sell the model can take advantage of these situations, and without it the only reasonable alternative is to invest on risk-free asset, a downward trend is useful as upward trend when using sell short. It also allows a some leverage behavior, by short selling an asset in order to obtain cash to invest in other assets. With $x_{t i}^{-}$being the negative allocation of asset $i$, the short sell, and $x_{t i}^{+}$the positive, also we consider the rent of sell short with penalization in objective function costing $c_{s}$

$$
\begin{aligned}
& u_{t k}\left(\mathbf{x}_{t}, \mathbf{r}_{t+1}(s)\right)=\overline{\mathbf{r}}_{t}^{\top} \mathbf{x}_{t-1}+ \\
& \max _{\mathbf{x}_{t}^{+}, \mathbf{x}_{t}^{-}, \mathbf{d}_{t}^{+}, \mathbf{d}_{t}^{-}}-c_{s} \sum_{i \in A} x_{t i}^{-}-\sum_{i \in A} c\left(d_{t i}^{+}+d_{t i}^{-}\right)+\sum_{j=1}^{K} \mathfrak{U}_{t+2}\left(\mathbf{x}_{t+1} \mid j\right) P(j \mid k) \\
& \text { s. t. } \mathbb{1}^{\top} \mathbf{x}_{t}+\sum_{i \in A} c\left(d_{t i}^{+}+d_{t i}^{-}\right)=\left(\mathbb{1}+\overline{\mathbf{r}}_{t}\right)^{\top} \mathbf{x}_{t-1} \\
& \sum_{i \in A} x_{t i}^{-} \leq\left(\mathbb{1}+\overline{\mathbf{r}}_{t}\right)^{\top} \mathbf{x}_{t-1} \\
& x_{t i}^{+}-x_{t i}^{-}=x_{t i} \quad \forall i \in A \\
& x_{t i}-d_{t i}^{+}+d_{t i}^{-}=\left(1+\bar{r}_{t i}\right) x_{t-1, i}, \quad \forall i \in \mathcal{A} \backslash\{1\} \\
& \mathbf{d}_{t}^{+}, \mathbf{d}_{t}^{-}, \mathbf{x}_{t}^{+}, \mathbf{x}_{t}^{-} \geq 0
\end{aligned}
$$

Analogous, using the constrains (4.38), (4.39) and the rent on the 
objective this can be formulated for the forward step (4.30). Notice that the constrain (4.38) makes this a problem without complete recourse, because the right side can be negative. But it occurs in a very rare occasion when the whole portfolio in negative.

\subsection{Computational Experiments}

To analyze how the proposed methods would behave in practice, we will test and simulate with real data. The data sets used in the experiments consist of industrial portfolios, the stocks are grouped according to the industry in which belongs, with stocks from NYSE, AMEX and NASDAQ ${ }^{3}$. The stock weight in the portfolio is proportional to its market value. We will use monthly data of 5 industrial portfolios(Cnsmr, Manuf, HiTec, Hlth and Other) and daily data for 10 industrial portfolios (NoDur, Durbl, Manuf, Enrgy, HiTec, Telcm, Shops, Hlth, Utils and Other), and also the risk free asset with $0 \%$ of return.

Additionally, we used HMM and the k-means ${ }^{4}$ of the machine learning library Mlpack [87] and the Latin Hypercube Sampling method from Matlab. The multivariate Gaussian mixture of the HMM was estimated considering the log-normal distributions of the historical returns, but in the optimization problem was transformed to be accordingly to the real return distribution.

Algorithms were implemented in $\mathrm{C}++$ language, using $\mathrm{CPLEX}{ }^{5}$ to solve the linear problems and also some auxiliary functions of the Armadillo library [88]. All the experiments were conducted on Intel quad-core i5-3570 3.4 GHz with 16GB RAM machine, only one core were used during the optimization.

The experiments are organized as follows: first it will be presented some information about the data, used in this work, some metrics and the historical returns of the financial time series. The following section will compare two sampling methods, the Monte Carlo and the Latin Hypercube Sampling. Later in this section, the model's sensibility to variations on the parameters will be tested, further investigating on how the results and optimal solution behaves. Finally, out of sampling simulation is done to compare the presented methods.

\section{(a) Data analysis}

Two data sets was used on our experiments, a monthly and another daily, both from Kenneth R. French data set ${ }^{6}$. First tests we will use monthly data from January 1970 to December 2014 for 5 industrial portfolios. In Table 4.1

\footnotetext{
${ }^{3}$ http://mba.tuck.dartmouth.edu/pages/faculty/ken.french/data_library.html

${ }^{4}$ Used to initialize the emissions distributions of the HMM

${ }^{5}$ http://www-01.ibm.com/software/integration/optimization/cplex-optimizer/

${ }^{6} \mathrm{http}: / /$ mba.tuck.dartmouth.edu/pages/faculty/ken.french/data_library.html
} 
we exhibit some metrics about the return series of the data set industrial portfolios. The second data set consists in daily returns for 10 industrial portfolio, and Table 4.2 contains a summary of the data.

\begin{tabular}{l|r|r|r|r|r}
\hline Measure & Cnsmr & Manuf & HiTec & Hlth & Other \\
\hline Max & 21.73 & 17.28 & 19.98 & 29.52 & 20.22 \\
Min & -25.03 & -20.80 & -22.65 & -20.46 & -23.60 \\
Mean & 1.04 & 1.00 & 0.93 & 1.06 & 0.94 \\
StdDev & 4.66 & 4.44 & 5.71 & 4.96 & 5.39 \\
VaR & 4.22 & 4.22 & 5.67 & 4.90 & 5.45 \\
CvaR & 7.64 & 7.45 & 10.04 & 8.02 & 9.28 \\
\hline
\end{tabular}

Table 4.1: Monthly data series for 5 industrial portfolios

\begin{tabular}{|c|c|c|c|c|c|c|c|c|c|c|}
\hline Measure & NoDur & Durbl & Manuf & Enrgy & HiTec & Telcm & Shops & Hlth & Utils & Other \\
\hline Max & 10.26 & 9.73 & 10.75 & 19.33 & 16.06 & 14.51 & 10.99 & 11.09 & 14.43 & 11.27 \\
\hline Min & -17.03 & -18.35 & -20.00 & -19.43 & -19.98 & -16.68 & -16.74 & -17.89 & -12.86 & -15.24 \\
\hline Mean & 0.05 & 0.04 & 0.05 & 0.05 & 0.04 & 0.05 & 0.05 & 0.05 & 0.04 & 0.04 \\
\hline StdDev & 0.91 & 1.37 & 1.09 & 1.36 & 1.48 & 1.14 & 1.10 & 1.12 & 0.88 & 1.19 \\
\hline VaR & 0.93 & 1.42 & 1.11 & 1.38 & 1.58 & 1.13 & 1.16 & 1.19 & 0.80 & 1.15 \\
\hline CVaR & 1.58 & 2.40 & 1.91 & 2.34 & 2.62 & 1.97 & 1.93 & 1.97 & 1.52 & 2.08 \\
\hline
\end{tabular}

Table 4.2: Daily data series for 10 industrial portfolios

To make it easier to visualize and compare the measures, we presented data as bar charts on Figure 4.11 and Figure 4.12.

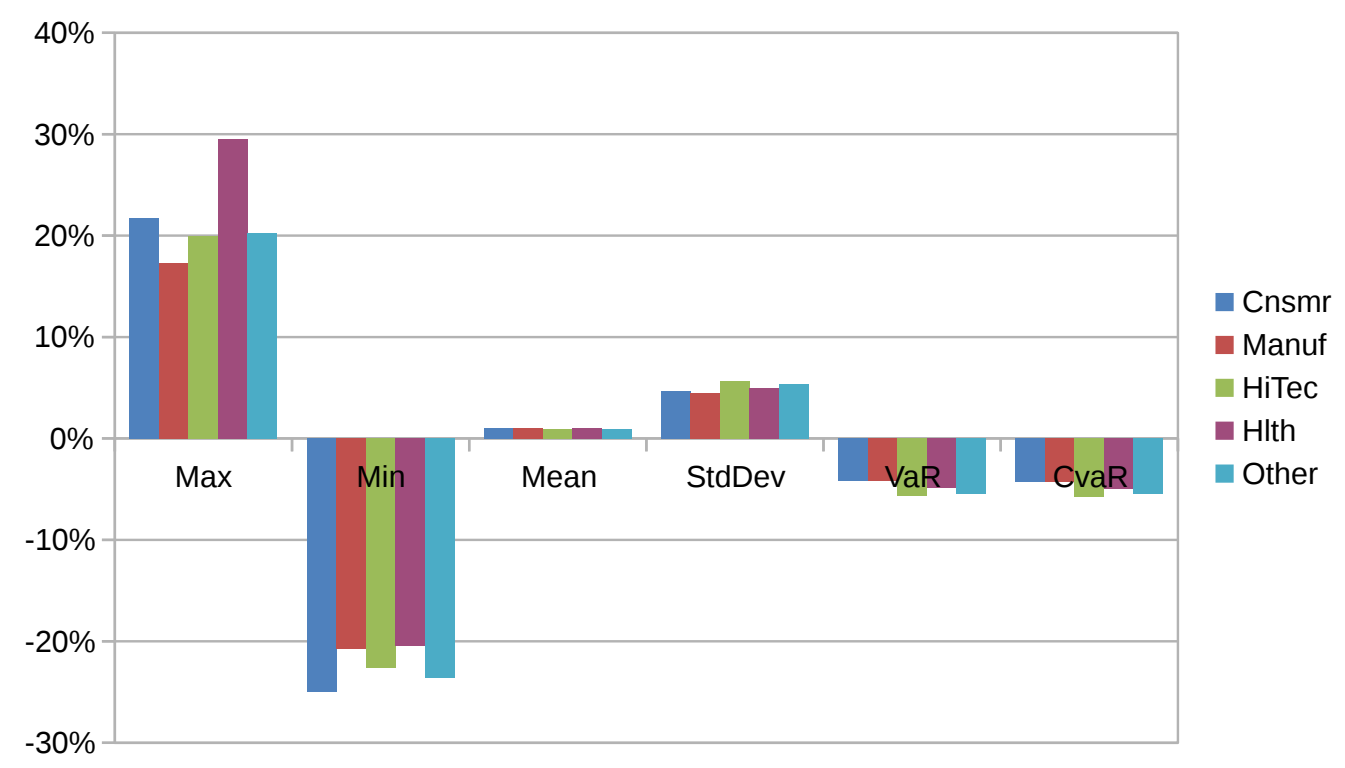

Figure 4.11: Metrics for historical monthly return series of the five industrial portfolios 


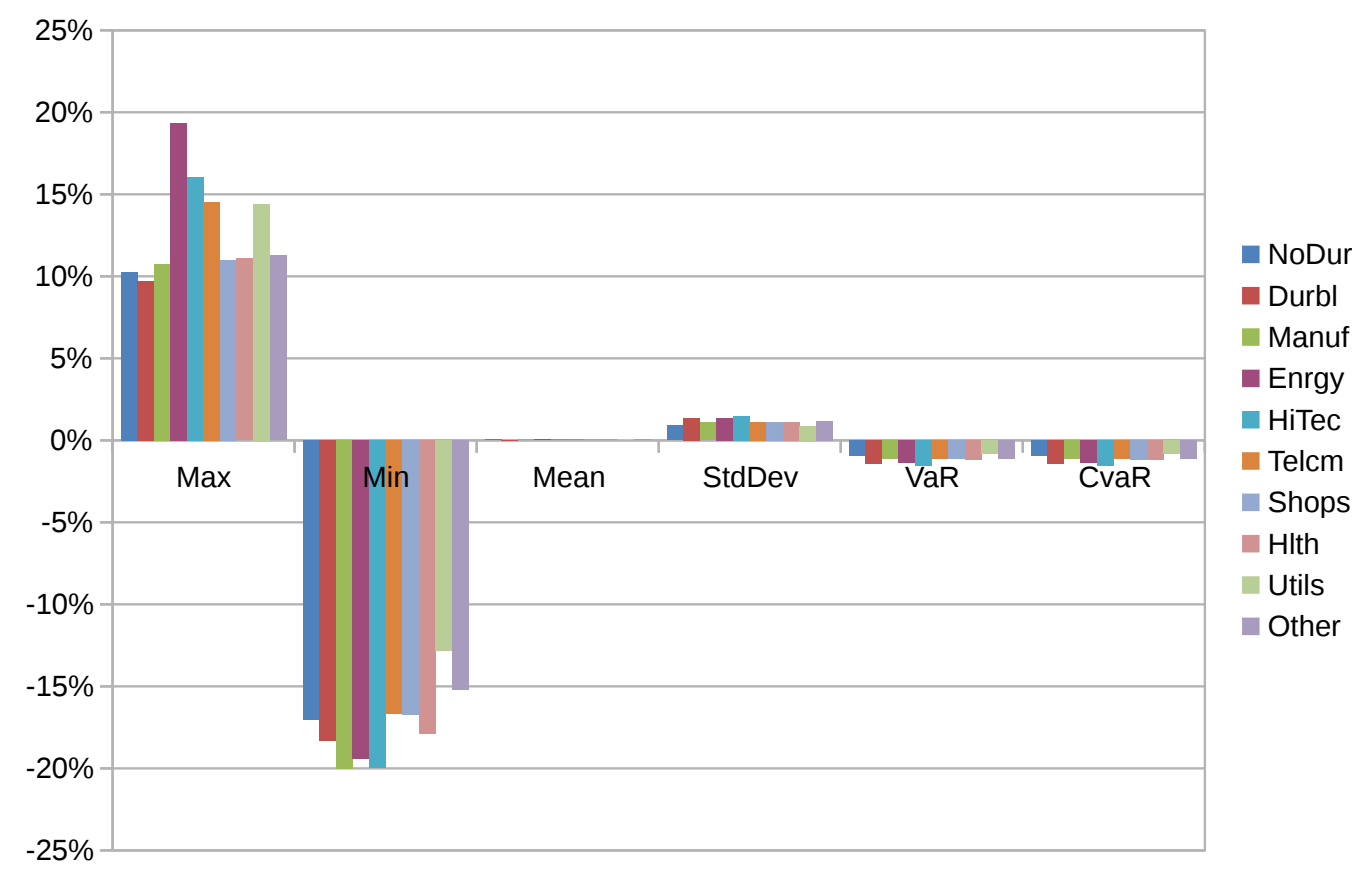

Figure 4.12: Metrics for historical daily return series of the ten industrial portfolios

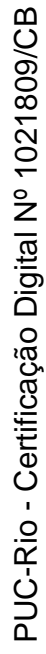

It is also important to present the financial time series of these data sets, where Figure 4.13 and Figure 4.14 show the monthly and daily cumulative performance from 1970 to 2014 for 5 and 10 industrial portfolio, respectively.

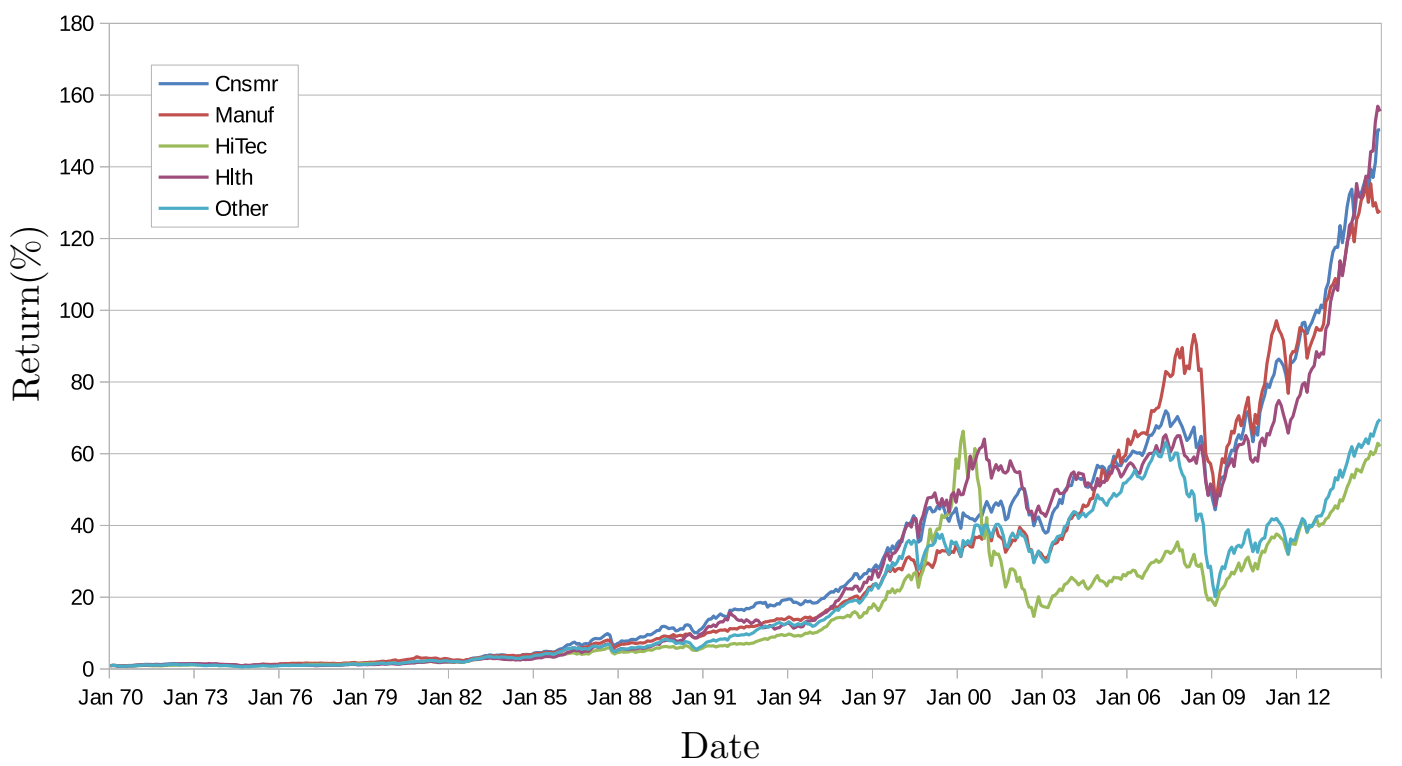

Figure 4.13: Cumulative performance for monthly data set of the five industrial portfolios 


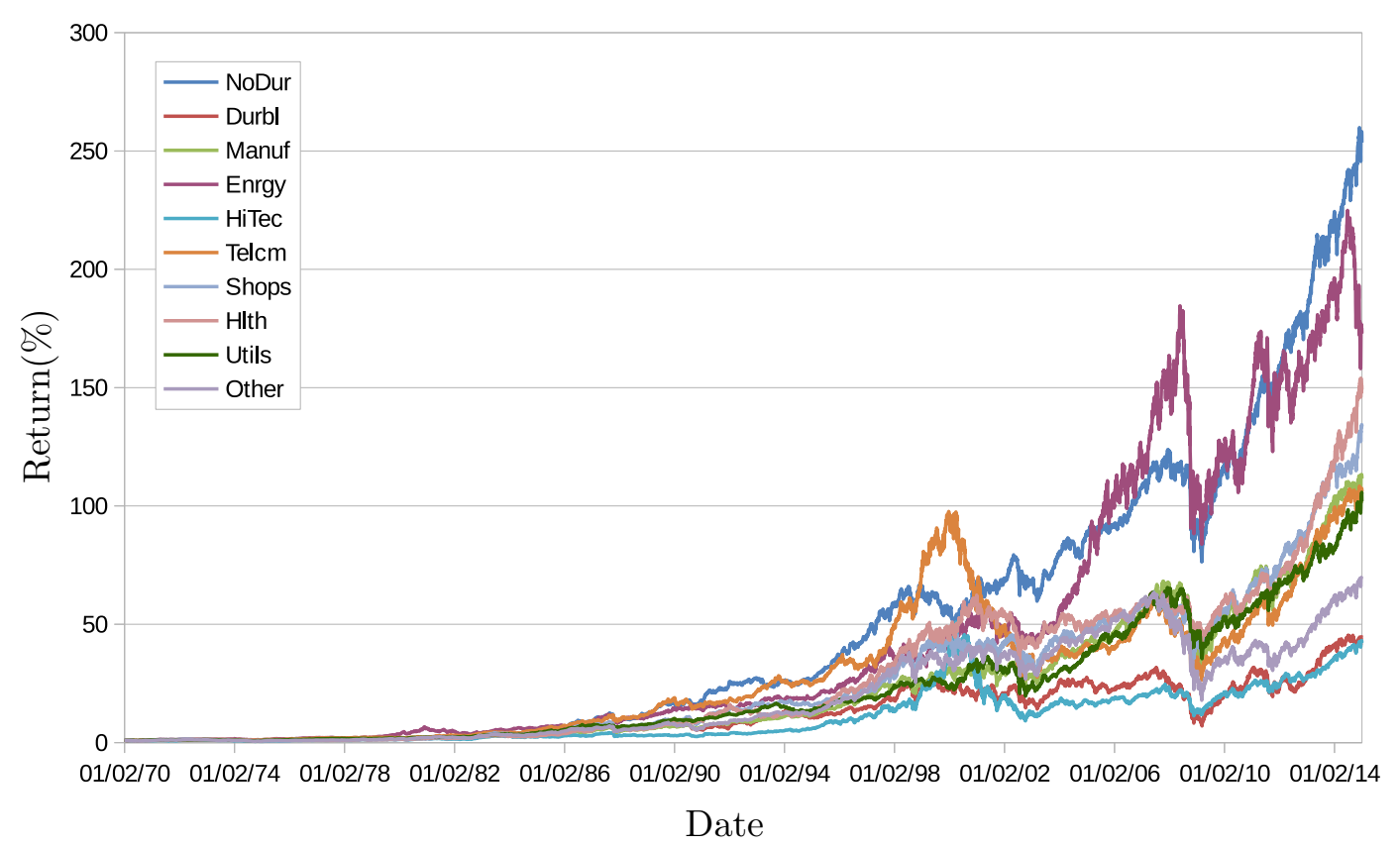

Figure 4.14: Cumulative performance for daily data set of the ten industrial portfolios

\section{(b) Sampling Methods}

As discussed before, Latin Hypercube Sampling is a promising technique to substitute the Monte Carlo sampling in SAA techniques. Latin Hypercube Sampling is especially important for this optimization problem, because it is crucial to represent the distributions with least samples as possible, otherwise it will lead to a heavy computational burden.

To compare the results of the different sampling methods, we prepared an experiment that consists in running the optimization multiple times, comparing the average and standard deviations of the final upper bound. It was used the monthly data set (from 1970 to 2011), with six stages, three states, $\lambda 0.1, \alpha 0.9$ and no transaction cost. The test was done for Monte Carlo and Latin Hypercube Sampling, it gradually increase number of the samples per state $\{20,100,1000,2500,3000,3500,4000,4500,5000,7000\}$ repeating 5 times, for each one of this values, but with different random seeds. Notice, that the final total number of samples depends on the number of states, for example, with 2500 samples per state and three states the problem will have a total of 7500 samples.

We did an extensive amount of tests using Monte Carlo. However, even with 5,000 samples per stage, the standard deviation was still high. In Figure 4.15 we compare, using the average of the upper bound for the wealth 
and the standard deviation of it, the two sampling selection techniques for different numbers of samples.
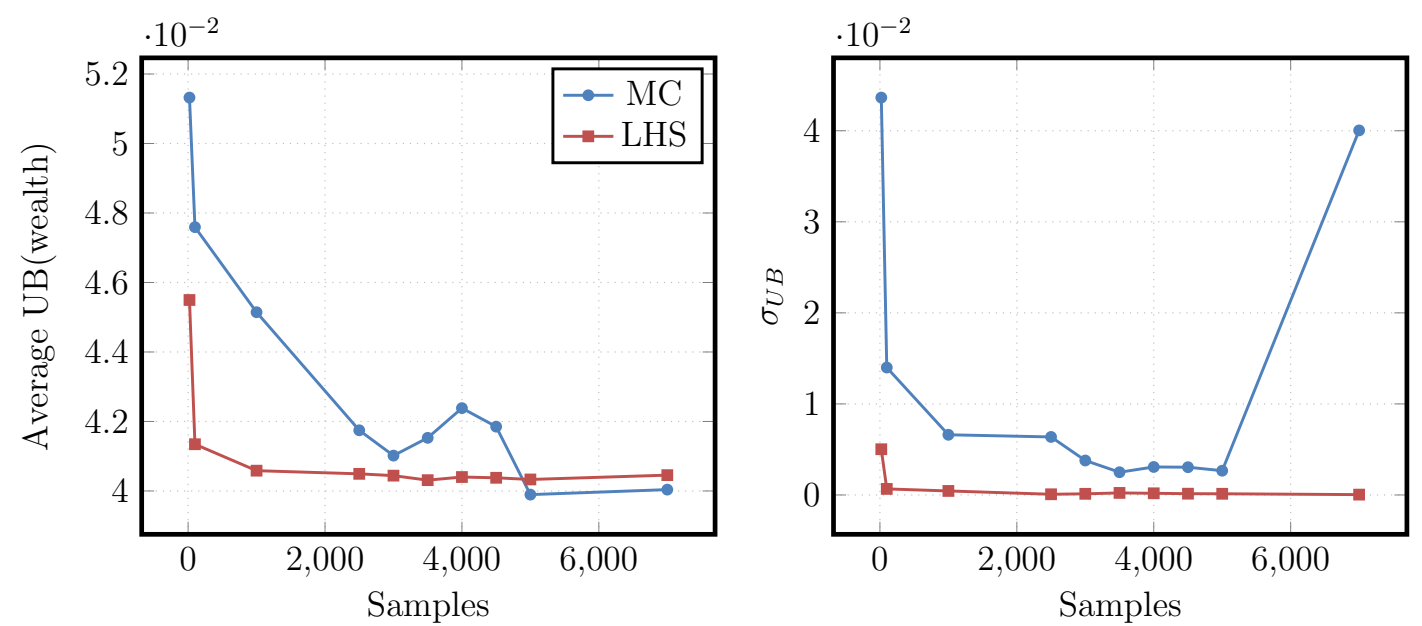

Figure 4.15: Comparison between Latin Hypercube Sampling (LHS) and Monte Carlo (MC)

It seems that with more samples, Monte Carlo gets a better average. However, running with 10,000 samples it reaches 0.0407858 , which is much closer to the Latin Hypercube Sampling value with 5,000 samples, and even with 10,000 samples the standard deviation still was very high.

As the solution of the problem is very stable using Latin Hypercube Sampling, to get the same stability with Mote Carlo it would require much more samples, this would increase the convergence time of the SDDP. Representing the distribution with last realizations, as possible, can substantially reduce computational times. Thus, we find the Latin Hypercube Sampling more adequate sampling method to be used for this model and choose to use 2500 samples per state as it seems that using more samples does not improve significantly the upper bound.

\section{(c) Sensitivity Analysis}

In addition to choosing the sampling method, there are many parameters that need to be selected to be able to implement the proposed methods. We need to select SDDP parameters such as number of stages, samples number, number of trials, risk aversion coefficient and many others. It is also necessary to analyze the model sensibility to variations, and if the model will behave as expected on this tests.

The number of samples $N_{t}$ can be different for each stage, but for the sake of simplicity, the same number of sample has been considered for all stages in this work, i.e $\left|N_{t}\right|$ is the same for $t \in \mathcal{H}$. Also, for all tests it was used the monthly data set (from 1970 to 2011), with the default parameters as six 
stages, three states, 2500 samples per state, $\lambda 0.1, \alpha 0.9$ and no transaction cost.

\section{HMM}

Moreover, there are other parameters from HMM that have to have some initial good guessing of the states emission probabilities. We employ k-means method to pick some centroids for every state and adopt these distributions as the initial guess for HMM. To determine the number of states, we did a sliding window validation for every number of states between 1 and 8 , selecting the best log-likelihood.

Figure 4.16 shows the likelihood of each state for the monthly historical train data set.

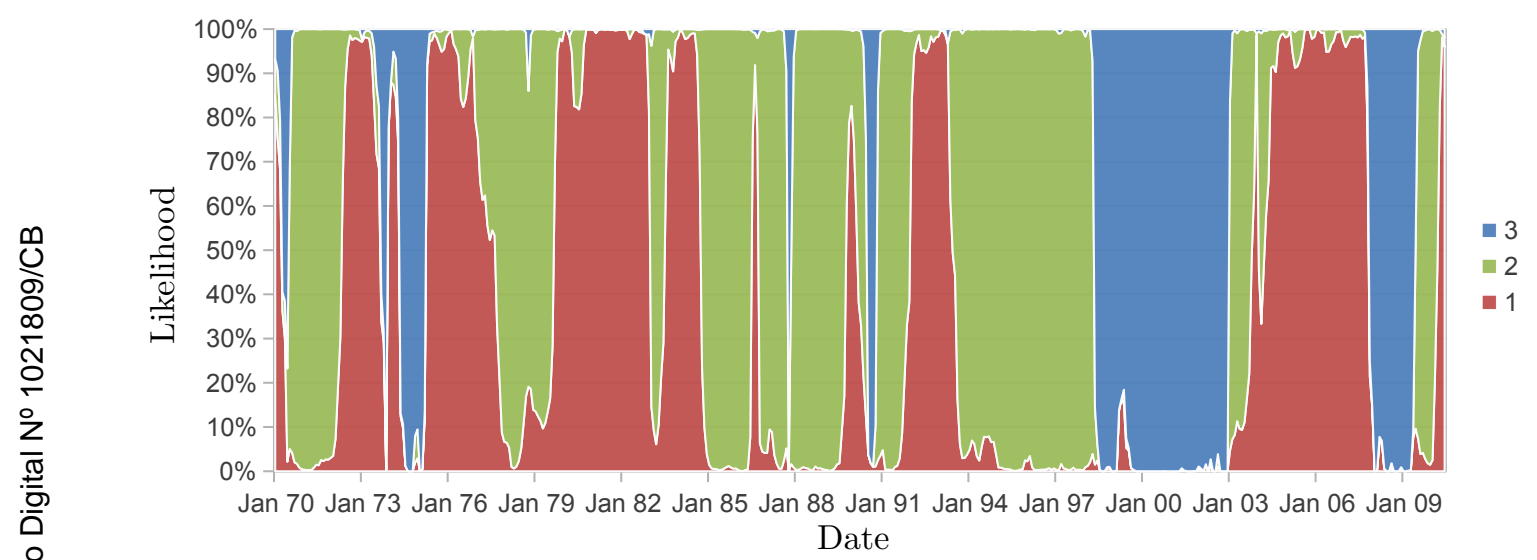

Figure 4.16: States likelihood for train data

We can see that the state transitions are not smooth, it seems to be because we are using k-means as our initial emissions distributions for the HMM, since k-means tries to minimize the number of intersections in the clusters. We should expect more diversified results if the samples were classify instead of using clusters.

\section{Impact of the Risk Aversion}

The risk aversion coefficient $(\lambda)$ also has big impact in the upper bound, that is the certainty equivalent of the portfolio, which can be interpreted as the fund worth. In Figure 4.17 we present the upper bound for different $\lambda$ values, for monthly returns on left and on right for daily returns. This results shows how the value of the portfolio changes for investors with different aversion to risk. 

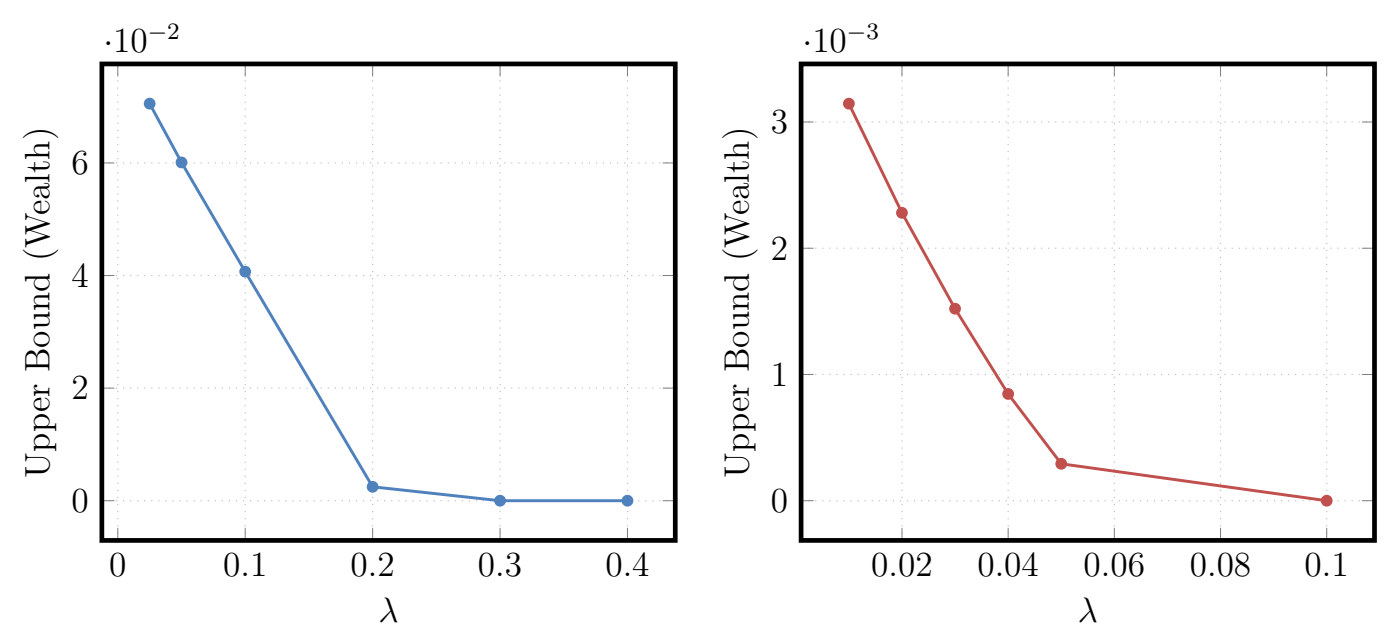

Figure 4.17: Impact of the risk aversion coefficient $(\lambda)$ on the upper bound for monthly returns and daily returns, respectively on the left and right

As expected, to have the same return as in monthly data set, the risk aversion coefficient has to be lower for the daily returns. For daily returns when $\lambda=0.1$ the optimal solution is to allocate everything on the risk-free asset, the correspondent value for monthly returns is 0.3 . The risk aversion coefficient should get higher when allowing short sell.

\section{Convergence and trials}

Since the number of possible scenarios increases exponentially with the number of stages, it is important to investigate how the number of stages affects the computational time, convergence and also the final solution. As the number of stages increase the computational time should also rise, since H2SDDP needs more iterations to converge. In Table 4.3 we present upper bound, time and number of cuts according to number of states, time is presented in minutes. The computational time needed to converge the SDDP get really high. Observe

\begin{tabular}{c|c|c|c}
\hline n. stages & upper bound & time & n. cuts \\
\hline 12 & 0.0772493 & 41 & 50 \\
24 & 0.15515 & 149 & 75 \\
48 & 0.324179 & 356 & 82 \\
96 & 0.754923 & 1088 & 109 \\
\hline
\end{tabular}

Table 4.3: Number of stages and the computational time

that with twice more stages the number of cuts increases slightly and the computational time increases substantially.

We did some prior tests to check whether it would be better to use many trials or only one trial at a time. This also determine the number of cuts added at each stage. Three ways of choosing the number of trials were tested; first 
using only one trial, second using 5 trials and, finally, using 5 trials as the upper bound becomes stable for 10 iterations. From our limit experiments, using more than one trial, i.e., adding multiple cuts, increase the convergence time even with the alternative approach using dynamic number of trials. It seems better to do $C$ iterations adding one cut per iteration than to add $C$ cuts on one iteration, for the convergence of the algorithm. The real advantage of adding multiple cuts could be on the quality of the worth function approximation, this could enhance stability of primal solutions.

The used method to test the convergence of SDDP was the stabilization of the upper bound. Whether, the upper bound of 10 iterations ago $z_{i-10}$ is stable for 5 iterations, i.e., 15 iterations without change greater than $\epsilon$. Notice that the upper bound of the iteration $i-10$ is compared with the current $i$ upper bound value. We opted for a tighter convergence criteria with $\epsilon=0.0001$ than do more tries. To illustrate the convergence of the H2SDDP, Figure 4.18 exhibits the upper bound changes, in percentage, for optimization of the H2SDDP with 96 periods.

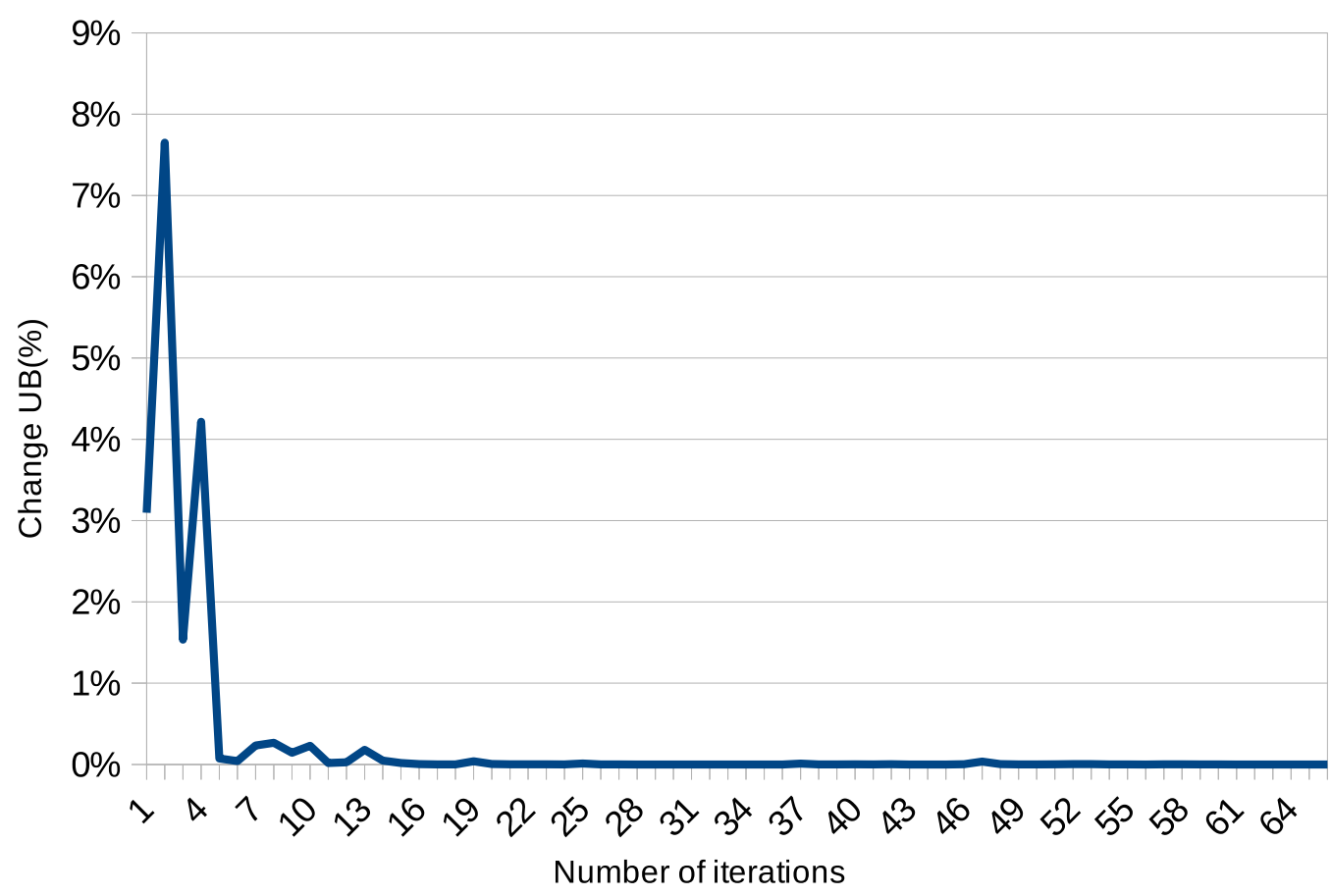

Figure 4.18: Convergence of the Upper Bound for monthly data set

\section{Transactional costs}

Another interesting subject is the impact of the transactional costs. First, we present how of the upper bound is sensible to variations of the transactional costs on Figure 4.19. Later we will discuss further implications of taking the transactional costs into account. It was used the monthly data set (from 1970 
to 2011), with six stages, three states, 2500 samples per state, $\lambda 0.1, \alpha 0.9$ and no transaction cost.

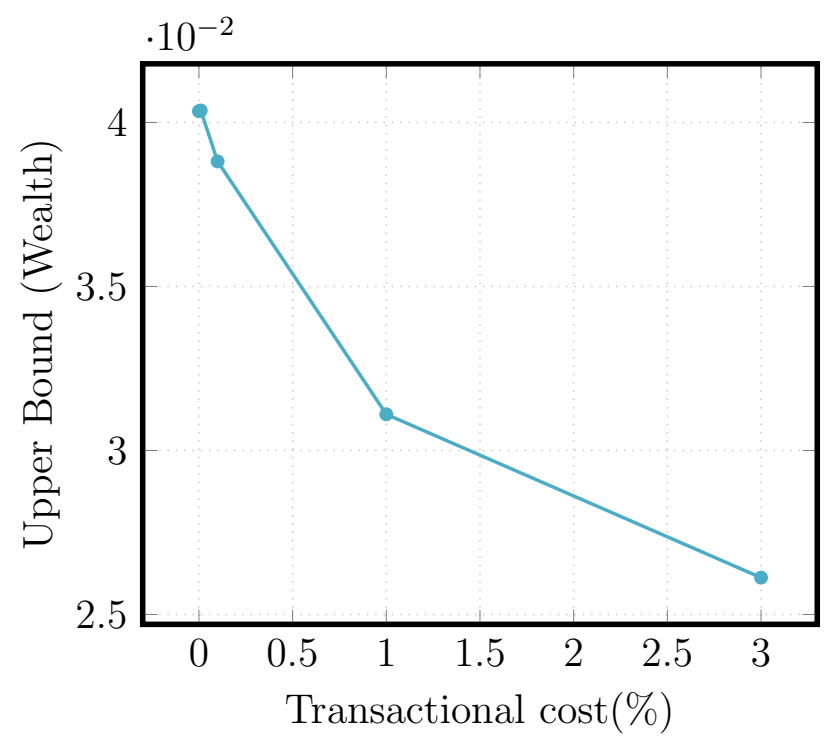

Figure 4.19: Impact of the transactional costs on the upper bound

It is evidently that the transactional costs can really impact the optimal solution. Since the return is uncertain but the transactional cost is not, higher transactional cost makes not worth to do some small changes on the investments, thus the model only invest when the expect return is higher enough to mitigate the costs involved.

Further analyzing how the transactional costs will affect the optimal solution. The portfolio allocation can be seen on Figure 4.20 with no transaction costs, and in Figure 4.21 with transactional cost equal to $0.1 \%$. Even at a cost of $0.1 \%$, smaller deviations are avoided when the model does not expect a significant gain with changing the portfolio. In Figure 4.20 and Figure 4.21 we can clearly see the portfolio stabilization effect caused by transaction costs, it is evident the resemblance to a regularization L1. 


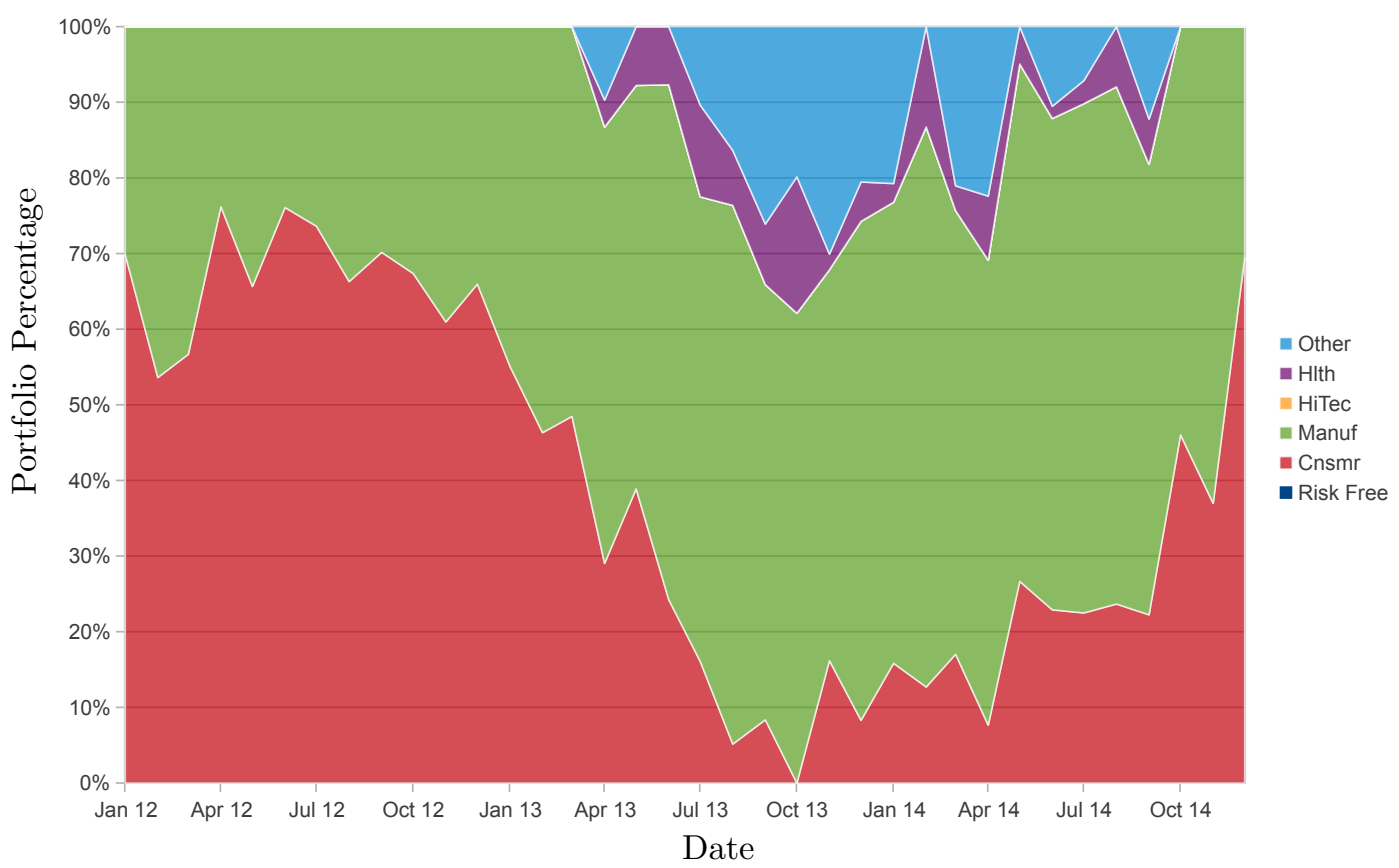

Figure 4.20: Portfolio allocation without transactional costs

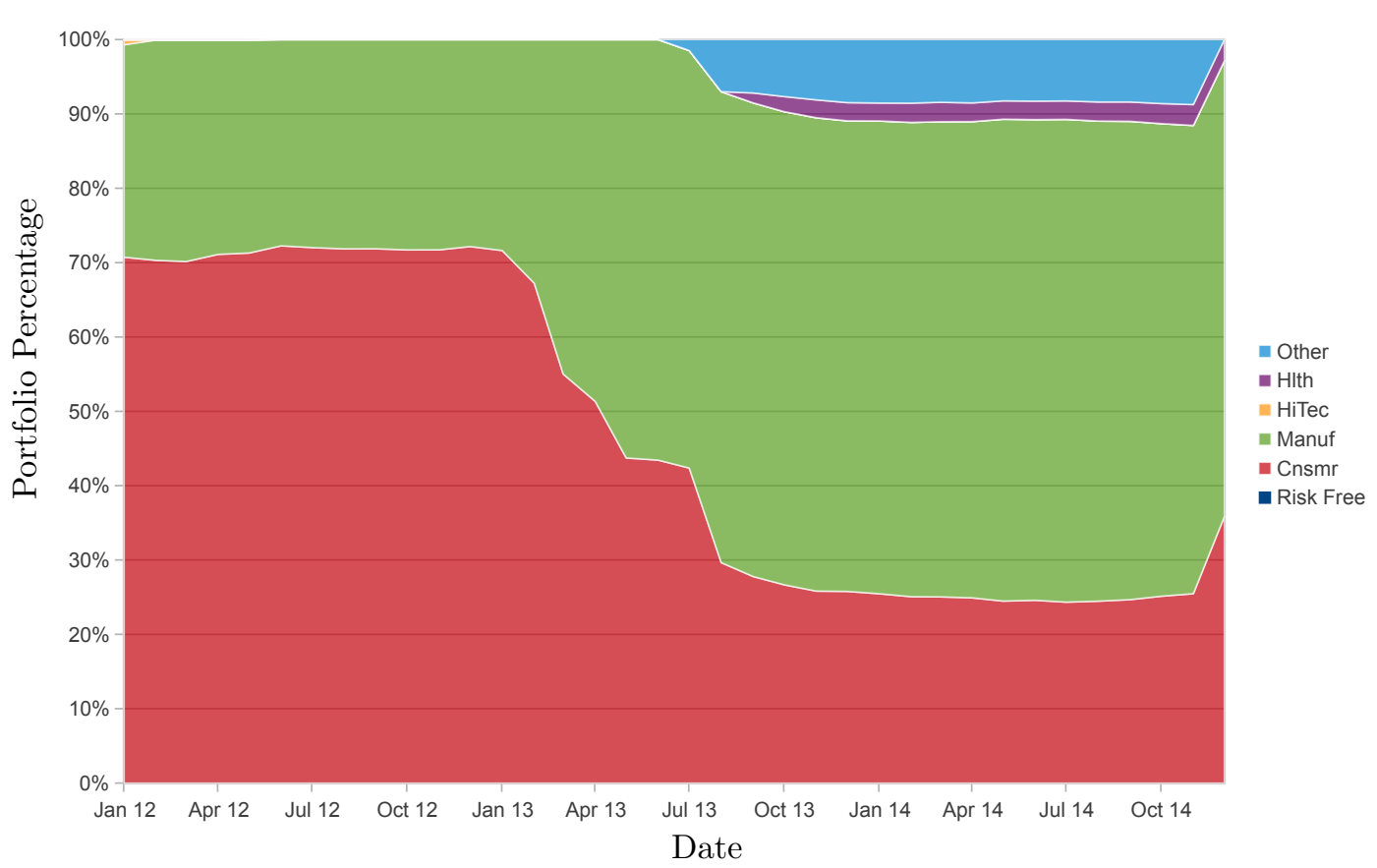

Figure 4.21: Portfolio allocation with transactional costs

\section{(d) Models Evaluation}

This section present the final results of simulations on out of sample data, in order to do evaluate the performance of the models. We will compare H2SDDP with different approaches and present the results for ambiguity aversion model. The parameters for the experiments on this sections are: 2500 
samples per stage, three states form the HMM, lambda 0.1, alpha 0.9 and transactional cost $0.1 \%$, it will be mentioned if there is any changes on this parameters. As the initial wealth is one, this value can also be interpreted as the return of the policy in percentage.

\section{Experiment with Monthly Data Set 2012 to 2014}

On the first tests we are going to use the monthly data set, training with dates from 1970 to 2011 and simulating with 2012 to 2014 (36 months). In H2SDDP simulations the train data set(1970 to 2011) was used to evaluate the policy, then the policy was used to evaluate the performance on test data $\operatorname{set}(2012$ to 2014$)$.

To compare the performance of the policy accordingly to the risk aversion coefficient, we alternate the $\lambda$ value to evaluate what would be the behavior in out of samples data, the result is presented in Table 4.4.

\begin{tabular}{c|c|l}
$\boldsymbol{\lambda}$ & CVaR & Ret. \\
\hline 0.2 & 4.16 & 155.72 \\
0.1 & 4.14 & 158.34 \\
0.09 & 4.18 & 160.79 \\
0.08 & 4.04 & 162.29 \\
0.07 & 4.06 & 166.35 \\
0.06 & 4.12 & 165.04 \\
0.05 & 4.31 & 170.45 \\
0.025 & 4.75 & 171.36
\end{tabular}

Table 4.4: CVaR and return values of the simulated polices for different risk coefficients, results in percentage

In order to analyze and compare the proposed approaches, besides of simulating the previous presented models we also evaluate the results for the equally weight portfolio and the myopic strategy. One can easily underestimate the value of the equally weighted portfolio but in practical situations they outperform most of the traditional optimization approaches [89]. Important to mention, since we are considering equal distribution of returns for each stage, the myopic method has the same portfolio in percentage for the entire period, then the myopic portfolio was establish with a 2-stage optimization.

Figure 4.22 show the result of the simulations. This is an experiment using the monthly data set comparing H2SDDP with $\lambda 0.1$ and 0.05 with the myopic and equally weight policies. 


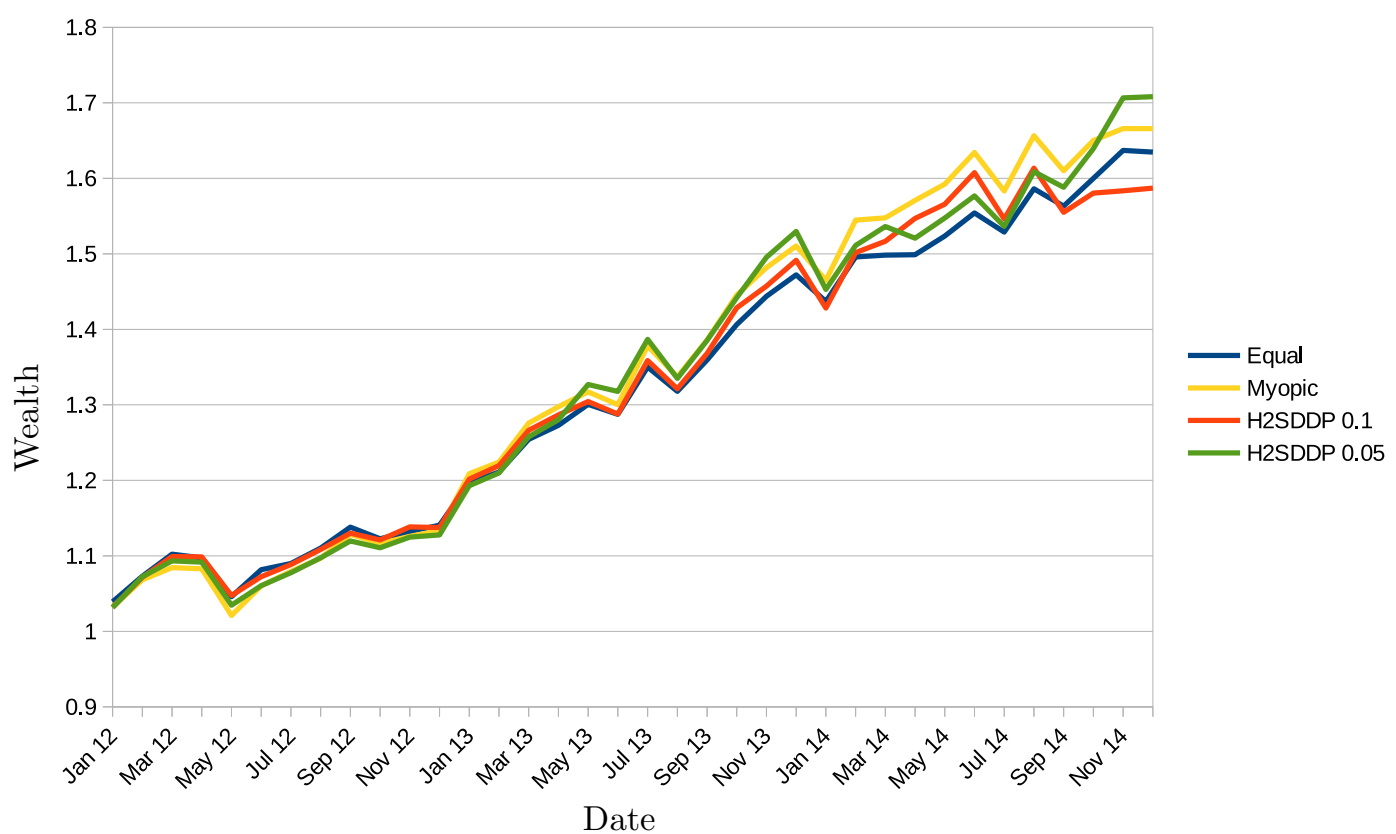

Figure 4.22: Comparing the different methods for asset allocation data form 2012 to 2014

Further investigation is required to interpret the results of Figure 4.22. Inspecting the assets cumulative returns on Figure 4.23 for the same time period we can observe that this was an interval of strong upward trend and, furthermore, all assets has also very similar behavior for this period. This hypothesis also is sustained by results presented on Table 4.4, where the greatest return is obtained with $\lambda=0.025$. In this scenario, it is expected to have similar results for all the presented methods.

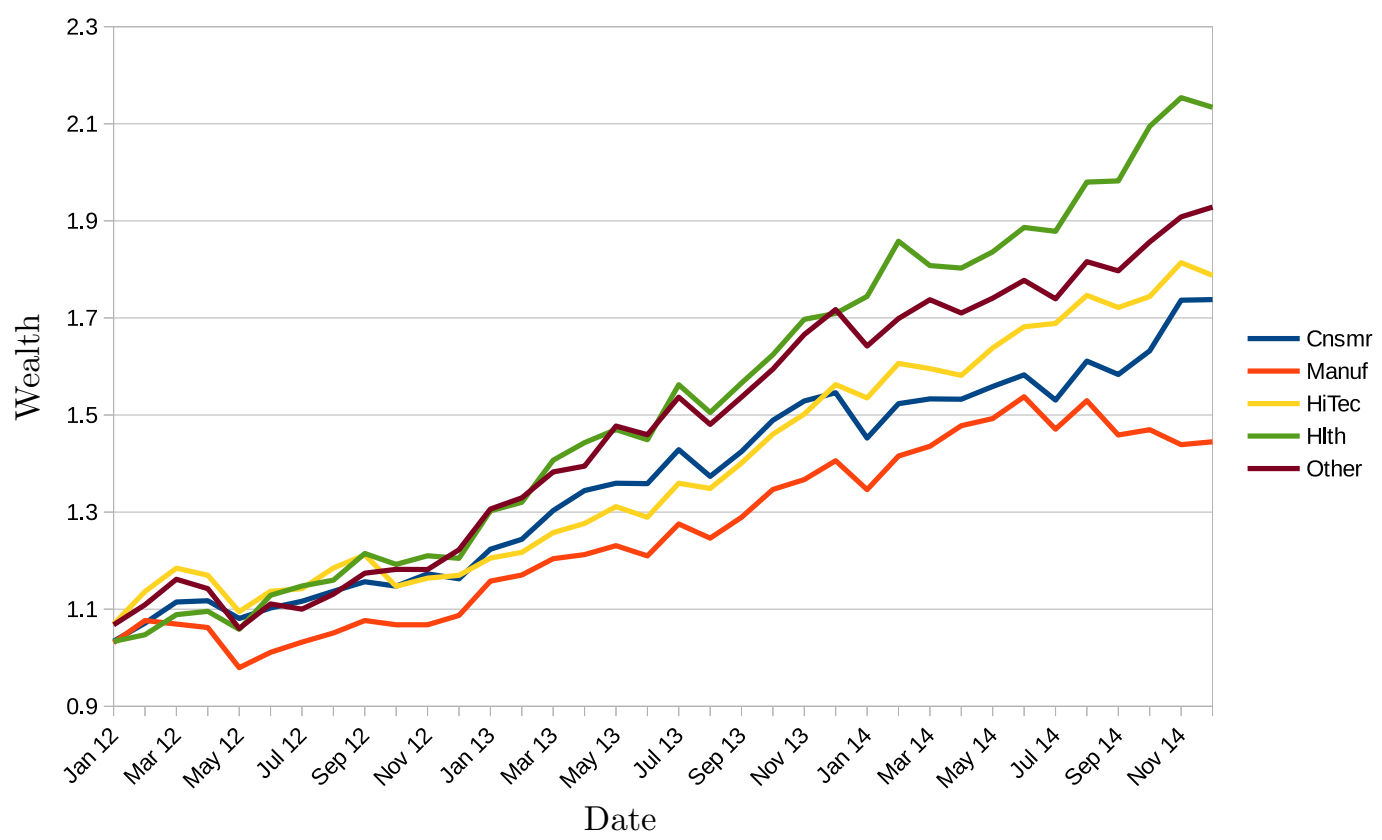

Figure 4.23: Cumulative returns series from January 2012 to December 2014 


\section{Experiment with Monthly Data 2007 to 2014}

To test this interpretation we develop a new test case now simulating on data from 2007 to 2014, as in this period there is more volatility, and changes in trends, the dynamic policy should be capable of changing the portfolio to adapt to this variations. The models uses samples from 1970 to 2006 and 96 stages, after finding the optimal solution the approximations of the expected worth functions are used on the test with 96 months, January 2007 to December 2014. The cumulative performance for the industrial portfolio from this period is exhibit in Figure 4.24. The transactional costs was account to all the models, for those that do not consider in the optimization model it was discounted the costs after the optimization.

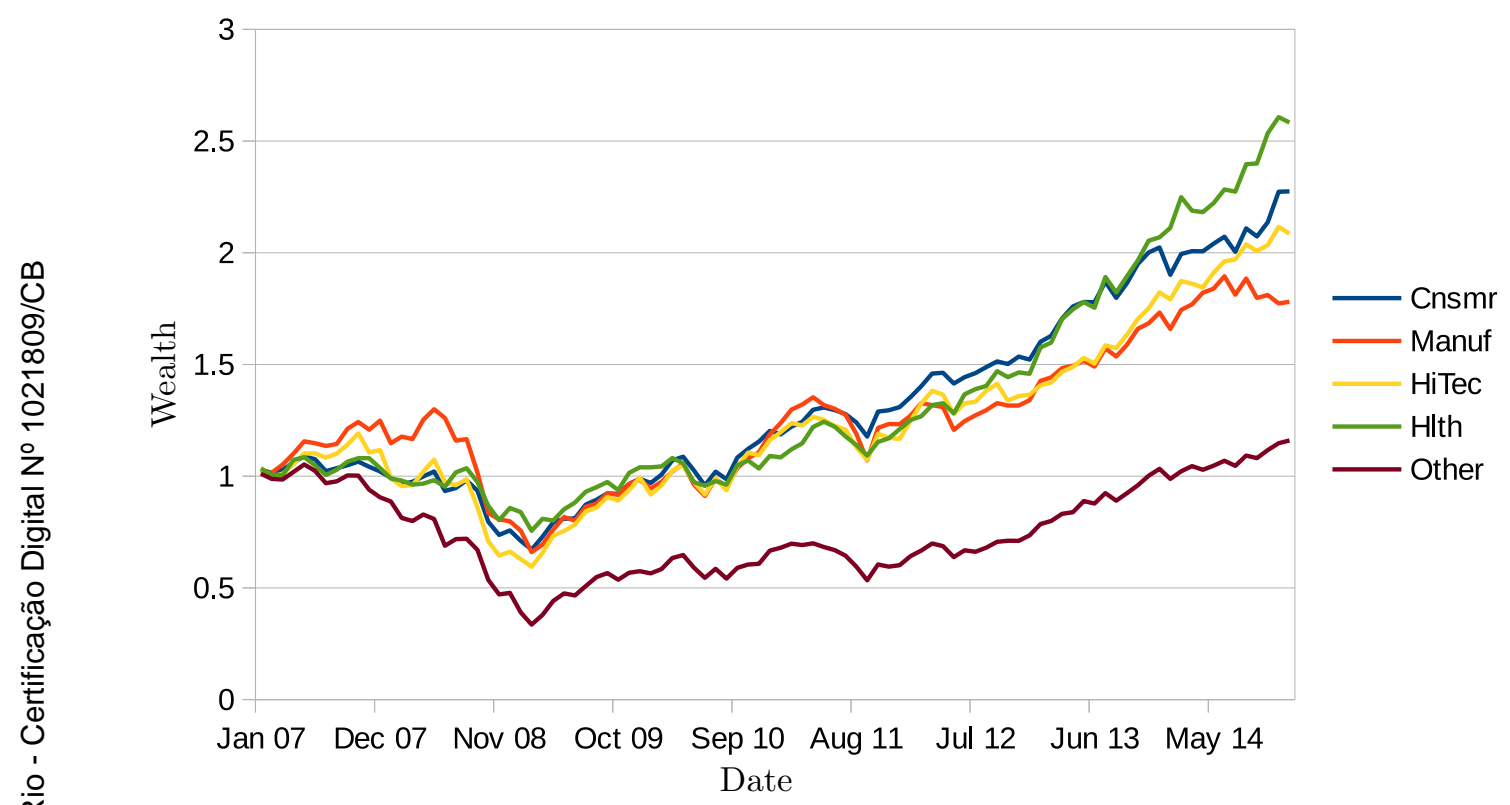

Figure 4.24: Returns series from January 2007 to December 2014

In this experiment, on Figure 4.25 beside the H2SDDP, Equally weighted and Myopic models, it is also presented the performance of the Robust model, using and interval of $10 \%$, i.e. $0.9 \times P\left(k \mid \overline{\mathbf{r}}_{[1, t]}\right) \leq q_{k} \leq 1.1 \times P\left(k \mid \overline{\mathbf{r}}_{[1, t]}\right), \forall k \in$ $\{1, \ldots, K\}$. 


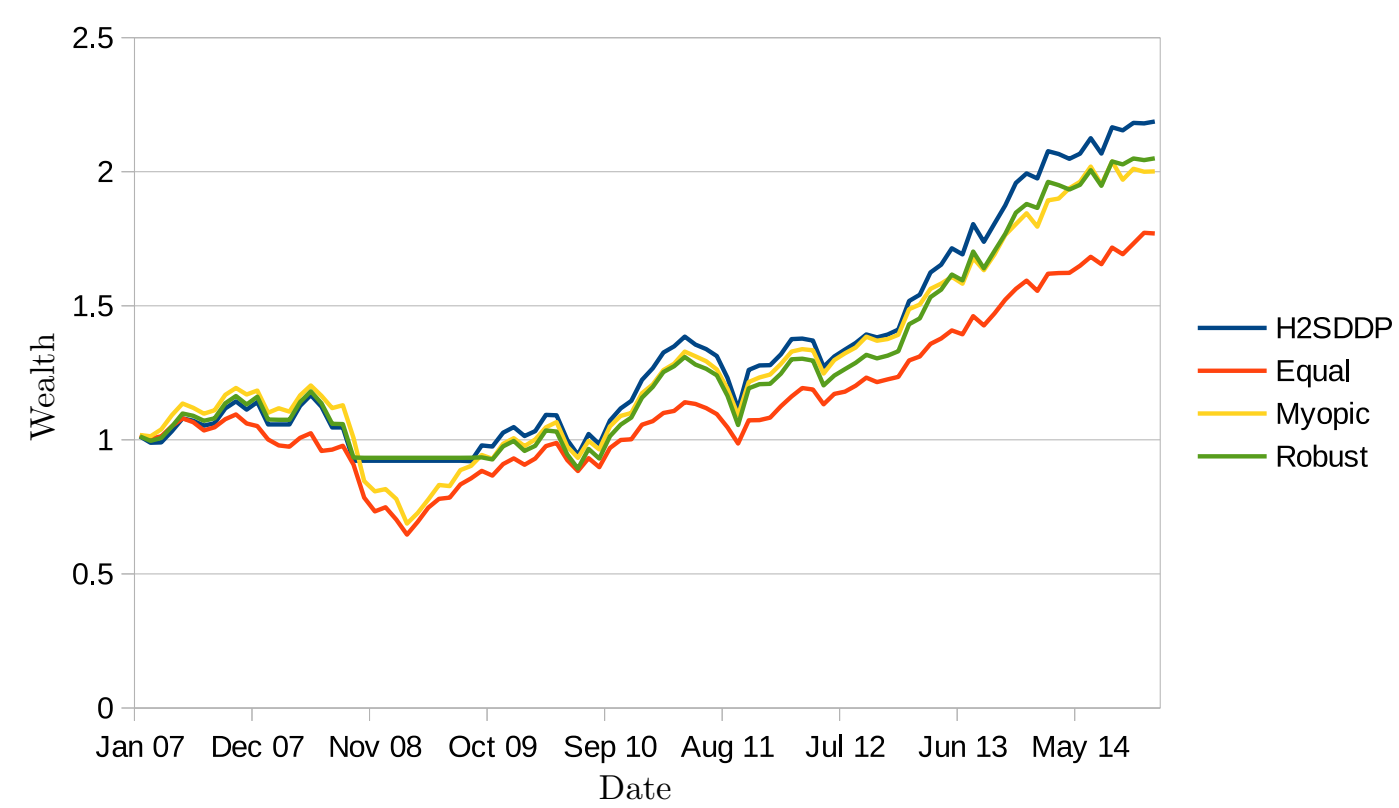

Figure 4.25: Comparing the different methods for asset allocation data form 2007 to 2014

The H2SDDP has almost 20\% and 50\% more income than the Myopic and equal policies, respectively. Analyzing more carefully the Figure 4.25, we can see that as soon as the H2SDDP observe the downward trend it allocates everything on the risk-free asset. However, there is a delay to see the upward trend of the market.

Figure 4.26 compares the state of the market with the performance of the H2SDDP. With it, becomes clear that the model can react to changes in the market behavior, even in a downtrend, this show how important is to have a constant evaluation of the portfolio. Considering the choices on Figure 4.25 the best investment for this period is H2SDDP. In this scenario Robust model perform very similar to the H2SDDP, to estimate property the interval require further investigation. 


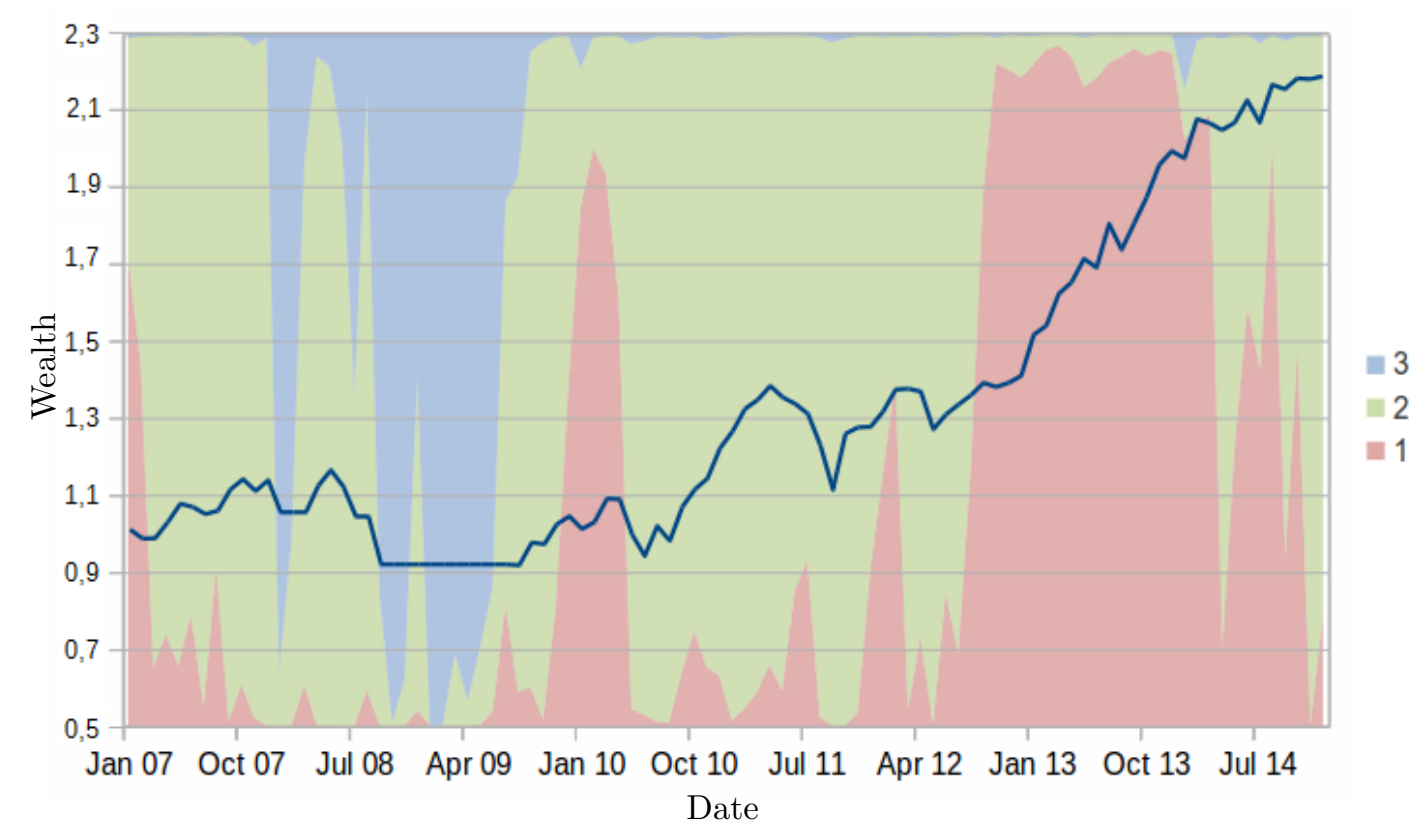

Figure 4.26: Comparing the market states with the performance of the H2SDDP for data form 2007 to 2014

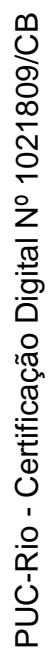

Moreover, to better judge the performance of the models over different periods, we simulate the investment for 6 consecutively months in each strategy as if it was a fund. Figure 4.27 presents the semiannual cumulative performance for every month.

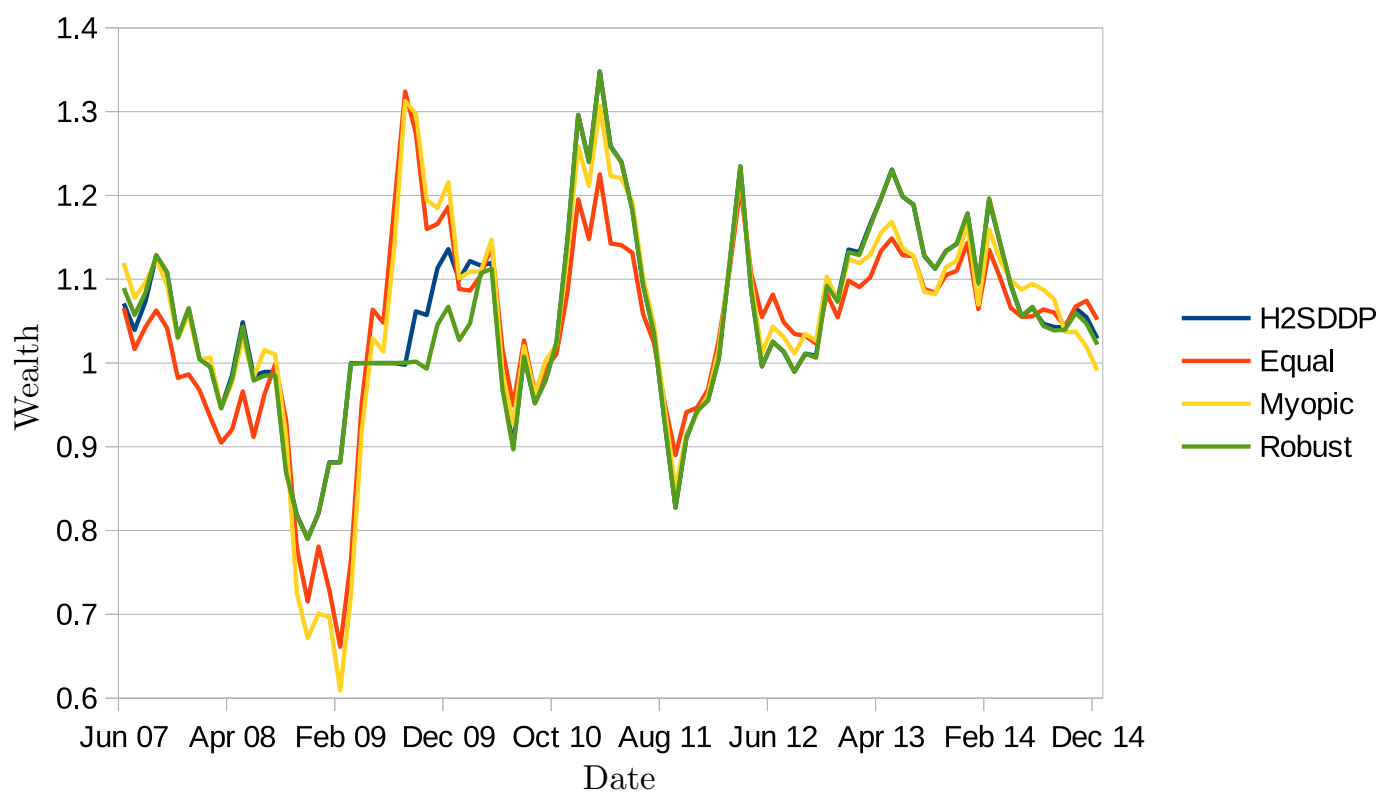

Figure 4.27: Trailing returns for asset allocation methods with monthly data form 2007 to 2014

Using short sell the model can take advantage of these situations, and without it the only reasonable action is to invest on risk-free asset. Therefore, 
we want to verify if the use of short selling can help the model improve the policy return. In order to test how H2SDDP will perform with short sell, we implement a model that allow short selling but limit it on the total wealth of the portfolio in the last period. On Figure 4.28 we compare the performance of H2SDDP, allowing short selling, with other methods for the same period.

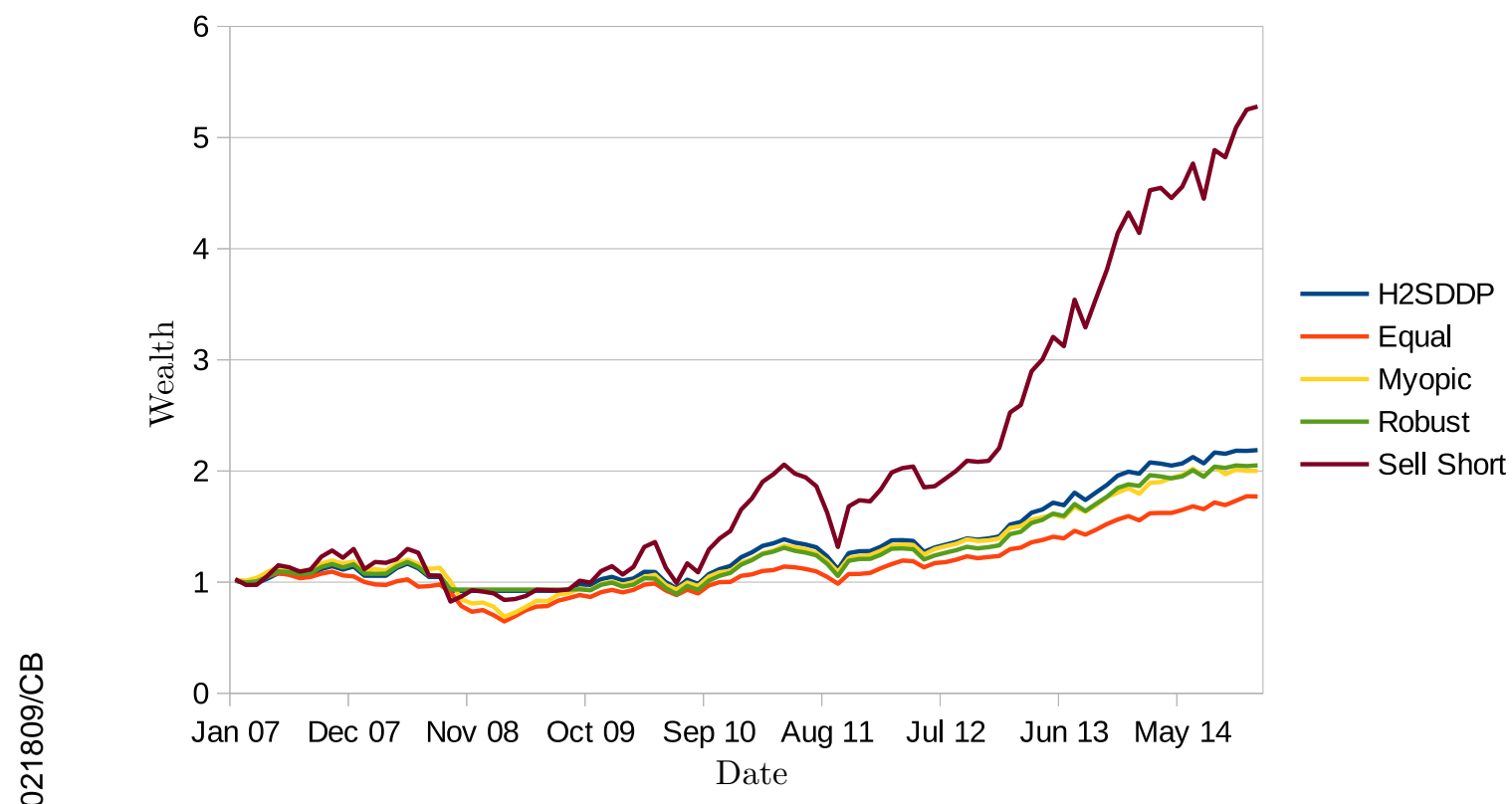

Figure 4.28: Comparing the performance of H2SDDP with sell short sell short with other methods, monthly data form 2007 to 2014

The sell short model has by far the highest cumulative return of the models and also perform similar to the H2SDDP, without short sell, in the downward trend, investing most of the portfolio on the risk free asset. However, the sell short took longer to identify the downward trend it also react very fast when the trend changed.

It seems that allowing short sell makes the model enhance the high returns, short selling the more stable assets to invest more on asset with high expected returns. The final cumulative return with sell short(527\%) was more than twice the value of the second best model H2SDDP (218\%).

In the trailing returns on Figure 4.29 we can observe that Sell Short H2SDDP has the higher return for most of the period, but this is also a more risk model than the H2SDDP without short selling. Although, the model has it lowest' possible return at the end of the year 2011 the Myopic and Equal models reaches lower returns than this, in the begin of 2009. Is is also possible to decrease the $\lambda$, that was 0.1 , to obtain more stable performance. 


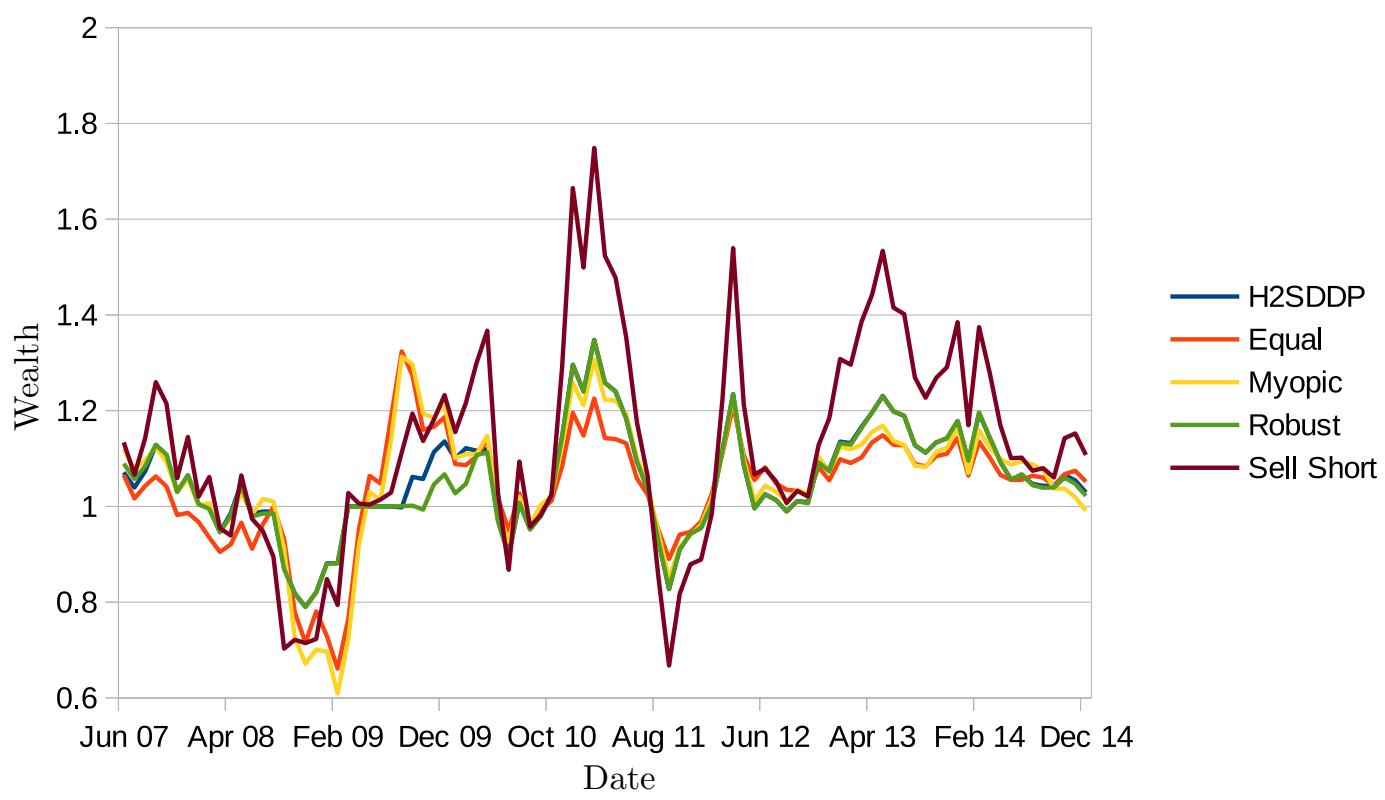

Figure 4.29: Trailing returns of H2SDDP with sell short sell and other methods, monthly data form 2007 to 2014

\section{Experiment with Daily Data Set}

To be able to observe how the model will behavior with more assets and more volatility, we perform an experiment with daily returns from 10 industrial portfolios. It was used data from 2007 to 2014, it not possible to create cuts for 2012 days, thus the daily data set was divide in intervals of 22 days, so the optimization has 22 cuts and every 22 day the model will be retrained. The only difference in the parameters was the $\lambda=0.03$.

Figure 4.30 shows the results for the H2SSP and, Equal weight and Myopic, the figure emphasizes the importance of a dynamic allocation, in which the model can change allocation when facing critical events, without it the portfolio depends on the tendency of the market to get good results. By the end of 2008 the cumulative return for the period was $113.16 \%, 71.16 \%$ and $73.98 \%$, for the H2SSP, Equal weight and Myopic, respectively. Figure 4.31 is trailing returns for this experiment. 


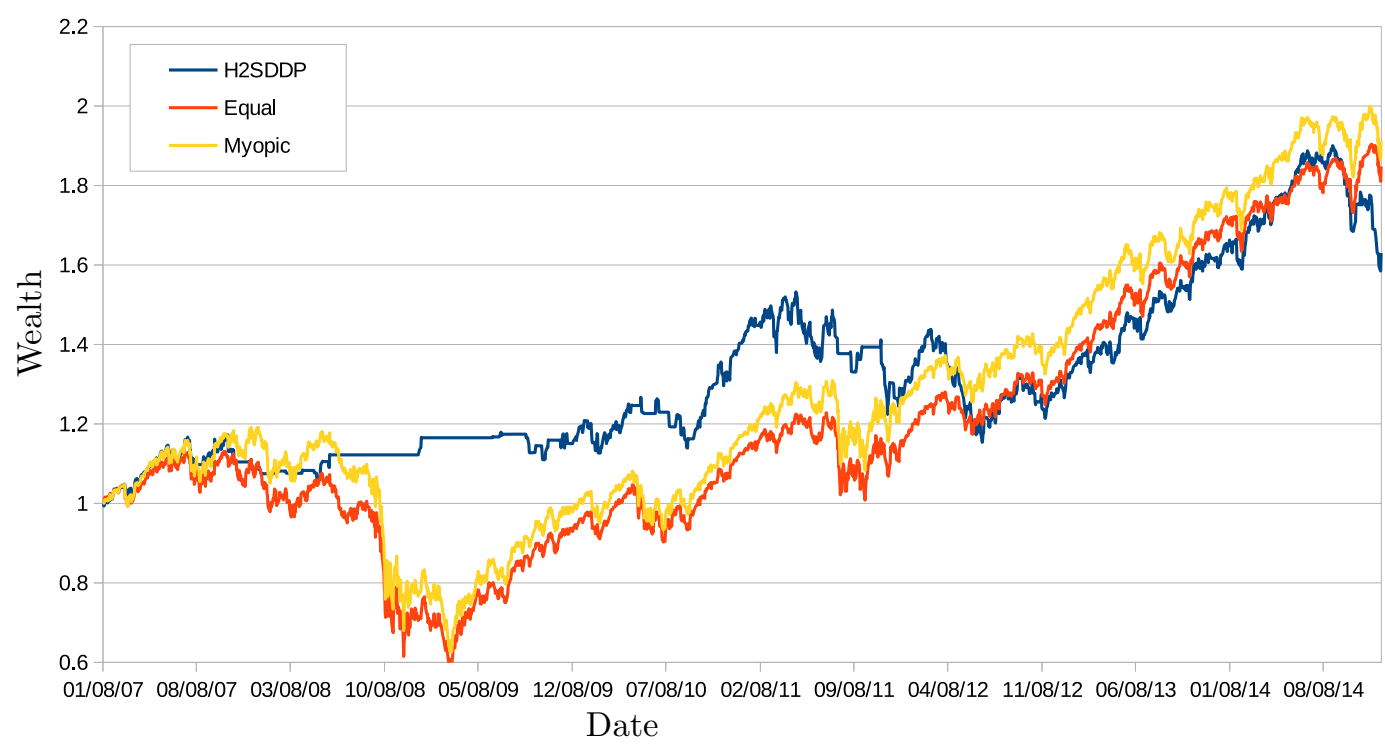

Figure 4.30: Cumulative performance for asset allocation methods with daily data set form 2007 to 2014

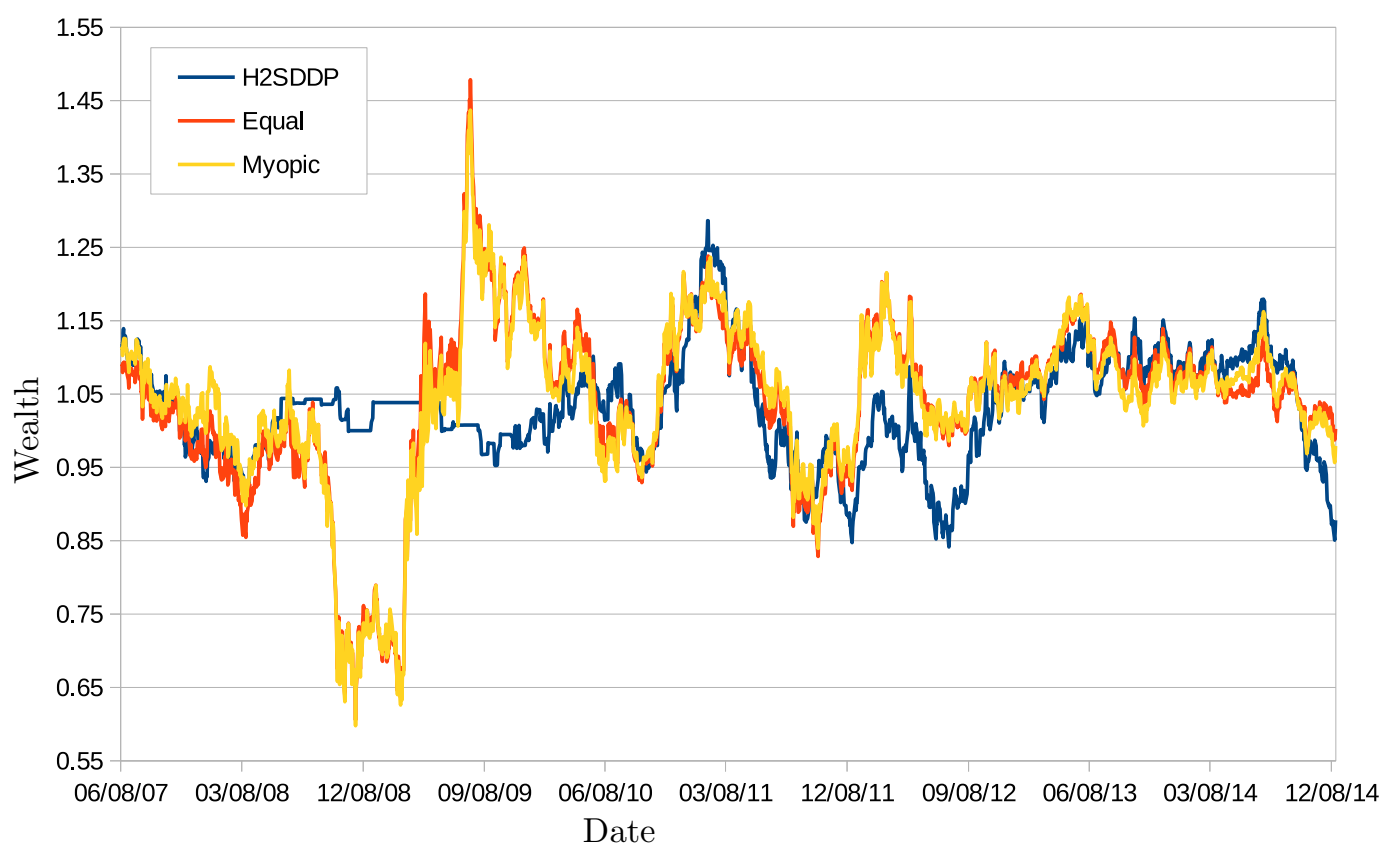

Figure 4.31: Trailing returns for asset allocation methods with daily data set form 2007 to 2014

Although, H2SDDP strategy fails to predict the upward tendency after 2008, it has clearly a more controlled risk than the other strategies; it has lower CVaR and standard deviation for this period (2008 to 2014). This can be evidence that the model should be retrained more frequently than 22 days. Besides that, some further investigation is necessary to better compare these strategies using different data sets with more diversity. 


\section{5 \\ Conclusions and Future Works}

This thesis aims at contributing for the optimization of models for asset allocation under uncertainty. Different techniques are proposed herein to assist in the asset allocation problem. Experiments were performed to evaluate the behavior of ours approaches and they turn down to be very promising for both of the presented cases.

It is a challenge to find the portfolio that best suit the investor's needs. The uncertainty surrounding future returns and the behavior of the assets is a delicate issue in its own, but when the irrationality caused by human emotions is added to the equation, the problem becomes even more difficult.

In the traditional asset allocation model, the investor has to choose the return or the risk of the portfolio. Such mean-variance model has several issues, however and, as a consequence, we have some proposals for improving the mean-variance model by making it less sensitive to such issues. One of these proposals is the Black-Litterman, which combines the investor's vision with the mean-variance model and the CAPM theory.

Although it is undeniable that the Black-Litterman technique is able to mitigate most of the shortcomings of the traditional method proposed by Markowitz, the construction of views can be a confusing process and depends largely on the investor's ability to quantify something extremely subjective.

We propose a new way to solve this problem by using questionnaires and the ZAPROS-III method to construct the views. Techniques like the VDA enable us to transform the answers provided in the sets of questions into views of the Black-Litterman model. A case study based on Brazilian stocks was conducted as a demonstration of how to use the methodology, and the results were as expected.

The most important advantage of the proposed solution is that it allows an investor to create his own portfolio, based on his own opinion, and without the help of an expert. The final allocation will be optimized considering the investor vision about the future, this typically will create a more diversified and less risk portfolio. It also makes it easier for the investor to manifest his own opinion and facilitates the process, allowing the investor to modify his portfolio more frequently. 
While we believe that this approach presents a lot of advantages, it also has its limitations. The ZAPROS method is not adequate for a larger number of alternatives or criteria, rendering this method applicable only to cases involving a few stocks and questions. This shortcoming is a relative one, as the focus of this proposal has always been to create a simple method, we believe it is not reasonable to ask an investor too many questions. Besides, even though only two views were constructed in the scope of this work, a larger number of views may be constructed in order to represent other visions.

It would be interesting to have further work done in the sense of proposing or developing new ways to improve the acquisition of the necessary parameters for the Black-Litterman views, like the expected return. Future work could also put other risk functions to the test and analyze the model's behavior. Also the construction of the views can be done in multilevel using ORCLASS, or some other similar method, to select the assets of each view and then use ZAPROS to determine the weight of the assets in the views.

Aiming at a multistage solution that also analyzes the assets in a more qualitative way, this work proposes the use of stochastic programming for assets allocation. Most of the proposed solutions in the literature cannot handle a large number of stages, but the SDDP method has been efficiently used for large-scale problems for several years now, and was thus chosen in this work for the multistage asset allocation.

Largely used for hydrothermal scheduling problems, SDDP is specially suited for large-scale problems. In it, the algorithm implicitly represent the scenarios tree, building at each iteration better approximations for the worth function for each stage. The time independence hypothesis of the original proposed SDDP makes it non-applicable to some types of problems. While some proposals to adapt the SDDP to consider the time dependence already exist, none of these can be directly applied to the asset allocation problem. Hence the methodology in this work, which models this dependency by using hidden Markovian states. In addition, as there is some ambiguity in the joint probability of the returns' distribution, a more general method with ambiguity aversion has also been developed.

In the experiments section we present the metrics for the data sets, comparison between Monte Carlo and Latin Hypercube Sampling, the sensitivity analyses of the model, showing how H2SDDP performs with different parameters, and the final simulations of the proposed methods with out of sample data. Besides showing how the optimization models would perform as an actual fund, the experiments emphasizes the importance continuous re-evaluation of the portfolio. 
As the use of multistage stochastic techniques for portfolio optimization becomes more and more widespread, both fields experience constant changes and new ideas and methods are often proposed. Therefore, several ideas for possible future work arose during the development of this thesis. One of them would be to constraint the CVaR, aiming at maximizing the expected value of the portfolio. The advantage in this case would be to have an easy way to calculate the lower bound, as well as an easier way of interpreting the model's characteristics, risk constraint and objective function. Also, choosing a maximum value for the CVaR makes interpretation easier and is a more natural choice than the risk aversion index.

The multistage CVaR constraint has been suggested for the hydrothermal scheduling problem [90], but adding this constraint to such model my render it unfeasible. Thus, the normal approach for such cases is to use Lagrangian relaxation to penalize violations, of the constraint, in the objective function. In asset allocation problems, however, such feasibility issue is not expected because allocating everything in the risk free asset is always feasible (just has to have $\gamma$ bigger than the transactional cost). This makes the model significantly more straightforward and not imposing any changes to the objective function.

The main difference in this model is that the optimization needs to be done in two-stages. The variables $y_{t i}$ must be created, representing a vector with the amount invested in each asset in $t$. Adding these ideas with H2SDDP, we acquire

$$
\begin{aligned}
& \begin{array}{l}
Q_{t}\left(\mathbf{y}_{t} \mid k\right)= \\
\max _{\mathbf{x}_{t}, \mathbf{d}_{t}^{+}, \mathbf{d}_{t}^{-}, \boldsymbol{\delta}, z}-c \sum_{i=1}^{A}\left(d_{t i}^{+}+d_{t i}^{-}\right) \\
+\sum_{j=1}^{K} P(j \mid k) \sum_{s=1}^{N_{t}} P(s \mid j)\left\{\mathbf{r}_{t+1}^{\top}(s) \mathbf{x}_{t}+Q_{t+1}\left(\left(1+\mathbf{r}_{t+1}(s)\right)^{\top} \mathbf{x}_{t} \mid j\right)\right\} \\
\text { s. t. } \quad \sum_{i=1}^{A} x_{t i}+c \sum_{i=1}^{A}\left(d_{t i}^{+}+d_{t i}^{-}\right)=\sum_{i=1}^{A} y_{t i} \\
\quad x_{t i}-d_{t i}^{+}+d_{t i}^{-}=y_{t i} \quad \forall i \in \mathcal{A} \backslash\{1\} \\
\quad z+\frac{\sum_{j=1}^{K} \sum_{s=1}^{N_{t}} P(j \mid k) P(s \mid j) \delta_{j s}}{(1-\alpha)}+c \sum_{i=1}^{A}\left(d_{t i}^{+}+d_{t i}^{-}\right) \leq \gamma\left(\sum_{i=1}^{A} y_{t i}\right) \\
\quad \delta_{j s} \geq-z-\mathbf{r}_{t+1}^{\top}(s) \mathbf{x}_{t} \quad \forall j \in\{1, \ldots, K\}, s \in\left\{1, \ldots, N_{t}\right\} \\
\quad \boldsymbol{\delta}, \mathbf{x}_{t}, \mathbf{d}_{t}^{+}, \mathbf{d}_{t}^{-} \geq 0
\end{array}
\end{aligned}
$$

where $P(s \mid j)=\frac{1}{N_{t}}$. Further experimentation will be made with this model in near future, in order to compare it with the other models developed in this thesis.

The estimation of the joint distributions of the returns is especially 
problematic in asset allocation problems, but there are some approaches that may be used in order to minimize this issue. One of them is to incorporate robust approaches to the models proposed here by using the Bertsimas ideas on the probability states of the Markov model. Such proposal is an extension of the ambiguity aversion model presented in Section 4.3(d).

In addition, it must be said that some of the concepts proposed here could not be computed due to the long computational time needed to run the experiments, which is why it would be interesting to have some proposal to reduce the algorithms' execution time. In this regard, our research indicates that a first and straightforward possible solution would be to design parallel algorithms, making better use of modern computers that currently have multiple cores. Another alternative would be to create heuristics to remove some of the cutting planes' cuts, a solution that has already shown some improvements in other researches.

There is also the need to better understand the behavior of the HMM in practice, and how to better estimate the initial probabilities. The method kmeans is a suitable method for this situation, but may have unwanted collateral effects. We could not find a better alternative in literature, but it is possible to test with manual classification. 


\section{Bibliography}

[1] FABOZZI, F. J.; KOLM, P. N.; PACHAMANOVA, D.; FOCARDI, S. M.. Robust portfolio optimization and management. John Wiley \& Sons, 2007. 1, 2, 4.2(b)

[2] BERTSIMAS, D.; GUPTA, V.; PASCHALIDIS, I. C.. Inverse optimization: a new perspective on the black-litterman model. Operations research, 60(6):1389-1403, 2012. 1.4, 3.1

[3] MARKOWITZ, H.. Portfolio selection. The Journal of Finance, 7(1):77-91, Março 1952. 2, 2.2

[4] BLACK, F.; LITTERMAN, R.. Global Portfolio Optimization. Financial Analysts Journal, 48(5), 1992. 2.3, 3.1, 3.3

[5] MICHAUD, R. O.. The markowitz optimization enigma: is' optimized'optimal? Financial Analysts Journal, 45(1):31-42, 1989. 2.3

[6] TÜTÜNCÜ, R. H.; KOENIG, M.. Robust asset allocation. Annals of Operations Research, 132(1):157-187, 2004. 2.3

[7] BERA, A.; PARK, S.. Optimal portfolio diversification using the maximum entropy principle. Econometric Reviews, 27(4-6):484512, 2008. 2.3

[8] ERDOGAN, E.; GOLDFARB, D.; IYENGAR, G.. Robust portfolio management. Technical report, 2004. 2.3

[9] BEST, M. J.; GRAUER, R. R.. Sensitivity analysis for mean-variance portfolio problems. Management Science, 37(8):980-989, 1991. 2.3

[10] CHOPRA, V. K.; ZIEMBA, W. T.. The effect of errors in means, variances, and covariances on optimal portfolio choice. 1993. 2.3

[11] FROST, P. A.; SAVARINO, J. E.. For better performance: Constrain portfolio weights. 1988. 2.3

[12] JAGANNATHAN, R.; MA, T.. Risk reduction in large portfolios: Why imposing the wrong constraints helps. The Journal of Finance, 58(4):1651-1684, 2003. 2.3 
[13] BLACK, F.; LITTERMAN, R.. Asset Allocation: Combining Investor Views with Market Equilibrium. The Journal of Fixed Income, 1990. 3.1, 3.3, 3.3, 3.3(c)

[14] WALTERS, J.. The black-litterman model in detail. Available at SSRN 1314585, 2011. 3.1, 3.3(d), 3.4(b)

[15] SATCHELL, S.; SCOWCROFT, A.. A demystification of the BlackLitterman model: Managing quantitative and traditional portfolio construction. Journal of Asset Management, 1(2):138-150, Sept. 2000. 3.1

[16] HE, G.; LITTERMAN, R.. The intuition behind black-litterman model portfolios. Available at SSRN 334304, 2002. 3.1, 3.3(c)

[17] MANKERT, C.. The Black-Litterman Model : Towards its use in practice. PhD thesis, KTH, Management Control, 2010. QC 20101202. 3.1

[18] HEROLD, U.. Computing implied returns in a meaningful way. Journal of Asset Management, 6(1):53 - 64, June 2005. 3.1

[19] IDZOREK, T.. A step-by-step guide to the black-litterman model. Forecasting Expected Returns in the Financial Markets, p. 17, 2002. (document), 3.1, 3.2, 3.3(d)

[20] FERNANDES, B.; FERNANDES, C.; STREET, A.. An asset allocation model with inequalities constraints and coherent risk measure: an application to Brazilian equities. Revista de Finanças Aplicadas, p. $1-27,2013.3 .1$

[21] MEUCCl, A.. The Black-Litterman Approach: Original Model and Extensions. Social Science Research Network Working Paper Series, Apr. 2008. 3.1

[22] EVANGELOU, C. E.; KARACAPILIDIS, N. I.; KHALED, O. A.. Interweaving knowledge management, argumentation and decision making in a collaborative setting: the kad ontology model. International Journal of Knowledge and Learning, 1(1/2):130-145, 2005. 3.1

[23] CASTRO, A.; PINHEIRO, P.; PINHEIRO, M.. An approach for the neuropsychological diagnosis of alzheimer's disease: A hybrid model in decision making. In: Wen, P.; Li, Y.; Polkowski, L.; Yao, 
Y.; Tsumoto, S.; Wang, G., editors, ROUGH SETS AND KNOWLEDGE TECHNOLOGY, v. 5589 of Lecture Notes in Computer Science, p. 216-223. Springer Berlin / Heidelberg, 2009. 3.1

[24] TONCOVICH, A.; TURÓN, A.; ESCOBAR, M. T.; MORENO-JIMÉNEZ, J. M.. A quantitative approach to identify the arguments that support decisions in e-cognocracy. IJKSR, 2(3):36-48, 2011. 3.1

[25] PINHEIRO, P.; SOUZA, G.; CASTRO, A.. Structuring of the multicriteria problem for production of newspaper. Operational research. Rio de Janeiro, 28(2):203-216, 2008. 3.1

[26] MENDES, M.; CARVAlHO, A. L.; FURTADO, E.; PINHEIRO, P. R.. A co-evolutionary interaction design of digital tv applications based on verbal decision analysis of user experiences. In: WSKS (2), p. 702-711, 2008. 3.1

[27] TAMANINI, I.; CARVALHO, A. L.; CASTRO, A. K. A.; PINHEIRO, P. R. A novel multicriteria model applied to cashew chestnut industrialization process. v. 58 of Advances in Intelligent and Soft Computing, p. 243-252. Springer Berlin Heidelberg, 2009. 3.1

[28] TAMANINI, I.; CASTRO, A. K. A.; PINHEIRO, P. R.; PINHEIRO, M. C. D.. Verbal decision analysis applied on the optimization of alzheimer's disease diagnosis: A case study based on neuroimaging. In: SOFTWARE TOOLS AND ALGORITHMS FOR BIOLOGICAL SYSTEMS, v. 696 of Advances in Experimental Medicine and Biology, p. 555-564. Springer New York, 2011. 3.1

[29] TAMANINI, I.; PINHEIRO, P. R.; PINHEIRO, M. C. D.. The choice of neuropathological questionnaires to diagnose the alzheimer's disease based on verbal decision analysis methods. In: INFORMATION COMPUTING AND APPLICATIONS, v. 6377 of Lecture Notes in Computer Science, p. 549-556. Springer Berlin Heidelberg, 2010. 3.1

[30] CASTRO, A. K. A. D.; PINHEIRO, P. R.; DANTAS PINHEIRO, M. C.; TAMANINI, I.. Towards the applied hybrid model in decision making: A neuropsychological diagnosis of alzheimer's disease study case. International Journal of Computational Intelligence Systems, 4(1):89-99, 2011. 3.1 
[31] TAMANINI, I.. Improving the ZAPROS method considering the incomparability cases. Master's thesis, University of Fortaleza, Applied Informatics, Ceará, Brazil, 2010. 3.1, 3.2(b), 3.2(b), 3.4(b)

[32] MACHADO, T.; MENEZES, A.; TAMANINI, I.; PINHEIRO, P.. A hybrid model in the selection of prototypes for educational tools: An applicability in verbal decision analysis. In: SYMPOSIUM ON COMPUTATIONAL INTELLIGENCE IN MULTICRITERIA DECISIONMAKING, p. 135-142. IEEE, 2011. 3.2

[33] LARICHEV, O. I.. Ranking multicriteria alternatives: The method ZAPROS III. European Journal of Operational Research, 131(3):550-558, June 2001. 3.2, 3.2(b)

[34] LARICHEV, O.; MOSHKOVICH, H.. Verbal Decision Analysis for Unstructured Problems. Theory and decision library: Series C, Game theory, mathematical programming, and operations research. Springer, 1997. 3.2, 3.2(a)

[35] GOMES, L.; MOSHKOVICH, H.; TORRES, A.. Marketing decisions in small businesses: how verbal decision analysis can help. International Journal of Management and Decision Making, 11(1):19-36, 2010. 3.2

[36] CHRISSIS, M.; KONRAD, M.; SHRUM, S.. Cmmi: Guidelines for Process Integration and Product Improvement. Series in Software Engineering. Prentice Hall, second edition, 2007. 3.2, 3.2(b), 3.2(b)

[37] USTINOVICH, L.; KOCHIN, D.. Verbal decision analysis methods for determining the efficiency of investments in construction. Foundations of Civil and Environmental Engineering, 5(1):35-46, 2004. 3.2, 3.2(b)

[38] BRASIL, A.. A novel approach based on multiple criteria decision aiding methods to cope with classification problems. Master's thesis, University of Fortaleza, Applied Informatics, Ceará, Brazil, 2010. 3.2

[39] BRASIL, A.; PINHEIRO, P. R.; COELHO, A. L. V.. The impact of the prototype selection on a multicriteria decision aid classification algorithm. In: Sobh, T., editor, INNOVATIONS AND ADVANCES IN COMPUTER SCIENCES AND ENGINEERING, p. 379-382. Springer Netherlands, 2010. 3.2 
[40] BRASIL, A.; PINHEIRO, P. R.; COELHO, A. L. V.. Towards the Early Diagnosis of Alzheimer's Disease through the Application of a Multicriteria Classification Model, v. 1. Rijeka: Intech - Open Access Publisher, 2012. 3.2

[41] MANKERT, C.. The Black-Litterman Model: mathematical and behavioral finance approaches towards its use in practice. PhD thesis, $\mathrm{KTH}$, 2006. 3.3

[42] ELTON, E. J.; GRUBER, M. J.; BROWN, S. J.; GOETZMANN, W. N.. Modern portfolio theory and investment analysis. John Wiley \& Sons, 2009. 3.3(a), 4.1

[43] SHARPE, W. F.. Capital asset prices: A theory of market equilibrium under conditions of risk*. The journal of finance, 19(3):425442, 1964. 3.3(a)

[44] WALTERS, J.. The factor tau in the black-litterman model. Available at SSRN 1701467, 2010. 3.3(a), 3.3(d)

[45] THEIL, H.. Principles of econometrics. 1971. 3.3(c)

[46] HE, G.; LITTERMAN, R.. The intuition behind black-litterman model portfolios. Goldman Sachs Asset Management Working paper, 1999. 3.3(d)

[47] SILVA, A. S.; LEE, W.; PORNROJNANGKOOL, B.. The blacklitterman model for active portfolio management. The Journal of Portfolio Management, 35(2):61-70, 2009. 3.4(b)

[48] PEREIRA, M. V. F.; PINTO, L. M. V. G.. Multi-stage stochastic optimization applied to energy planning. Math. Program., 52(2):359375, Oct. 1991. 4.1, 4.2, 4.3(c)

[49] DONOHUE, C. J.; BIRGE, J. R.. The abridged nested decomposition method for multistage stochastic linear programs with relatively complete recourse. Algorithmic Operations Research, 1(1), 2006. $4.1,4.2$

[50] CHEN, Z.-L.; POWELL, W. B.. Convergent cutting-plane and partial-sampling algorithm for multistage stochastic linear programs with recourse. Journal of Optimization Theory and Applications, 102(3):497-524, 1999. 4.1, 4.2 
[51] SHAPIRO, A.; TEKAYA, W.; DA COSTA, J. P.; SOARES, M. P.. Risk neutral and risk averse stochastic dual dynamic programming method. European journal of operational research, 224(2):375-391, 2013. 4.1

[52] INFANGER, G.; MORTON, D. P.. Cut sharing for multistage stochastic linear programs with interstage dependency. Mathematical Programming, 75(2):241-256, 1996. 4.1

[53] PEREIRA, M.; PINTO, L.. Stochastic optimization of a multireservoir hydroelectric system: a decomposition approach. Water resources research, 21(6):779-792, 1985. 4.1, 4.2(c)

[54] MO, B.; GJELSVIK, A.; GRUNDT, A.. Integrated risk management of hydro power scheduling and contract management. Power Systems, IEEE Transactions on, 16(2):216-221, 2001. 4.1, 4.3(c)

[55] PHILPOTT, A. B.; DE MATOS, V. L.. Dynamic sampling algorithms for multi-stage stochastic programs with risk aversion. European Journal of Operational Research, 218(2):470-483, 2012. 4.1, 4.3(c)

[56] CONT, R.. Empirical properties of asset returns: stylized facts and statistical issues. 2001. 4.1

[57] MORETTIN, P. A.; TOLOI, C.. Análise de séries temporais. Blucher, 2006. 4.1

[58] VALLADÃO, D. M.; VEIGA, Á.; VEIGA, G.. A multistage linear stochastic programming model for optimal corporate debt management. European Journal of Operational Research, 237(1):303$311,2014.4 .1$

[59] DANTZIG, G. B.; INFANGER, G.. Multi-stage stochastic linear programs for portfolio optimization. Annals of Operations Research, 45(1):59-76, 1993. 4.1

[60] SHAPIRO, A.; DENTCHEVA, D.; RUSZCZYŃSKI, A.. Lectures on stochastic programming: modeling and theory, v. 16. SIAM, 2014. 4.1, 4.2(a), 4.2(d), 4.3(a)

[61] ROCKAFELLAR, R. T.; URYASEV, S.. Conditional value-at-risk for general loss distributions. Journal of Banking and Finance, 26(7):1443-1471, July 2002. 4.1, 4.2(b), 4.3(b) 
[62] ROCKAFELLAR, R. T.; URYASEV, S.. Optimization of conditional value-at-risk. Journal of Risk, 2:21-41, 2000. 4.1, 4.2(b)

[63] SHAPIRO, A.. Analysis of stochastic dual dynamic programming method. European Journal of Operational Research, 209(1):63-72, 2011. 4.1, 4.2(b), 1, 4.2(b), 4.2(c)

[64] BENDERS, J.. Partitioning procedures for solving mixedvariables programming problems. Numerische Mathematik, $4(1): 238-252,1962.4 .2$

[65] ARTZNER, P.; DELBAEN, F.; EBER, J.-M.; HEATH, D.. Coherent measures of risk. Mathematical finance, 9(3):203-228, 1999. 4.2(b), 4.2(b), 2, 4.3(b)

[66] GROOTVELD, H.; HALLERBACH, W.. Variance vs downside risk: Is there really that much difference? European Journal of operational research, 114(2):304-319, 1999. 4.2(b)

[67] KROKHMAL, P.; PALMQUIST, J.; URYASEV, S.. Portfolio optimization with conditional value-at-risk objective and constraints. JOURNAL OF RISK, 4:11-27, 2002. 4.2(b)

[68] RUDlOFF, B.; STREeT, A.; VAlLADÃO, D. M.. Time consistency and risk averse dynamic decision models: Definition, interpretation and practical consequences. European Journal of Operational Research, 234(3):743-750, 2014. 4.2(b), 4.3(b)

[69] LINOWSKY, K.; PHILPOTT, A. B.. On the convergence of samplingbased decomposition algorithms for multistage stochastic programs. Journal of optimization theory and applications, 125(2):349366, 2005. 4.2(c)

[70] SHAPIRO, A.; TEKAYA, W.. Report for technical cooperation between georgia institute of technology and ons-operador nacional do sistema eletrico-risk averse approach. Technical report, Citeseer, 2011. 4.2(c)

[71] KOZMÍK, V.; MORTON, D. P.. Evaluating policies in risk-averse multi-stage stochastic programming. Mathematical Programming, p. 1-26, 2014. 4.2(c) 
[72] HINDSBERGER, M.; PHILPOTT, A.. Stopping criteria in sampling strategies for multistage slp-problems. In: CONFERENCE "APPLIED MATHEMATICAL PROGRAMMING AND MODELLING", VARENNA, ITALY, 2002. 4.2(c)

[73] RUBINSTEIN, R. Y.; KROESE, D. P.. Simulation and the Monte Carlo method, v. 707. John Wiley \& Sons, 2011. 4.2(d)

[74] MCKAY, M. D.; BECKMAN, R. J.; CONOVER, W. J.. Comparison of three methods for selecting values of input variables in the analysis of output from a computer code. Technometrics, 21(2):239-245, 1979. 4.2(d)

[75] KIM, S.-J.; KOH, K.; LUSTIG, M.; BOYD, S.; GORINEVSKY, D.. An interior-point method for large-scale 11-regularized least squares. Selected Topics in Signal Processing, IEEE Journal of, 1(4):606-617, Dec 2007. 4.3(b)

[76] HARTE, D.; PICKUP, M.; THOMSON, P.. Hidden markov models for new zealand hydro catchment inflows: a preliminary analysis. New Zealand Electricity Commission, 2007. 4.3(c)

[77] BONNANS, J. F.; CEN, Z.; CHRISTEL, T.. Energy contracts management by stochastic programming techniques. Annals of Operations Research, 200(1):199-222, 2012. 4.3(c)

[78] FAMA, E. F.. The behaviour of stock market prices. Journal of Business, p. 34-105, 1965. 4.3(c)

[79] GRANGER, C. W.; DING, Z.. Stylized facts on the temporal and distributional properties of daily data from speculative markets. UCSD Department of Economics Discussion Paper, p. 94-19, 1994. 4.3(c)

[80] RYDÉN, T.; TERÄSVIRTA, T.; ÅSBRINK, S.. Stylized facts of daily return series and the hidden markov model. Journal of applied econometrics, 13(3):217-244, 1998. 4.3(c)

[81] HASSAN, M. R.; NATH, B.. Stock market forecasting using hidden markov model: a new approach. In: INTELLIGENT SYSTEMS DESIGN AND APPLICATIONS, 2005. ISDA'05. PROCEEDINGS. 5TH INTERNATIONAL CONFERENCE ON, p. 192-196. IEEE, 2005. 4.3(c) 
[82] ELLIOTT, R. J.; SIU, T. K.. Strategic asset allocation under a fractional hidden markov model. Methodology and Computing in Applied Probability, 16(3):609-626, 2014. 4.3(c)

[83] ELLIOTT, R. J.; VAN DER HOEK, J.. An application of hidden markov models to asset allocation problems. Finance and Stochastics, 1(3):229-238, 1997. 4.3(c)

[84] BISHOP, C. M.; OTHERS. Pattern recognition and machine learning, v. 4. springer New York, 2006. 4.3(c)

[85] MACCHERONI, F.; MARINACCI, M.; RUFFINO, D.. Alpha as ambiguity: Robust mean-variance portfolio analysis. Econometrica, 81(3):1075-1113, 2013. 4.3(d)

[86] GARLAPPI, L.; UPPAL, R.; WANG, T.. Portfolio selection with parameter and model uncertainty: A multi-prior approach. Review of Financial Studies, 20(1):41-81, 2007. 4.3(d)

[87] CURTIN, R. R.; CLINE, J. R.; SLAGLE, N. P.; MARCH, W. B.; RAM, P.; MEHTA, N. A.; GRAY, A. G.. MLPACK: A scalable $\mathrm{C}++$ machine learning library. Journal of Machine Learning Research, 14:801-805, 2013. 4.4

[88] SANDERSON, C.; OTHERS. Armadillo: An open source c++ linear algebra library for fast prototyping and computationally intensive experiments. Report Version, 2, 2010. 4.4

[89] DEMIGUEL, V.; GARLAPPI, L.; UPPAL, R.. Optimal versus naive diversification: How inefficient is the $1 / \mathrm{n}$ portfolio strategy? Review of Financial Studies, 22(5):1915-1953, 2009. 4.4(d)

[90] COSTA JÚNIOR, L. C.. Representing CVaR Risk Aversion Constraint on Stochastic Dual Dynamic Programming with Application in the Hydrothermal Operation Planning. PhD thesis, Federal University of Rio de Janeiro, COPPE, 2013. 5 


\section{A \\ Appendix}

\section{A.1 Questionnaires}

The questions that were mention on section 3.4 are presented in Table A.1 and Table A.2. This first survey on Table A.1 is specific about companies and it is used to construct the assets view. Six questions about the companies covering issues such as risk, stability, innovation and profitability.

\begin{tabular}{|c|c|}
\hline Criteria & Possible values \\
\hline A. Risk & $\begin{array}{l}\text { A1. Low risk } \\
\text { A2. Medium risk } \\
\text { A3. High risk }\end{array}$ \\
\hline B. Stability & $\begin{array}{l}\text { B1. Company with years of market experience } \\
\text { and tradition } \\
\text { B2. Company with some market time } \\
\text { B3. Company with little market time }\end{array}$ \\
\hline C. Expected growth & $\begin{array}{l}\text { C1. Company with promising future and ac- } \\
\text { celerated growth } \\
\text { C2. Company that is expected some growth } \\
\text { C3. Company which is not expected growth }\end{array}$ \\
\hline D. Innovation & $\begin{array}{l}\text { D1. The company invests heavily in R\&D and } \\
\text { always comes up with new ideas } \\
\text { D2. The company invests little in } \\
\text { R\&D and new ideas usually arise } \\
\text { D3. The company does not invest in R\&D and } \\
\text { almost never comes up new ideas }\end{array}$ \\
\hline E. Profitability & $\begin{array}{l}\text { E1. Company always transfers profits to } \\
\text { shareholders } \\
\text { E2. Company usually transfers profits to } \\
\text { shareholders } \\
\text { E3. Company almost never transfers profits to } \\
\text { shareholders }\end{array}$ \\
\hline F. Employees & $\begin{array}{l}\text { F1. Highly qualified employees that are always } \\
\text { motivated } \\
\text { F2. Good employees that are usually motiv- } \\
\text { ated } \\
\text { F3. Employees without much qualification and } \\
\text { lack of motivation }\end{array}$ \\
\hline
\end{tabular}

Table A.1: Questionnaire about the stocks 
Second survey on sectors Table A.2 is more simple and relative to the sectors of the market.

\begin{tabular}{|l|l|}
\hline Criteria & Possible values \\
\hline A. Internal market & $\begin{array}{l}\text { A1. Very favorable to industry } \\
\text { A2. Favorable to industry } \\
\text { A3. Not favorable to industry }\end{array}$ \\
\hline B. External market & $\begin{array}{l}\text { B1. Very favorable to industry } \\
\text { B2. Favorable to industry } \\
\text { B3. Not favorable to industry }\end{array}$ \\
\hline C. Expected growth & $\begin{array}{l}\text { C1. It is expected a high growth } \\
\text { C2. It is expected some growth } \\
\text { C3. It is expected little or none growth }\end{array}$ \\
\hline
\end{tabular}

Table A.2: Questionnaire about the sectors

\section{A.2 Myopic prove}

Proposition 1 For the portfolio selection with no transaction cost and without temporal dependence the myopic policy is optimal, i.e. $Q_{t}(\cdot)$ is positive homogeneous. As a result, in this case one only needs to consider the return of the next moment to decide on an investment.

Proof: To prove by induction we will first show for the base case that $Q_{T-1}(\cdot)$, then assuming that the proposition holds up to $Q_{t+1}(\cdot)$ and deduce $Q_{t}(\cdot)$

For the base case the optimal value on $T$ depends on $W_{T-1}$ and $\overline{\mathbf{r}}_{[T-1]}$, it will be used $\mathbf{R}_{T}=\mathbb{1}+\mathbf{r}_{T}$, thus we have

$$
\begin{aligned}
Q_{T-1}\left(W_{T-1}, \overline{\mathbf{r}}_{[T-1]}\right)=\max _{\mathbf{x}_{t-1}} & \psi_{T-1}\left[\mathbf{R}_{T}^{\top} \mathbf{x}_{t-1} \mid \overline{\mathbf{r}}_{[T-1]}\right] \\
\text { s. t. } & \mathbb{1}^{\top} \mathbf{x}_{t-1}=W_{T-1} \\
& \mathbf{x}_{t-1} \geq 0
\end{aligned}
$$

For $t=T-2, \ldots, 0$

$$
\begin{aligned}
Q_{t}\left(W_{t}, \overline{\mathbf{r}}_{[t]}\right)=\max _{\mathbf{x}_{t}} & \psi_{t}\left[Q_{t+1}\left(W_{t}, \overline{\mathbf{r}}_{[t]}\right)\right] \\
\text { s. t. } & \mathbb{1}^{\top} \mathbf{x}_{t}=W_{t} \\
& \mathbf{r}_{t+1}\left(\overline{\mathbf{r}}_{[t]}\right)^{\top} \mathbf{x}_{t}=W_{t+1} \\
& \mathbf{x}_{t} \geq 0
\end{aligned}
$$


As $\mathbf{R}_{T}$ is independent from the past $\mathbf{r}_{[t-1]}$

$$
\begin{aligned}
Q_{T-1}\left(W_{T-1}\right)=\max _{\mathbf{x}_{t-1}} & \psi\left[\mathbf{R}_{T}^{\top} \mathbf{x}_{t-1}\right] \\
\text { s. t. } & \mathbb{1}^{\top} \mathbf{x}_{t-1}=W_{T-1} \\
& \mathbf{x}_{t-1} \geq 0
\end{aligned}
$$

For $t=T-2, \ldots, 0$

$$
\begin{aligned}
Q_{t}\left(W_{t}\right)=\max _{\mathbf{x}_{t}} & \psi\left[Q_{t+1}\left(W_{t+1}\right)\right] \\
\text { s. t. } & \mathbb{1}^{\top} \mathbf{x}_{t}=W_{t} \\
& \overline{\mathbf{R}}_{t+1}^{\top} \mathbf{x}_{t}=W_{t+1} \\
& \mathbf{x}_{t} \geq 0
\end{aligned}
$$

Let $\mathbf{y}_{t}=\frac{\mathbf{x}_{t}}{W_{t}}, \forall t=0, \ldots, T-1$

$$
\begin{aligned}
& Q_{T-1}\left(W_{T-1}\right)=\max _{\mathbf{y}_{T-1}} \psi\left[\mathbf{R}_{T}^{\top} \mathbf{y}_{T-1} W_{T-1}\right] \\
& \text { s. t. } \quad \mathbb{1}^{\top} \mathbf{y}_{T-1}=1 \\
& \mathbf{y}_{T-1} \geq 0 \\
& Q_{T-1}\left(W_{T-1}\right)=W_{T-1} \times \max _{\mathbf{y}_{T-1}} \psi\left[\mathbf{R}_{T}^{\top} \mathbf{y}_{T-1}\right] \\
& \text { s. t. } \quad \mathbb{1}^{\top} \mathbf{y}_{T-1}=1 \\
& \mathbf{y}_{T-1} \geq 0
\end{aligned}
$$

Inductive hypothesis, assuming that this proposition is valid for $t+1$

$$
Q_{t+1}\left(W_{t+1}\right)=W_{t+1} \times \prod_{t^{\prime}=t+1}^{T-1} Q_{t^{\prime}}(1)
$$

Inductive step, for $t$

$$
\begin{aligned}
Q_{t}\left(W_{t}\right)=\max _{\mathbf{x}_{t}} & \psi\left[Q_{t+1}\left(W_{t+1}\right)\right] \\
\text { s. t. } & \mathbb{1}^{\top} \mathbf{x}_{t}=W_{t} \\
& \mathbf{r}_{t+1}^{\top} \mathbf{x}_{t}=W_{t+1} \\
& \mathbf{x}_{t} \geq 0
\end{aligned}
$$


by our inductive hypothesis

$$
\begin{aligned}
& \psi\left[Q_{t+1}\left(W_{t+1}\right)\right]=\psi\left[W_{t+1} \times \prod_{t^{\prime}=t+1}^{T-1} Q_{t^{\prime}}(1)\right]=\psi\left[\mathbf{r}_{t+1}^{\top} \mathbf{x}_{t} \times \prod_{t^{\prime}=t+1}^{T-1} Q_{t^{\prime}}(1)\right] \\
& Q_{t}\left(W_{t}\right)=\prod_{t^{\prime}=t+1}^{T-1} Q_{t^{\prime}}(1) \times \max _{\mathbf{x}_{t}} \psi\left[\mathbf{r}_{t+1}^{\top} \mathbf{x}_{t}\right] \\
& \text { s. t. } \mathbb{1}^{\top} \mathbf{x}_{t}=W_{t} \\
& \mathbf{x}_{t} \geq 0
\end{aligned}
$$

Swapping $\mathbf{x}_{t}$ for $\mathbf{y}_{t} \times W_{t}$

$$
\begin{aligned}
& Q_{t}\left(W_{t}\right)=\prod_{t^{\prime}=t+1}^{T-1} Q_{t^{\prime}}(1) \times \max _{\mathbf{y}_{t}} \psi\left[\mathbf{r}_{t+1}^{\top} \mathbf{y}_{t} \times W_{t}\right] \\
& \text { s. t. } \mathbb{1}^{\top} \mathbf{y}_{t}=1 \\
& \mathbf{y}_{t} \geq 0
\end{aligned}
$$

$$
\begin{gathered}
Q_{t}\left(W_{t}\right)=W_{t} \times \prod_{t^{\prime}=t+1}^{T-1} Q_{t^{\prime}}(1) \times \max _{\mathbf{y} t} \quad \psi\left[\mathbf{r}_{t+1}^{\top} \mathbf{y}_{t}\right] \\
\text { s.t. } \quad \begin{array}{l}
\mathbb{1}^{\top} \mathbf{y}_{t}=1 \\
\mathbf{y}_{t} \geq 0
\end{array} \\
Q_{t}\left(W_{t}\right)=W_{t} \times Q_{t}(1) \times \prod_{t^{\prime}=t+1}^{T-1} Q_{t^{\prime}}(1) \\
\mathbf{Q}_{\mathbf{t}}\left(\mathbf{W}_{\mathbf{t}}\right)=\mathbf{W}_{\mathbf{t}} \times \prod_{\mathbf{t}^{\prime}=\mathbf{t}}^{\mathbf{T}-\mathbf{1}} \mathbf{Q}_{\mathbf{t}^{\prime}}(\mathbf{1})
\end{gathered}
$$

Note the first stage problem $t=1$ using formulation (A.1)

$$
\begin{aligned}
& Q_{0}\left(W_{0}\right)=\prod_{t^{\prime}=2}^{T-1} Q_{t^{\prime}}(1) \times \max _{\mathbf{x}_{1}} \quad \psi\left[\mathbf{R}_{2}^{\top} \mathbf{x}_{0}\right] \\
& \text { s. t. } \mathbb{1}^{\top} \mathbf{x}_{0}=W_{0} \\
& \mathbf{x}_{1} \geq 0
\end{aligned}
$$

As the first stage decisions $\mathbf{x}_{0}$ are independent from $Q_{t}(1) \forall t \in\{2, \ldots, T-1\}$, to retrieve optimal solution it is only necessary to solve the problem below ignoring future returns.

$$
\max _{\mathbf{x}_{1}} \psi\left[\mathbf{R}_{2}^{\top} \mathbf{x}_{1}\right]
$$




$$
\begin{array}{ll}
\text { s. t. } & \mathbb{1}^{\top} \mathbf{x}_{1}=W_{1} \\
& \mathbf{x}_{0} \geq 0
\end{array}
$$

Likewise, it is possible to obtain optimal solution for $Q_{t}\left(W_{t}\right) \forall t \in T$. 